What Influences how New Zealanders use Information Displays? An Analysis of Electronic Gaming Machine Records

By

Shannon Garland Duignan

\author{
A thesis \\ Submitted to the Victoria University of Wellington \\ In fulfilment of the requirements for the degree of \\ Master of Science in Cognitive and Behavioural Neuroscience
}

Victoria University of Wellington

2019 


\section{Table of Contents}

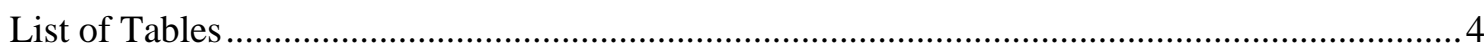

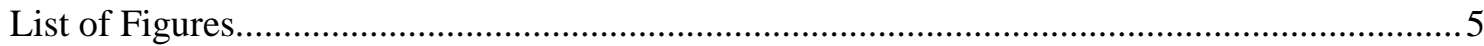

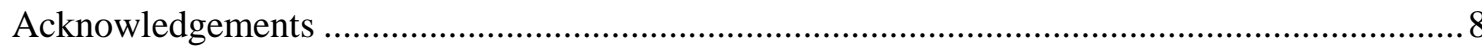



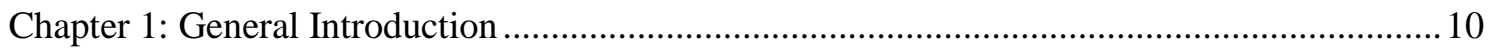

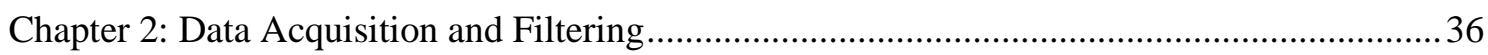

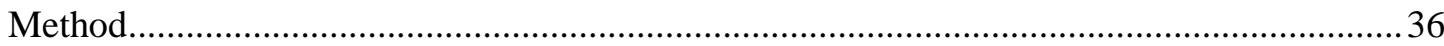

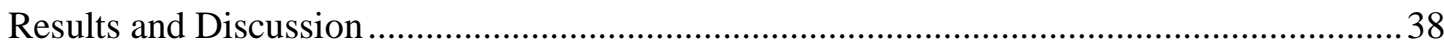

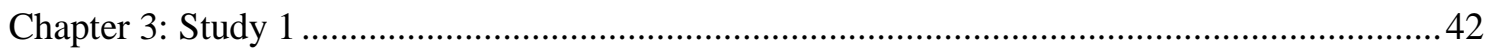

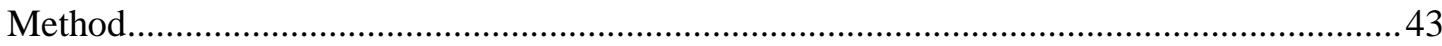



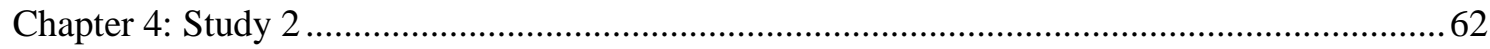

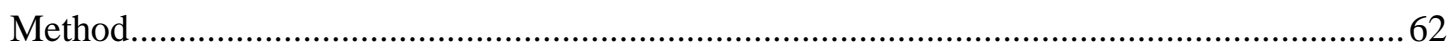

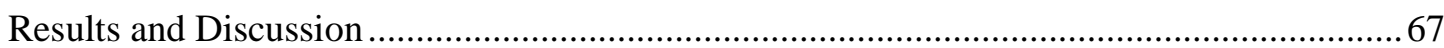

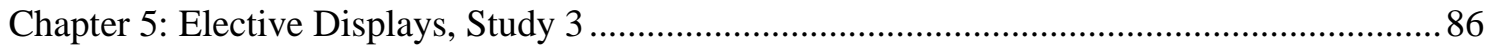

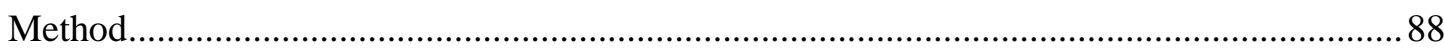

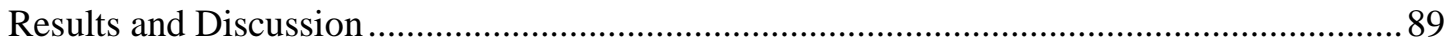

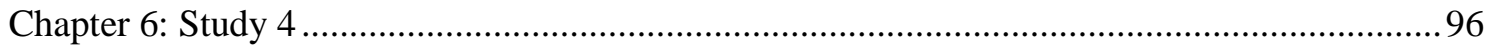

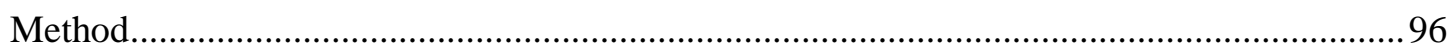

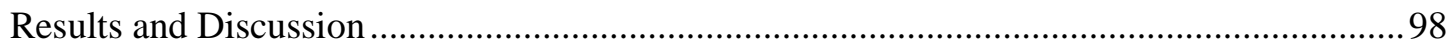

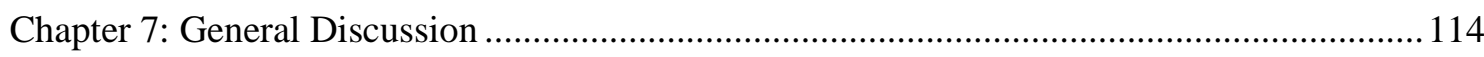

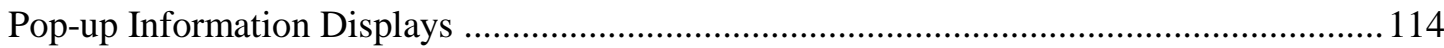




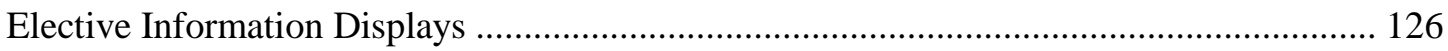

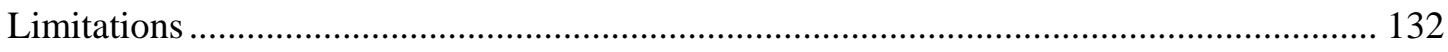

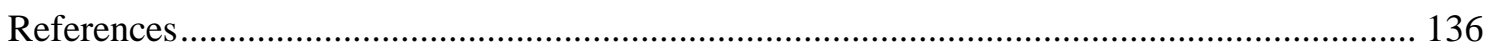

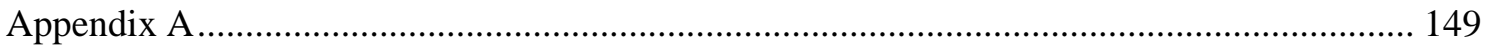

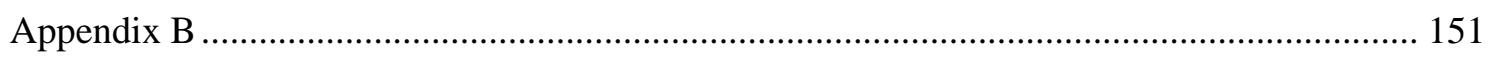




\section{List of Tables}

Table 3.1 Gambling session descriptive statistics .43

Table 3.2. Pop-up sessions by days of the week .48

Table 3.3 Pop-up sessions by manufacturers .50

Table 4.1. ANOVA results of final model type III sum of squares .68

Table 4.2 Confusion Matrix for training dataset .82

Table 4.3 Confusion Matrix for test dataset .83

Table 5.1. Elective information displays accessed by days of the week .91

Table 5.2 Matrix of significance values for pairwise comparisons across the day of the week .92

Table 5.3. Elective information displays accessed by manufacturers .93

Table 5.4 Matrix of significance values for pairwise comparisons across manufacturers .94

Table 6.1. Likelihood ratio test for the logistic regression terms 100

Table 6.2 Confusion Matrix for training dataset 104

Table 6.3 Confusion Matrix for test dataset 105

Table 6.4 Likelihood ratio test for final linear model terms 107 


\section{List of Figures}

Figure 1.1. New Zealand pop-up information display example

Figure 3.1. Histograms of session length where at least one pop-up occurred by each of the five manufacturers 51

Figure 3.2. Quit on pop-up rates with manufacturers' pop-up onset times overlaid .. 53

Figure 3.3. Histograms of session length where players quit on a pop-up by each of the five manufacturers 54

Figure 3.4. Histogram of all session lengths for AGT and Aristocrat machines 56

Figure 3.5. Histograms displaying session lengths for IGT machines 59

Figure 3.6. Histograms displaying session lengths for Konami machines 60

Figure 3.7. Histograms displaying session lengths for Star Games machines 61

Figure 4.1. Graph displaying main effect of venue type 70

Figure 4.2. Graphs displaying interaction between net winnings and manufacturers . 72

Figure 4.3. Bar graphs displaying interaction between the three regions and time of day

Figure 4.4. Bar graphs displaying interaction between the net winnings and time of the day 76

Figure 4.5. Bar graph displaying interaction between number of pop-ups and manufacturers

Figure 4.6. Bar graph displaying interaction between number of pop-ups in a session and net winnings 78 
Figure 4.7. Bar graph displaying interaction between number of pop-ups seen and time of day .79

Figure 4.8. Bar graph displaying interaction between rate of play and net winnings ...80

Figure 4.9. Bar graph displaying interaction between rate of play and manufacturers 81

Figure 5.1. Graph showing elective display access rate across the manufacturers with popup onset times overlaid 95

Figure 6.1. Histogram displaying the distribution of number of elective displays accessed .99

Figure 6.2. Histogram displaying the distribution of rate of elective displays accessed .99

Figure 6.3. Line graph showing the relationship between the total hours played and log odds of at least one elective display access 101

Figure 6.4. Bar graph showing main effect of venue type on the log odds of accessing elective displays across the five venue types 102

Figure 6.5. Bar graph showing main effect of manufacturer on the log odds of accessing elective displays across the five manufacturers 103

Figure 6.6. Two bar graphs showing the interaction between venue type and manufacturer 108

Figure 6.7. Bar graphs showing the interaction between venue type and region 110

Figure 6.8. Scatterplot showing the predicted and observed elective per hour rates for the train dataset 112 
Figure 6.9. Scatterplot showing the predicted and observed elective per hour rates for the test dataset 113 


\section{Acknowledgements}

I would firstly like to thank my supervisor Dr. Anne Macaskill for her guidance, knowledge and expertise during this project and Dr. Maree Hunt for all her input and valuable advice throughout. Funding for my studies was gratefully received from Victoria University of Wellington through the Masters by Thesis Scholarship. A special thanks must go to the Department of Internal Affairs for their cooperation in providing the dataset used in the analysis. The statistics behind this thesis would not have been possible without the patient help of Lisa Woods, our fabulous statistics advisor. Sorry for the endless dead ends, we made it in the end though!

To my friends, office mates and everyone who has helped me along the way, thank you for the lunch time catch ups, the decaf coffees, the talks to keep me motivated and the walks to get treats. Your support has been invaluable.

Last but not least, a massive thanks to my family, to whom this thesis is dedicated. Mum, Dad, Alanna and our dog Holly, this thesis would not have been possible without all of you. Thank you for the pick-ups when I was having a meltdown, the delicious food to keep me going and the late-night proof reads, I'm sure you had better things to do, but I hope you now know more than would ever want to on logistic regression! Your endless love, support, and ability to always be there when I needed you was the only reason this thesis made it to submission. 


\begin{abstract}
Electronic gaming machines (slot machines) contribute to problem gambling in New Zealand and worldwide. Information displays are one harm minimisation feature of New Zealand electronic gaming machines that has been investigated in recent years. New Zealand gamblers see two types of displays: interruptive pop-up displays after a set period of time has passed, and elective displays if a player presses an information button. These displays inform the gambler about their current session with the intention that they will facilitate informed decisions about continuing play or quitting. The current study used a week-long set of electronic gaming machine data from across New Zealand to run an exploratory investigation into the utility of these information displays. We first observed that fewer than $2 \%$ of pop-up displays resulted in people quitting. On this basis it is unlikely that interruptive pop-up displays are meaningfully reducing harm. Our analysis also revealed timing differences in how pop-up displays are scheduled on machines produced by different manufacturers. The likelihood of quitting on a pop-up was influenced by complex interactions of machine and session characteristics, however these effects were small. Secondly, our investigation of elective displays also identified a low rate of access, indicating they are also unlikely to be working effectively as a harm minimisation tool. Analysis revealed players' likelihood of accessing an elective display was mostly influenced by the venue type they were in and the manufacturer of the machine. Possible recommendations to improve both types of displays include changes to message content, scheduled timing and visual features.
\end{abstract}




\section{Chapter 1: General Introduction}

Despite gambling being a legal form of entertainment, across the world it is known to be problematic for a significant group of people. The social and financial consequences for those individuals can be devastating (Landon, Thorne, Palmer, Page, $\&$ Abbott, 2010). The worldwide prevalence of problem gambling is between $0.12 \%$ and 5.8\% of the population (Calado \& Griffiths, 2016). In New Zealand these numbers are similar, with $0.2 \%$ of the population fitting the criteria for problem gambling while an additional $5.8 \%$ are in a moderate gambling risk group (Abbott, Bellringer, \& Garrett, 2018).

Problem gambling is associated with a large number of significant negative impacts. These include psychological distress, depression, anxiety, exacerbated alcohol and tobacco use, and physical health problems. These are found alongside other negative impacts such as deprived housing situations, neglect of children, poorer relationships and worse overall quality of life (Landon et al., 2010; Lin, Casswell, \& You, 2008). Lin et al. reported that mental well-being was reduced for $2.4 \%$ of New Zealanders as a result of gambling. While some of these impacts affect only the problem gamblers themselves, there are also many negative impacts for others. Close family and friends nearly always feel the negative economic and social consequences of a person's problem gambling, and these impacts can reach far into the extended community, due to an increase in gambling-related crime (Ministry of Health, 2010). Lin et al. reported that in New Zealand approximately 10,000 people engaged in illegal activity within a year because of gambling.

While these statistics about problem gambling include the harms caused by many modes of gambling, play on electronic gaming machines (EGMs; also known as pokies, slot machines or one-arm bandits), is of specific interest to the current research. 
Research tends to agree that worldwide EGM gambling is the mode of gambling most often associated with harm (Dowling, Smith, \& Thomas, 2005; Echeburúa, Báez, \& Fernández-Montalvo, 1996; Productivity Commission, 2001; Smith \& Wynne, 2004; Turner \& Horbay, 2004; Wynne, 2002). In a study of problem gamblers, Binde, Romild, and Volberg (2017) found that participants' most popular form of regular gambling was on EGM machines, highlighting the association between problem gambling and EGMs. This relationship is seen in New Zealand with over two in every five people (43\%) who regularly play EGMs, fitting the problem gambling classification, or belonging to a moderate risk problem gambling group (Dowling et al., 2005; Gray, 2011). This is compared to $15 \%, 6 \%$ and $9 \%$ of regular players fitting the moderate or problem gambling classification for racing, lottery ticket and instant kiwi scratch ticket type gambling, respectively (Gray, 2011). Information from problem gambling interventions collated by the New Zealand Ministry of Health show that over half of all clients presenting for treatment, identify EGM play as their main form of gambling (Ministry of Health, 2018).

\section{Electronic Gaming Machines}

The forerunners to the modern EGMs were first invented in 1895 (Dowling et al., 2005). These initially had a basic mechanical design and the machines themselves were often referred to as 'one-arm bandits'. EGMs have evolved significantly over the years to become the sophisticated computer-operated systems that we see in venues today (Dowling et al., 2005). Modern EGMs utilise advanced gaming software technology and are designed to incorporate various visual and auditory characteristics and features known to reinforce and facilitate further play (Griffiths, 1993). 
EGMs typically display five reels of symbols that, when activated, spin on a screen until they slow to a stop revealing various symbol outcomes for that turn. The outcome of each spin is based on predetermined probabilities that are unaffected by the player. The combination of symbols the spin lands on communicates whether the player has won money for that turn. There are different winning values associated with different combinations of symbols and differing bet amounts yield different returns, making it a complex game. This is further complicated by the option to wager on multiple lines, allowing the player to not only wager that there will be consecutive matching symbols on one line (e.g. the row of five symbols in the middle of the display), but they can also pay more to wager the possibility of consecutive symbols appearing across multiple different lines (e.g the top row of symbols or a diagonal pattern of symbols). When a gambler wagers on multiple lines they pay more to make multiple bets on one spin.

Given EGMs' contribution to problem gambling, there has been an unsurprising worldwide increase in research investigating harm reduction tools. Harm reduction strategies investigated have ranged considerably. One intervention researchers examined was placing warning signs in gaming rooms near EGMs. These warning signs had limited effect compared to dynamic warnings appearing on-screen during gaming play (Monaghan \& Blaszczynski, 2007). This conclusion was also drawn by Harris and Griffiths (2017) and Ginley, Whelan, Pfund, Peter, and Meyers (2017) in their review article assessing the available literature on the same tool. A review by Tanner, Drawson, Mushquash, Mushquash, and Mazmanian (2017) looked at mandatory cashing out after a specified amount of time had passed, indicating this had limited impact on time and money spent playing. They also concluded one of the more promising harm minimisation strategies was patron self-exclusion. In addition, Ladouceur and Sévigny 
(2009) found extra visible on-screen clocks had minimal impact but that more visible cash total displays, led to greater control of gambling behaviour. Sharpe, Walker, Coughlan, Enersen, and Blaszczynski (2005) found that imposing maximum bet sizes was more effective than slowing spin speed and removing large note acceptors. One final harm minimisation tool studied in the literature was information displays and this is the focus of the current study.

\section{Information Displays}

The current study focused on information displays presented on EGM screens. These come in two forms: interruptive displays, which appear during gambling sessions forcing a brief break in play while presenting the gambler with information, and elective displays, which contain similar information but appear only when a player chooses to access them by pressing a button. New Zealand EGMs are required to have both forms of information displays. Information displays are a popularly studied tool with researchers internationally exploring their potential to increase responsible gambling (Ladouceur \& Sevigny, 2003; Schellink \& Schrans, 2002; Wynne \& Stinchfield, 2004).

There are a number of different ways that information displays - mainly pop-up displays - have been studied. Research approaches range from experimental research conducted in laboratories (Jardin \& Wulfert, 2009; Ladouceur \& Sevigny, 2003; Monaghan \& Blaszczynski, 2010) to focus groups (Landon, Palmer du Preez, Bellringer, Page, \& Abbott, 2016; Palmer du Preez et al., 2014; Schellink \& Schrans, 2002). As well as research conducted in simulated (Cloutier, Ladouceur, \& Sévigny, 2006), and real (Gainsbury, Aro, Ball, Tobar, \& Russell, 2015; Palmer du Preez et al., 2014; Schellink \& Schrans, 2002) gaming lounges. 
There are variations across pop-up display literature in regard to: the message content; the frequency with which displays are presented to the gamblers; and the way their effectiveness is measured. The general findings of this research are that pop-ups can be effective at helping people to gamble more responsibly, however, the extent to which they are effective depends on how frequently gamblers see messages, and the type of content they display. Review articles by Gainsbury, Abarbanel, Philander, and Butler (2018); Ginley et al. (2017) and Harris and Griffiths (2017) specifically assessed the harm minimisation tool of interruptive pop-up displays. Gainsbury et al. suggested that the extent to which these warning messages were effective depended on the group which received them, adding another level of complexity to finding an optimal content type. Harris and Griffiths explored the many different message content types that have previously been studied. They identified there was evidence of pop-ups reducing harm but conclusions were difficult as studies were conducted in laboratories rather than naturalistic gaming lounges, and the majority of studies were conducted on nonproblem gamblers. Ginley et al. found general support for the use of warning messages as a harm reduction tool and also explored how the mode of message display (placement, framing etc.) influenced the impact pop-ups had.

Information displays in New Zealand. In New Zealand, the risks associated with gambling are recognised by the government. The Ministry of Health is responsible for preventing harm caused by gambling (Ministry of Health, 2010) and the Department of Internal Affairs is responsible for regulating the gambling sector, ensuring people and entities are complying with legislation. Since 2003, all gambling activities have been regulated by the Gambling Act (2003). A purpose of this Act is to keep New Zealanders safe from the harms that gambling can cause. This involves ensuring that gambling 
growth is controlled, that all gambling is carried out in a responsible way and profits from EGMs go back into the community (Department of Internal Affairs, 2013).

The Gambling Act (2003) was given Royal assent on the $18^{\text {th }}$ of September 2003, but only a few specific provisions came into force on that date. A consultation document relating to harm minimisation regulations was released following this. Of relevance to the current research, these proposed regulations required inclusion of both pop-up, and elective player information displays.

Following consultation, it was decided that both pop-up and elective information displays would be required on all new machines from 1 July 2005 and on all machines by 1 July 2009. The technical requirements for these information displays were laid out in the Appendix to the National Standards (Department of Internal Affairs, 2016).

A Cabinet Paper described the rationale behind the inclusion of information displays (Cabinet Policy Committee, 2003). The main motivation was to reduce the likelihood that people could gamble without being fully informed about how the games worked and how much they were losing. Based on the Department of Internal Affairs' (2005) research into people's beliefs about gambling activities, the government identified New Zealanders were unaware of how much they were losing. In 2000, $17 \%$ of EGM players believed they had won money overall on EGMs in the last 12 months, this increased to $22 \%$ in 2005 . They noted that, given EGMs are programmed so, on average, gamblers lose more money than they win (i.e. the machine makes a profit), it was evident that many people had false ideas about how often they were winning. This same piece of research asked participants about their use of systems or skills to improve their chances of winning. In 2000, $8 \%$ of EGM players indicated they used a special skill or system, in 2005 this number was 5\%. Given that each spin on an EGM is 
independent of all other spins and only chance plays a role in the outcome, beliefs about having skills that could improve the chance of winning are false.

Additional evidence supporting the inclusion of pop-up information displays presented in the Cabinet Paper came from the study conducted by the Department of Internal Affairs from 1985-2000 (Department of Internal Affairs, 2001). This study asked people how warnings would affect the frequency of their gambling. Information that 'the house always wins' led to $18 \%$ of participants saying they would gamble less frequently. Warning information about length of time/money spent playing, led to $26 \%$ of participants indicating they would gamble less frequently. The current regulations require the use of warning information. The Cabinet Paper also reported feedback from other jurisdictions where pop-ups had been implemented, including Nova Scotia, which suggested they were proving effective. There was a lack of evidence however, supporting the regulation details. That is, the Paper provides no rationale for the specifics such as the required timing of displays and specific choice of message content. The Cabinet Paper also provided no discussion of literature evaluating the inclusion of pop-ups or elective displays, as most of the research in this area came out after 2003.

The regulations specify that "Player information, must be displayed during a player's session of play at random intervals. The random intervals must be triggered during a set period of time not less than five minutes. The set period can occur between 0 and 30 minutes, but the actual display of the information must not exceed 30 minutes after the start of the session of play or the cessation of the last interruptive display of player information." (Department of Internal Affairs, 2016, p. 17). This means that interruptive pop-up displays must appear at random intervals during sessions of continuous play, such that uninterrupted play cannot go for longer than 30 minutes. The manufacturers have discretion when implementing these regulations so long as these 
requirements are met. There are five manufacturers of EGMs in New Zealand: AGT, Aristocrat, IGT, Konami and Star Games.

Figure 1.1 provides an example of the interruptive pop-up display. They are designed to force a brief break in gambling play by pausing the game for a minimum of 15 seconds and are required to display the following information:

a) the duration of the session shown in hours and minutes;

b) the amount in dollars and cents that the player has spent during the session;

c) the player's net wins or net losses during the session; and

d) a question asking the player whether they wish to continue playing or quit (Department of Internal Affairs, 2016, p. 16).

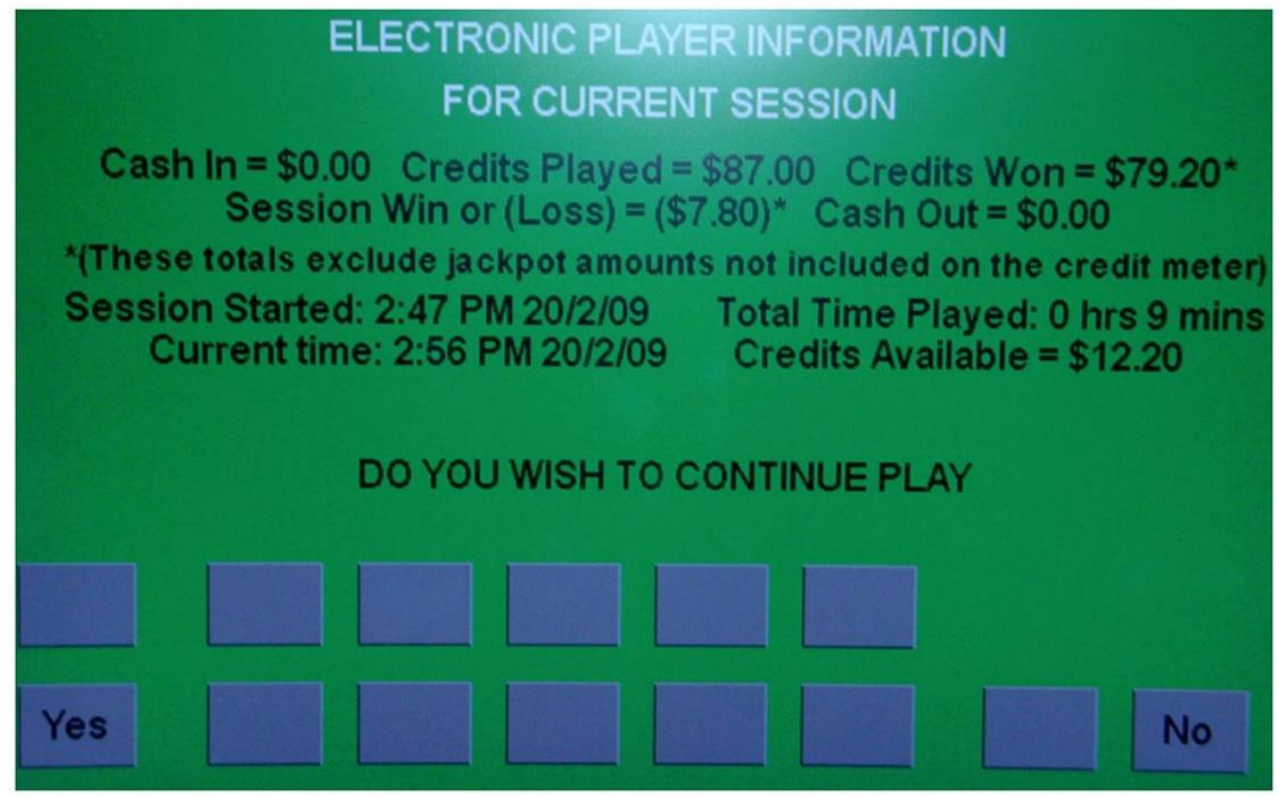

Figure 1.1. New Zealand pop-up information display example (Landon et al., 2016, p. 50)

The other form of information display required by the Department of Internal Affairs (2016) is the elective display. These displays contain the same information as 
the pop-up displays, along with additional game information. This additional information includes the odds of winning the game, the five top and bottom winning combinations (winning value), the maximum and minimum spend per bet, and the average winnings paid out to players over a certain number of plays. This information may be aimed at reducing false gambling beliefs, as the government provided this as a reason for requiring information displays. This information can be accessed at any time during play through pressing a button and the session information must be continuously updated. EGMs are required to record how many times these elective information displays are accessed (see below). To ensure the recorded total is a true reflection of people attempting to view the information display, it is required that this screen does not provide access to any other pages (Department of Internal Affairs, 2016).

\section{Interruptive Pop-up Displays}

New Zealand pop-up information displays have a unique combination of message content and timing making them distinct from pop-ups examined in most previous studies. For this reason, the current study investigated how effective New Zealand pop-ups are and whether there were ways to make them more effective. Despite distinctions between New Zealand pop-ups and pop-ups found in other countries, international research can still provide useful insights.

The first important point highlighted in the literature is that pop-ups generally consist of a number of key elements. The first is that pop-up displays are an opportunity to provide gamblers with information while they are playing the EGMs (the content of this information differs significantly across the literature). The second element is providing a compulsory break from play which only ends once the pop-up has disappeared. The length of this break differs across the literature, however this aspect is 
common to all pop-ups. The third element that New Zealand pop-ups have but some others do not, is a question which gives the gambler an opportunity to quit their session.

Research on pop-ups providing information. There are key differences across the literature regarding what information is provided to players through the pop-ups. The three key different types are: information to reduce false cognitions, self-appraisal messages and session feedback.

Reducing false cognitions. The most common pop-up message content in the literature aims to reduce false cognitions about the control gamblers have over spin outcomes and the odds of winning games. While many factors contribute to problem gambling, false and irrational cognitions are widely accepted as one such factor.

Delfabbro and Winefeld (2000) found in a sample of gamblers, 14\% of statements made while playing gaming machines were irrational. When specifically observing gamerelated statements the percentage increased to $75 \%$, suggesting gamblers' irrational thinking was specific to game play.

Blaszczynski and Nower (2002) explored whether distorted schema was a cause or an effect of gambling. They suggested that strong, distorted cognitive schemas about the chance of winning were developed over repeated gambling sessions when gamblers made incorrect attributions about the control they had over the outcome of each spin. Blaszczynski and Nower suggested this leads to beliefs in using skill to influence outcomes and players feeling their chance of winning was higher than reality. This indicated that, while false cognitions seemed to be associated with problematic gambling they could just as much be an outcome of it rather than the cause.

This association between gambling and false beliefs is further evidenced by treatment targeting erroneous beliefs and correcting distorted cognitions. Research in 
this area indicates this treatment reduces gambling problems (Sylvain, Ladouceur, \& Boisvert, 1997). It must be noted, however, that this treatment also included problemsolving skills training, social skills training, and relapse prevention, so it was not clear how much of an effect reducing false cognitions had on its own.

The literature on erroneous EGM beliefs in the development and maintenance of problem gambling provides suggestive evidence that pop-ups aiming to reduce false beliefs could reduce gambling harm. Pop-ups aimed at reducing false cognitions tend to include information about odds of winning a particular game and remind players that spin outcomes are independent and not influenced by previous spins. Although several studies have found evidence of this type of pop-up having positive effects on gambling, other studies find the contrary.

In an example of a study suggesting pop-ups aimed at correcting false beliefs are effective, Jardin and Wulfert (2009) had participants play a chance-based computer game. The experimental condition group were exposed to pop-ups every three trials which included a message such as "You cannot control the outcome of this game". The group exposed to these messages played significantly fewer trials and spent significantly less money, compared to those in groups who saw neutral messages such as "The Wheel of Fortune was invented in 1975", and those who saw no messages. Jardin and Wulfert (2012) replicated their finding in a similar study a few years later. Benhsain, Taillefer, and Ladouceur (2004) also found that pop-up messages describing the independence of gambling events reduced both erroneous verbalizations and length of play compared to neutral messages on a computerized roulette task.

This is the extent of studies finding a positive effect of false cognition correcting pop-up messages. The commonality between these successful pop-ups was that they 
appeared very frequently. Jardin and Wulfert (2009) had their pop-up messages appear every three trials while both Benhsain et al. (2004) and Jardin and Wulfert (2012) had them appear every five trials. This is a possible explanation for these specific studies finding pop-up messages reduce gambling behaviour.

Other research investigating pop-ups targeting erroneous beliefs found that they altered gambling cognitions but did not alter gambling behaviour. Reviews by both Gainsbury et al. (2018) and Harris and Griffiths (2017) came to the same conclusion that while they correct irrational beliefs, empirical evidence of gambling behaviour changing, is minimal. Cloutier et al. (2006) had participants play on video lottery terminal screens in a simulated bar. The researchers measured beliefs about gambling before and after participants played the game. Researchers observed a greater decrease in false beliefs in the group that received pop-up messages targeting false beliefs ("No matter how you play, you cannot influence the outcome of the game") compared to the group that received the word "Pause". Despite this, researchers found no effect of message type on the actual number of games played.

Similarly, Ladouceur and Sevigny (2003) had participants play video lottery terminals where they either received pop-ups about the chances of winning, a break or no interruptions. They found that messages correcting false beliefs had no effect on gambling behaviour. A study by Steenbergh, Whelan, Meyers, May, and Floyd (2004) provided further evidence that messages targeting false cognitions can change beliefs but not gambling behaviour. A pop-up targeting false beliefs appearing at the start of a gambling session led to an increased understanding of gambling risk and a reduction in false beliefs. Despite this, the way people gambled on electronic roulette remained unchanged in response to these messages. 
It is useful to note that both Cloutier et al. (2006) and Ladouceur and Sevigny (2003) had pop-ups appearing every 15 trials and Steenbergh et al. (2004) had only one pop-up at the start of gambling sessions. The pop-ups in these studies were more infrequent compared to pop-ups in studies that found these types of messages reduced gambling behaviour. It is possible that low rates of exposure to these messages is enough to influence people's cognitions and beliefs about gambling. However, it may be that for this type of message to be effective at influencing gambling behaviour, exposure to these needs to occur more frequently than every 15 spins.

Health related warning labels aimed to improve health outcomes are relevant when assessing the effectiveness of using pop-ups to reduce false cognitions in gambling. Looking specifically to other health-related addictions, alcohol and tobacco are both addictive substances where health warning labels have been implemented to reduce risky behaviour. This research provided evidence both for and against the ability of warning labels to influence and change consumer behaviour (Hammond, 2011).

In a review of alcohol and tobacco warning labels, Stockley (2001) suggested there were two aims of health-related warning labels. The first was to increase consumer awareness about issues arising from excessive or inappropriate use. The second aim was to modify consumer behaviour with this education, therefore preventing harmful consequences. Stockley presented evidence that warning signs increased awareness and knowledge of the health effects of alcohol consumption, but flow on effects modifying the risky behaviour were non-existent. This links closely with the findings within the gambling domain reviewed above. A reduction in false beliefs also did not lead to a change in behaviour. Engs (1989) used the idea of the Health Belief Model to explain why informing someone a behaviour is harmful or risky is not sufficient to change a person's behaviour away from what is harmful. Engs explained that initiating changes 
in human behaviour is a very complex process and will only occur under a certain set of circumstances, one of which is people feeling personally susceptible to the possible negative impacts. These circumstances can be hard to achieve.

There is divergence between knowing risks and refraining from engaging in risky behaviour across a wide range of health domains. These include; alcohol abuse (Goodstadt \& Caleekal-John, 1984), drunk driving (Greenfield, 1997 as cited in Stockley, 2001), and smoking (Robinson \& Killen, 1997; Engs, 1989). A meta-analysis on warning labels in general, including studies of warning labels on addictive substances like alcohol and tobacco as well as other warnings such as those relating to chemicals, pools, saws and electrical generators, can provide further information (Argo \& Main, 2004). They found warnings had much larger effects on measures of knowledge, than they had on measures assessing behavioural compliance (outcome behaviour). This further supported the argument that knowledge of risks, is not synonymous with influencing behaviour.

More recent reviews such as Hammond (2011) have slightly different findings. Hammond examined the effects of updated health warning labels. This research indicated warning signs could reduce smoking. Hammond's review highlighted that the size and design of the warning were important, noting that large, prominent health warnings on the face of packages distinct from the rest of the text were most effective. He also commented that stronger emotional reactions could be induced by using high contrasting colours and pictures.

A disadvantage of pop-ups aiming to reduce irrational cognitions is that messages are subject to over exposure effects. Evidence in literature about health warning messages suggest health warnings are much more effective soon after their 
implementation and slowly decline in effectiveness over time (Hammond, 2011). Rotating messages can reduce this wear out effect, but ultimately the limitation cannot be removed entirely. This was supported by a large-scale survey conducted in Canada. They found the majority of smokers indicated informative warning labels introduced years earlier had lost their effectiveness over time (Environics Research Group as cited in Monaghan \& Blaszczynski, 2009).

This literature provided contradictory findings as to the effectiveness of warning messages that aim to inform a recipient of risks, whether it be gambling or other health domains. A possible explanation for these differences appears to be the frequency in which the recipient is exposed to the informative message. In the research, the pop-up messages found to be most effective were those that appeared every five spins or less, and the most effective health warnings were those that were likely to be seen upon every use of the product. Perhaps infrequent exposure is enough to change false beliefs and accurately inform people of risks, but in order to change behaviour high frequency exposure is required with this type of information. This is further complicated by wear out effects of exposure occurring more quickly for messages that are seen frequently.

New Zealand pop-ups differ from those typically designed to reduce false cognitions despite this being identified in the Cabinet Paper as a key reason for making pop-ups a requirement. While the feedback information provided on pop-ups in New Zealand could reduce overestimations of win amounts, this literature highlighted more targeted ways of doing this. It remains unclear if New Zealand pop-ups would benefit from this type of messaging as their presentation is on a very infrequent schedule (at least one per 30 minutes). 
Self-appraisal. Another main pop-up message content type is self-appraisal. Self-appraisal messages are designed to help players reflect on and evaluate their own actions and, following this reflection, regulate their behaviour (Monaghan, 2009; Monaghan \& Blaszczynski, 2009). Self-monitoring is one of the more effective ways of achieving behavioural change. In the context of gambling, reminders to evaluate one's behaviour and compare it to intentions (how much time and money they were planning on spending) can facilitate responsible gambling behaviour (Monaghan, 2009; Monaghan \& Blaszczynski, 2009). Monaghan and Blaszczynski argued that an additional advantage of this type of messaging is its resistance to over-exposure and wear-out effects. Self-appraisal questions ask players to reflect on their current situation. As the situation will be different each time a pop-up appears, this type of message does not have the same repetitive nature as informative messages.

New Zealand pop-ups have a self-appraisal element to them: the question "Do you wish to continue?". This requires the player to decide whether they want to continue or not. It is slightly different to the self-appraisal messages seen in the literature which tend to ask the player to consider what they should or need to do by questions such as "Do you need a break?". These should or need questions are more encouraging of responsible gambling, as what people want to do is often not the choice that will be the best for them in the long run, more so for people with addictions.

Monaghan and Blaszczynski (2010) conducted an in-vivo study on self-appraisal messages using computer EGM simulations. They compared the effectiveness of informative, self-appraisal and control/blank pop-up message types. The self-appraisal messages used included "Do you know how long you have been playing? Do you need to think about a break?" and "Have you spent more than you intended?". They found that self-appraisal pop-ups, compared to informative and control pop-ups, had the 
greatest impact on within-session thoughts. When asked about their expected influence when playing on a real EGM, they found self-appraisal messages had the greatest influence on players' predicted awareness of play time, play duration, and likelihood of taking a break.

Another similar study conducted by Gainsbury et al. (2015) also compared informative messages with self-appraisal pop-up messages: "Have you spent more than you can afford?" and "Is money all you are losing?". They examined the effect these messages had on recall of content, the impact they had on thoughts, expected EGM play and lastly, people's perceptions of usefulness. They found self-appraisal message content was recalled significantly better than informative messages. Despite this, there was no difference in self-reported behaviours and self-reported thoughts following each type of pop-up, suggesting self-appraisal messages had no advantage in overall impact.

The conflicting results of both of these studies need to be treated with caution as they are both measured by self-report. Without directly measuring gambling behaviour it is difficult to conclude how effective these self-appraisal messages were. Factors such as the exact wording of the question could influence these self-reported answers and therefore account for these different findings. Research in the gambling field has demonstrated that self-report measures can differ from objective measurements, and a combination of both actual measurements, and self-report should be used to gain an accurate understanding of gambling behaviour (Auer \& Griffiths, 2017).

As there is a lack of research into the impact that self-appraisal messages have on gambling behaviour, looking to other health related fields is useful. There is evidence that reflecting on one's self can lead to greater behavioural change in smokers. Chua et al. (2011) looked at participant smoking cessation, comparing tailored, 
personalised messages about the participant's history, with generic informative messages about smoking. Using MRI machines, they found that personal messages increased activation in self-related processing neurological regions of the brain and, that activation in these self-related areas predicted quitting four-months later. This showed messages encouraging self-reflection can reduce engagement in addictive behaviours pointing to their potential utility in the gambling domain.

Overall, looking at self-appraisal pop-up messages there is not sufficient evidence to conclude whether or not they are effective. It appears that this type of message has promise in other domains so may potentially contribute to any effect New Zealand pop-ups may have.

Feedback. The final message content type seen in the pop-up literature is feedback about the player's current gambling session. New Zealand pop-up messages contain time and spending information, and also provide gamblers with a question about whether they wish to quit. This type of content is thought to be effective because it provides accurate information about the immediate session, enabling players to make well-informed decisions about whether they should keep gambling (see discussion above). Across the literature these types of pop-ups tend to include a question gamblers can respond to following the feedback, about whether they wish to continue playing or not. This answerable question does not appear to be found in other message content types. The feedback literature branches into two key categories. The first was feedback in the form of a reminder that a previously set monetary or time limit had been reached. The second was current session time or money spent information appearing after a certain amount of time had passed. 
Research on messages reminding players of pre-set limits, tended to find increases in people's adherence to these limits. Questions asking whether they wanted to quit or continue playing once this limit was reached were included. Stewart and Wohl (2013) had participants play on simulation EGMs in a virtual reality casino setting. They found players who received pop-ups reminding them they had reached the monetary limit they had set, had greater adherence to monetary limits compared to those who did not receive these pop-ups. This effect was replicated by Wohl, Gainsbury, Stewart, and Sztainert (2013).

Auer and Griffiths (2013) also conducted a similar study looking at online, high intensity gamblers. They found pre-set limit reminders reduced both monetary spending and play duration. Auer, Hopfgartner, and Griffiths (2018) replicated the same finding using reminders that $80 \%$ of their pre-set monetary limit had been reached.

Kim, Wohl, Stewart, Sztainert, and Gainsbury’s (2014) found additional support for limit setting influencing play time. Unlike other studies, Kim et al. only used one pop-up to set a time limit and once the pre-set limit was reached, no second pop-up informed them that their time limit had been reached. Despite the lack of a second reminder, they still found that players who set a limit, played for less time than those who did not. This indicated that perhaps the act of setting a limit was the driving force behind pre-set limit reminders reducing play rather than the reminder itself.

Hollingshead, Wohl, and Santesso (2019) tested the possibility of pre-set limit reminders being improved by also containing personalised feedback information. They found this made no improvement to how effective pre-set monetary limit pop-ups were, indicating the addition of more information is not necessarily a useful way to increase their effectiveness. It should be noted limit adherence was measured as a binary 
outcome, meaning people spending one cent over their limit were classified as not adhering to their limit just as much as someone who spent twice as much as they intended to. Information about the extent to which participants over-spent and therefore the potential problem that they pose to themselves was not captured in this study. A manipulation check within their study also indicated the lack of improvement was because people did not read the content of the pop-up messages. This indicated a potential limitation of all pop-up messages.

The other form of feedback style pop-ups found in the literature were those that provided up to date session information at predetermined times, unrelated to reaching limits gamblers had set for themselves. These studies suggested that gamblers tend to notice these pop-ups, but they have limited impact on responsible gambling behaviour. The first study investigating session information pop-ups was conducted by Auer, Malischnig, and Griffiths (2014). They used online gambling website data to compare session quitting before and after the addition of a pop-up feature. The pop-ups appeared after a participant had played 1,000 games with the words "You have now played 1,000 slot games. Do you want to continue? (YES/NO)". This study found an increase in the likelihood of players quitting when exposed to the pop-up compared to when the popups were absent, however this increase was very small.

Wynne and Stinchfield (2004) also looked at how session information pop-ups impacted play, this time without an additional question of whether or not they wished to continue play. They compared responses from people who had played on new machines with this responsible gaming measure in place, with those who had only played the old machines without these. While pop-ups were noticed by most people, only a few players reported using them to keep track of their time and spending, and the majority suggested they were not effective. 
Schellink and Schrans (2002) came to a similar conclusion in their study which included a quit or stay playing option following the pop-up. They also found low risk players made up a high proportion of the few players who indicated these pop-ups were effective compared to high risk players. The results of both of these studies must be treated with caution as participants' gambling behaviours were not directly tested, only self-report measures were taken.

The limited effectiveness of pop-ups displaying session information can also be seen in Auer and Griffiths' (2015) study. They compared the effect of pop-ups displaying session duration information with an 'enhanced' pop-up which included: session feedback, normative feedback, information to correct false beliefs and selfappraisal messages. They found that the enhanced pop-up was more effective than the session information pop-up. It was unclear as to what elements, or combination of elements of the enhanced pop-up were responsible for the improvement but showed session information alone was not the most effective option.

The final study of particular relevance to New Zealand pop-up content, is a study conducted in New Zealand commissioned by the Ministry of Health looking at New Zealand pop-ups, using mixed methods such as focus groups and surveys to assess their effectiveness (Palmer du Preez et al., 2014). They found half of gamblers attended to information on pop-ups and about a quarter reported these displays assisted them in controlling their time and money spent gambling (slightly - moderately). A supplementary in-venue observation study showed that $22 \%$ of people cashed out following a pop-up appearing in pubs (this included players leaving and changing machines; Palmer du Preez, Landon, Bellringer, Garrett, \& Abbott, 2016). There was anecdotal evidence that after having seen a pop-up, people played on to finish their 
credits and left (this was not measured, as it was not an immediate response but could have been influenced by the presence of a pop-up).

In a review of optimal content for pop-ups, Monaghan and Blaszczynski (2009) comment on the potential issues with temporal and monetary reminder pop-ups. They suggest that without a central tracking system where people can be tracked as they change machines there is no benefit to reminders of this kind. They indicate they may even be of detriment with the potential to inform players they have spent less time and money than they actually have, encouraging further play.

This research provides evidence that pop-ups containing feedback can be an effective tool for controlling gambling. Feedback in the form of reminders that pre-set limits had been reached were more effective than those that provided information about the current session. New Zealand pop-ups include the latter but could potentially benefit from the addition of players setting limits and being reminded of these.

Research on pop-ups as a break. While the pop-up messages can be used to communicate information, the content of which tends to differ throughout the research, they also have a second important function that is common to all the pop-ups. This is, they provide players with a compulsory break from their gambling session. Pop-up displays appear across the whole screen pausing the game, forcing the player's attention away from the game being played.

A known contributor to problem gambling is the state of dissociation that people get into when they engage in addictive behaviours (Jacobs, 1988). This state of dissociation is generally described as a loss of control caused by players becoming totally absorbed in the game that they are playing. It is thought players lose track of their gambling session, play for longer than intended and spend more money than 
planned (Monaghan, 2008, 2009). Such states can also be described as a narrowing of attention towards the game, feeling like the player is 'outside themselves' and in a trance (Jacobs, 1998).

The break element of pop-ups is thought to increase responsible gambling because pop-ups can break this state of dissociation (Johnston \& Dark, 1986). The idea is, the small break from play allows players to refocus their attention to their environment and what they are doing, and make an assessment of whether they are spending more time and money than intended (Monaghan, 2009). The state of dissociation is linked closely with problem gambling, with increasing levels of dissociation being linked to increasing levels of problem gambling severity (Jacobs, 1988). Given this, it appears that this break would have its greatest influence on those experiencing problems with their gambling compared to recreational gamblers, which is one aim of harm minimisation tools (Monaghan, 2009).

The literature provides evidence for the break element of these pop-ups being the driving factor in pop-ups helping participants play fewer games. Ladouceur and Sevigny (2003) investigated this, using a pop-up with a message experimental condition, a break only experimental condition, and a no interruption control condition. They found that both the break only, and message condition reduced gambling compared to no interruptions, with no significant difference between the break and the message conditions. Cloutier et al. (2006) replicated this pattern of results. These studies indicated that the break was driving the reduction in games played with messages providing no advantage.

This is, however, opposed by the finding of Jardin and Wulfert $(2009,2012)$ who compared informative messages, neutral messages and no interruptions. They 
found that informative messages led to a reduction in bet size and time playing compared to both neutral messages and no interruptions. They also found no difference between the neutral message condition and the no interruptions condition. This indicated, unlike the above two studies, that the break element alone was not enough to disrupt gambling.

A possible explanation for these differences could again be the frequency of the pop-ups. As noted above Jardin and Wulfert (2009, 2012) had pop-ups appear between every three and five spins in their studies. It is likely that these studies, which found no effect in the break condition, did not allow enough time between each pop-up for a player to head towards a state of dissociation. This meant that when the break in play occurred, no effect was seen.

The studies by Cloutier et al. (2006) and Ladouceur and Sevigny (2003) which found an effect in the break condition, had pop-ups appear every 15 spins. In these studies, it is possible that there was more chance of a player heading towards a state of dissociation. When a break appeared, it was able to have some effect on dissociation and therefore reduce play. It is possible that when a state of dissociation was not attainable, having a message appear is necessary to reduce gambling. However, it is possible when more time between pop-ups occurs and a state of dissociation is more likely to be entered into, the break element is able to exert its effect, resulting in the message element providing no additional advantage.

Stewart and Wohl (2013) further evidenced the role of dissociation in how popups function. Their analysis revealed that pop-ups reduced people's levels of dissociation and level of dissociation mediated the relationship between someone's problem gambling severity and their failure to adhere to limits while gambling. This 
suggested that breaking play and therefore reducing dissociation was a way to reduce gamblers from overspending.

In addition to these studies, Blaszczynski, Cowley, Anthony, and Hinsley (2016) investigated breaks in the absence of a warning message. They found that following breaks, craving increased, and the longer the break, the more craving the participants experienced. It should be noted the breaks in this study ranged from three minutes to eight minutes which is much longer than the seven second breaks in both Cloutier et al. (2006) and Ladouceur and Sevigny's (2003) studies above. Both Jardin and Wulfert $(2009,2012)$ did not report the duration pop-ups appeared on screen for, so could not be compared to those found to be effective. This indicated the break element alone can have unintended effects which undermine the harm minimisation goal if the breaks are too long.

New Zealand pop-ups appear at least every 30 minutes and are displayed on screen for a minimum of 15 seconds unless the player exits the game and cashes out. These findings suggest that New Zealand pop-ups are likely to be of benefit due to the break they provide, as there is enough time between pop-ups for a state of dissociation to be entered into, and they are on screen for a relatively small amount of time compared to those that induced craving.

\section{Overview of the Current Study}

To summarise, New Zealand information displays present feedback, provide a break in play and include elements that facilitate self-appraisal. Despite the previous literature giving indications about the relative effectiveness of all these elements, we do not know whether the particular combination and timing arrangement achieves its harm 
minimisation function. Therefore, the current study was needed to evaluate the utility of New Zealand information displays.

The current thesis aimed to investigate both types of information displays in New Zealand. All four studies in this thesis carried out analyses of a dataset collected by the Department of Internal Affairs tracking New Zealand wide EGM gambling. The first two studies investigated pop-up information displays. This was conducted using a number of different methods to gain the maximum understanding of how these displays function within the New Zealand context. We investigated questions including how often the pop-ups resulted in quitting, trends surrounding their appearance and factors influencing their effectiveness as a harm minimisation tool. The last two studies investigated elective information displays which will be introduced in more detail within these studies. A range of statistical tests were employed to examine their usage and explore factors that could influence their usage by gamblers. All four studies used an exploratory investigation approach using the data to provide evidence on how information displays in New Zealand function without any preconceived ideas. This was important as New Zealand information displays had never been investigated in this way before and this dataset had never been analysed for research purposes prior to this study. 


\section{Chapter 2: Data Acquisition and Filtering}

The first aspect of this study was acquiring the dataset used for the subsequent analyses. Once acquired we intended to assess whether the data were of sufficient quality to analyse further and apply filtering methods to remove errors within the dataset resulting from technical issues. Given these data were collected by an external source and had never been examined for research purposes, there was potential for unknown technical errors to have occurred leading to errors being recorded into the dataset. Data errors could have been due to failures with how the EGM was programmed (e.g. not displaying a scheduled pop-up), within the EGM recording (e.g. not recording a pop-up after it was displayed), or during the transmission of information to Electronic Monitoring System (EMS) for collection. While it was possible to identify errors in the data it was not possible to determine at what stage the error had occurred. Identifying and removing erroneous data allowed greater confidence in results and conclusions drawn.

\section{Method}

We gained access to a large dataset collected by the New Zealand Department of Internal Affairs (DIA) tracking EGM play in New Zealand. In New Zealand, all EGMs legally must report information regarding use to a central database through an EMS. This system was set up and is currently used as a regulatory tool for the purposes of assessing compliance with banking of profits and other regulatory functions within venues. However, the information collected is also of potential value to research. Ethics approval for this study was granted by the Victoria University School of Psychology Ethics Committee. 
The process of gaining access to these data was complex. This included: approaching DIA to gain information about what data they had available; organising and attending a series of meetings to understand what was possible to investigate with the available data; writing a proposal of intended study for DIA (see Appendix A); and negotiating terms of the data-sharing agreement to reach an agreement that suited both parties (see Appendix B). Once agreement was reached and the data-sharing contract was signed it was agreed we would receive the complete set of relevant event data for a seven-day period Monday the 9th of April through to Sunday the 15th of April, 2018. We chose this week because it was the most recent week following the data-sharing approval that did not have any New Zealand wide events such as public holidays or school holidays that could influence venue opening hours or people's gambling. This resulted in data describing 1,111,189 sessions from 1,146 gambling venues including all five manufacturers.

\section{Specifics of the data}

According to the Gambling Act (2003), any EGMs that run on QCOM version 1.6.3 technology (8,840 machines in the current study) are legally required to record certain play events occurring on the machines. There were 6,604 machines in New Zealand that did not yet run on this technology therefore gambling data from these machines was not collected or analysed. While there were a number of play events recorded, two of these events were of key interest to the current study. The first event this study examined, related to pop-up displays and was labelled "EGM PID (player information display) session stopped". This event indicated the end of a person's gambling session on a particular machine. As specified in the gaming machine regulations, sessions begin when a player places a bet, and end under any of the following circumstances: 
a) a player stops playing and a period of 60 seconds has elapsed since the end of the last completed game;

b) no further play is possible without additional credits being purchased by the player and 30 seconds has elapsed since the last completed game;

c) a player has exited the game by pressing the 'collect' button on the machine or

d) a player selects the quit option on the pop-up player information display.

There is a set of information captured with this "EGM PID session stopped" event. This consisted of session information relating to: the date and time; the length; the number of pop-up information displays that were displayed; the amount the player spent; the player's net win or loss when the session ended; and how the session ended whether the player stopped the session by clicking 'no' in response to being asked whether they wish to continue play on a pop-up screen, or whether they stopped at any other time (i.e. a), b), and c) in the list above). Note that timing of individual pop-up displays is not recorded or reported in these data. It was not possible to identify individuals within this dataset therefore the same individual could contribute more than one session to the dataset.

\section{Results and Discussion}

Once the dataset was received the first check we ran to confirm data quality was calculating the return to player (RTP) value for the week. RTP is an average rate at which a machine pays money out, as a percentage of the money fed into it. It is always below $100 \%$ as on average the machines take more money in than they pay out to the players (machines make a profit). We then compared this to the RTP values required by 
the regulations of between $78 \%$ and $92 \%$. At the time of the study, most machines RTP values were set at $91.5 \%$ (True \& Cheer, 2018).

We calculated the RTP rate for the week. First, we calculated the total money won (summed all sessions' total spend and subtracted the sum of all sessions' net loss). We then divided this total money won value by the total spend value getting an RTP rate for the week of $89.79 \%$. This indicated that the data were consistent with what we should expect based on the regulations. As it is up to the manufacturers to interpret the regulations we calculated the RTP rate for each manufacturer individually. Aristocrat machines had an RTP of $88.67 \%$, AGT had an RTP of $90.35 \%$, IGT had an RTP 91.23\%, Star Games had an RTP of $91.54 \%$ and Konami had an RTP of $85.78 \%$. These were all within the range provided by the regulations. Note that each manufacturer produces many different games and while these RTPs are reflective of the average RTP for sessions played on machines produced by each manufacturer during the week, it may not be identical to the average RTP for any one individual game. There are known differences in the predetermined RTP set for each type of game, even between games produced by the same manufacturer. The RTP information for each game is available to players through the elective information displays.

The first filter we used to ensure errors were removed was identifying any potential miss-measures of session length. There were two ways to determine session duration from the data. The start of sessions were time stamped with a 'start event' and the end of sessions were time stamped with an 'end event' (specified earlier). The difference between these gave a measure of session length. For each session, there was also a variable that independently recorded the duration of the session. The time difference (stop-start) values and the recorded duration were then compared. Sessions where there was a discrepancy of greater than one minute, were removed from the 
analysis. One minute was used as the cut off for this discrepancy due to the possibility of rounding errors producing small differences. The time difference value was accurate to the nearest second while the duration value was only accurate to the nearest whole minute. This removed 3,920 sessions from the analysis. For the purposes of accuracy, the time difference value was used in the rest of the analysis as this was measured to the nearest second.

The second filtering method was removing sessions that recorded numbers of pop-ups inconsistent with the requirements of the Gambling Act (2003). The specific regulation of interest was around the timing between pop-ups. The regulations stipulated that people cannot play continuously for longer than 30 minutes without a pop-up appearing. Therefore, sessions that were longer than 30 minutes and recorded zero pop-ups, longer than 60 minutes and recorded fewer than two pop-ups, longer than 90 minutes and recorded fewer than three pop-ups, so on and so forth, were removed. We could not determine from the data provided whether these inaccuracies occurred because the machine did not display pop-ups as required, or whether they were displayed but not recorded. This removed an additional 3,126 sessions.

We also observed a further 117 sessions which recorded players quitting on a pop-up while also recording that no pop-ups occurred during the session. Because it was unclear whether this inconsistency was due to a pop-up recording failure or whether the error was in recording how the person quit, we also removed these sessions from the analysis.

The final filtering method removed sessions with a rate of play too high or too low to be possible. The maximum bet per spin in New Zealand is $\$ 2.50$. The length of each spin varies with machine, but it is unlikely people could make more than one spin 
every 3 seconds (20 spins per minute). The maximum rate of spending was therefore approximately $\$ 50$ per minute. Given this, we removed sessions with a rate of play greater than $\$ 60$ per minute. This removed 366 sessions. In addition, we removed sessions where rate of play was too small. Given the availability of a pause function where players can reserve the machine for 3 minutes at a time (which counts towards the total duration of the sessions), it was more difficult to determine a threshold for rates too low to be real sessions. Because the minimum bet per spin is 1 cent, we decided to use this as the lowest acceptable rate of play per minute. Removing sessions with less than 1 cent per minute removed an additional 186 sessions.

After all four filters were applied we had removed a total of 7,715 sessions out of the original $1,111,189$, this was removal of $0.69 \%$ of all sessions. Following this filtering there were 1,059 venues and 8,840 EGMs included in the dataset. 


\section{Chapter 3: Study 1}

Study 1 investigated machine characteristics and gambling behaviour on EGMs across New Zealand. There has been little quantitative research in New Zealand using EGM session data, so before proceeding to investigate the specifics of how pop-ups functioned, it was important to look at how EGM gambling in New Zealand was conducted. We asked a number of key questions.

The first question was: what are the characteristics of an average session? This was to gain an understanding of whether this dataset was consistent with what we would expect gambling in New Zealand to be, given other available information.

The second question was: how frequently do people use pop-up information displays to exit games? Answering this question had two key purposes. The first was to determine whether the number of sessions on which gamblers quit on a pop-up was sufficient to compare the features of those sessions with sessions that did not end on a pop-up. The second purpose was to use this number as an indication of how effective pop-ups were as a harm minimisation intervention.

The third question on pop-up displays was: how many pop-ups occur and how are they distributed? Answering this question also had two purposes: firstly, to determine which further analyses were possible to conduct on the dataset, and secondly, to better understand how different manufacturers have interpreted the legislation.

The last question was: are quitting rates altered in the anticipation of a pop-up or following their appearance? The aim of this was to determine whether pop-ups had an influence on quitting, apart from an immediate response while they are displayed on the screen. 


\section{Method}

All calculations were completed using the filtered dataset. Rstudio software, version 1.0.153 was used to conduct all analyses on the dataset.

\section{Results and Discussion}

\section{Play in New Zealand}

We calculated descriptive statistics for session lengths, spends, net wins/losses and rates of play. These are shown in Table 3.1. Note the means are consistently more extreme than the medians due to a skewed distribution.

Table 3.1

Gambling session descriptive statistics

\begin{tabular}{lccccc}
\hline & Median & Mean & $\begin{array}{c}\text { Standard } \\
\text { Deviation }\end{array}$ & Minimum & Maximum \\
\cline { 2 - 5 } Session length & 4.68 & 9.93 & 16.43 & 0.03 & 694.72 \\
(minutes) & & & & & \\
Session spend & 36.60 & 111.77 & 269.07 & 0.01 & 15302.06 \\
(dollars) & & & & & \\
Net Win/Loss & -10.12 & -11.41 & 87.27 & -3200.00 & 2330.12 \\
(dollars) & & & & & \\
Rate of play & 7.22 & 10.09 & 8.18 & 0.01 & 58.57 \\
(dollars/minute) & & & & & \\
\hline
\end{tabular}

Players spent a mean of close to 10 minutes playing continuously at one machine with a median of 4.68 minutes. This finding was useful as our study examined 
the potential effects of pop-up displays, which were required to appear prior to 30 minutes of continuous play at one machine. One aim of harm minimisation interventions is to specifically target people experiencing harm, rather than those who are playing recreationally (Gainsbury \& Blaszczynski, 2012). Given the average time of play was below the required threshold for seeing a pop-up, it is possible that the average player does not tend to see a pop-up during their session. This suggested that pop-ups could be targeting those playing for longer than average, who are more likely to be associated with harmful gambling, rather than targeting all gambling sessions.

The mean player spend per session was $\$ 111.17$, with a median of $\$ 36.60$. While this mean value could appear high it is important to note that 'spend' includes not only the credits played as a result of players feeding money into the EGM, but also as a result of spending credit they have won back on spins. Spend was therefore not a reflection of how much the player expended of their own money initially, but a reflection of how many credits were wagered on the machine. Data on money fed into machine initially was not available in the dataset.

In an example using an exact RTP of $90 \%$ occurring on every spin, a person could bet $\$ 2.00$ on a spin and win $\$ 1.80$ back (this constant RTP is used for ease of understanding in the example, however as it is an average value, this could only occur on average, in reality each spin has a random outcome). They could then spend the $\$ 1.80$ they won on another spin bringing their total spend to $\$ 3.80$. If they won $\$ 1.62$ back on that spin, they could then spend these winnings on another spin, so on and so forth. In just the three spins their total spend would be $\$ 5.42$ while they still had initially fed $\$ 2.00$ into the machine. In just seven spins the player could end up with a total spend of $\$ 10.45$ while still only initially using $\$ 2.00$. Taking this into account a mean 
spend per session of either $\$ 111.77$ or $\$ 36.60$ was a plausible value given that the mean session length was around 10 minutes and median duration was 4.68 minutes.

The mean rate of play per session was just over $\$ 10$ per minute. This was another way of representing the spend and session length statistics. Rate of play is independent of the session length so was useful for comparing across different sessions. This measure was used in Study 2.

The mean net loss value to players was $\$-11.41$. Given that this was the amount lost by the players, and gained by the gaming industry, this negative value per session reflected the house winning. This value was consistent with annual gambling expenditure statistics. With approximately $1,103,474$ sessions occurring per week (on the machines included in the dataset) and player expenditure of just over $\$ 10$ per session, this resulted in an overall weekly player expenditure of $\$ 12,586,443$. Across the year this would be an expenditure of $\$ 654,495,083$. According to the annual statistics, total spending on EGMs in New Zealand was \$870,000,000 in 2017 (Department of Internal Affairs, 2017). Given the number produced by the Department of Internal Affairs included all the machines, and there were 6,580 machines not included in the current dataset because they ran on technology older than QCOM version 1.6.3, the value produced from the dataset was within the expected range.

\section{Frequency of Pop-ups Resulting in Quitting}

The second question Study 1 answered, was: how often were pop-ups used to quit games? Of the $1,103,474$ sessions 3,875 ended because the player quit on a pop-up. The first purpose of answering this question was to check whether the number of popups resulting in a player quitting the game would be high enough to conduct further analyses. We were confident that the 3,875 sessions would give us the statistical power 
to detect effects that were present (power analyses were not conducted at this stage as they differ for each statistical test).

The second purpose of this question was to use the frequency with which people quit a gambling session on a pop-up as an indication of how effective pop-ups are as a harm minimisation intervention. To begin to answer this question we observed that $0.35 \%$ of all sessions ended on a pop-up. As not all sessions showed pop-ups this figure was also calculated as a percentage of the sessions that displayed at least one pop-up, increasing the percentage to $3.13 \%$. Because some sessions showed multiple pop-ups, the total number of pop-ups resulting in quitting as a percentage of the number of popups appearing during the week was $1.93 \%$. All percentages indicated this harm minimisation tool being used at very low frequencies.

Literature and policy documents did not provide clear guidelines to determine what frequency of quitting on a pop-up would be sufficient to conclude pop-ups were effective harm minimisation tools. We however, considered it unlikely for a tool designed to help people quit, to be working effectively when it resulted in quitting on fewer than $2 \%$ of presentations. Pop-ups aim to help people to gamble more responsibly by providing information and prompting an active choice about whether they should continue gambling or not. The interpretation of observed rates of quitting was therefore complicated by the fact that not all people who gamble on EGMs experience harm from them. There are a group of people who gamble recreationally, within their means. For these individuals, responding to the question 'do you wish to continue playing' with a 'yes' response, is a responsible gambling decision for them. This means that even if these pop-ups were as effective as they possibly could be, we would not expect a quitting rate of $100 \%$. However, if they were working as best as they could, we would expect a quitting rate higher than those observed in our dataset. 
One reason we would have expected this number to be higher if pop-up displays were working effectively was that $43 \%$ of regular EGM players fitted the classification for problem gambling, or were in a moderate risk group, making them the target population for this harm minimisation tool (Gray, 2011). Even if every pop-up that resulted in quitting was from a person in this risky group (giving the most inflated value), this would at the most be $4.65 \%$ of sessions from the target group using pop-ups to quit. This further supports the idea that the tool is not working as well as it could be.

Palmer du Preez et al. (2014) also looked at the rate at which a pop-ups resulted in quitting in their study of New Zealand EGMs. They found that on four pop-ups (22\%) out of the 18 pop-ups observed, players cashed out when they saw a pop-up. This percentage was higher than the findings of the current study indicating that there could have been differences between the studies that led to pop-ups being used at different rates. However, given that their study had such a small sample size (18) it was possible this did not reflect a meaningful difference. The reliability of a population estimate based on a small sample is limited. It was likely that our study was able to refine the overestimation in Palmer du Preez et al.'s study.

\section{Distribution of pop-ups}

The next question we answered in Study 1 was: how many pop-ups occurred during the week and how were they distributed? Across the week 200,734 pop-ups occurred during the EGM play sessions. The first way we looked at distribution was by breaking up the occurrence of pop-ups by each day of the week, seen in Table 3.2.

Rate of play varied throughout the week, with greater numbers of sessions on Friday and Saturday and lower play on Sunday and Monday as would be expected based on previous observations (Kairouz, Paradis, \& Monson, 2015). It does, however, 
remain unexplained that Thursday displays the second highest number of sessions played. Anecdotally, it is possible that Wednesday night is a common payday in New Zealand, but we could not verify this.

While the number of gambling sessions and pop-ups seen varied across the week, the proportion of pop-ups resulting in quitting was strikingly stable. A chisquared test of independence indicated no significant effect of day, on the percentage of pop-ups resulting in quitting $X^{2}(6, N=200734)=6.05, p=.417$.

Table 3.2.

Pop-up sessions by days of the week

Monday Tuesday Wednesday Thursday Friday Saturday Sunday

\begin{tabular}{llllllll}
\cline { 2 - 6 } Total sessions & 109,363 & 147,220 & 176,078 & 184,294 & 191,733 & 177,041 & 117,745
\end{tabular}

Sessions with

$\begin{array}{lllllll}11,772 & 15,554 & 20,420 & 21,554 & 22,415 & 19,603 & 12,474\end{array}$

pop-ups

Total number

$\begin{array}{lllllll}18,785 & 25,254 & 33,244 & 35,545 & 36,691 & 31,479 & 19,745\end{array}$

of pop-ups

Total pop-ups

$\begin{array}{llllllll}\text { resulting in } & 365 & 472 & 657 & 678 & 666 & 642 & 395\end{array}$

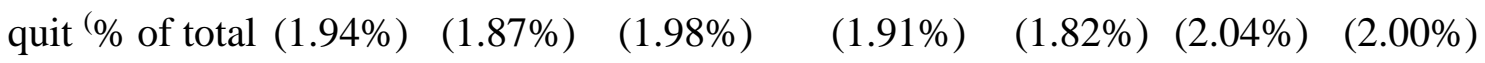

pop-ups seen)

As stated in the introduction, manufacturers could interpret regulations surrounding pop-up implementation differently. For this reason, it was useful to look at 
the distribution of pop-ups, and their rate of use, broken up by the five manufacturers: AGT, Aristocrat, IGT, Konami, and Star Games. Table 3.3 shows that there is not only large variability in the total sessions played on machines produced by different manufacturers, but also large variability in the percentage of pop-ups resulting in a player quitting across the manufacturers. A chi-squared test of independence indicated a significant relationship between manufacturer and the percentage of pop-ups resulting in quitting $X^{2}(4, N=200734)=1484.2, p<.001$. Post hoc tests revealed that all pairwise comparisons were significant (adjusted using the false discovery rate method). While these post hoc comparisons were all significant, their effect sizes were small (ranging from 0.005- 0.095). The largest three effect sizes (0.07-0.095) were for the differences between AGT and both: IGT and Star Games, and between Aristocrat and Star Games. 
Table 3.3

Pop-up sessions by manufacturers

\begin{tabular}{llllll}
\hline & AGT & Aristocrat & IGT & Konami & Star Games \\
\cline { 2 - 6 } Total sessions & 179,668 & 383,631 & 217,699 & 98,081 & 224,395 \\
Sessions with & 26,767 & 44,668 & 21,113 & 4,870 & 26,374 \\
pop-ups & & & & & \\
Total number of & 43,728 & 75,098 & 31,745 & 7,075 & 43,097 \\
pop-ups seen & & & & & \\
Total pop-ups & & & & & \\
resulting in quit & 407 & 791 & 912 & 126 & $(3.80 \%)$ \\
(\% of total pop- & $(0.93 \%)$ & $(1.05 \%)$ & $(2.87 \%)$ & $(1.78 \%)$ & \\
ups seen) & & & & & \\
\hline
\end{tabular}

This finding indicated players' response to pop-ups could be influenced by manufacturer-specific features of the pop-ups. At this point however, it remained unclear whether the apparent relation between manufacturer and response to pop-ups would remain when accounting for other available variables. We assessed this in Study 2.

Following evidence that rate of quitting on a pop-up differed across the manufacturers, the next step was examining the distribution of pop-ups to uncover potential reasons. Figure 3.1 presents the distribution of sessions containing at least one pop-up presented for each manufacturer. This indicated different manufacturers had 
interpreted the regulations about the timing of pop-ups differently and scheduled pop-up onset times in different ways.

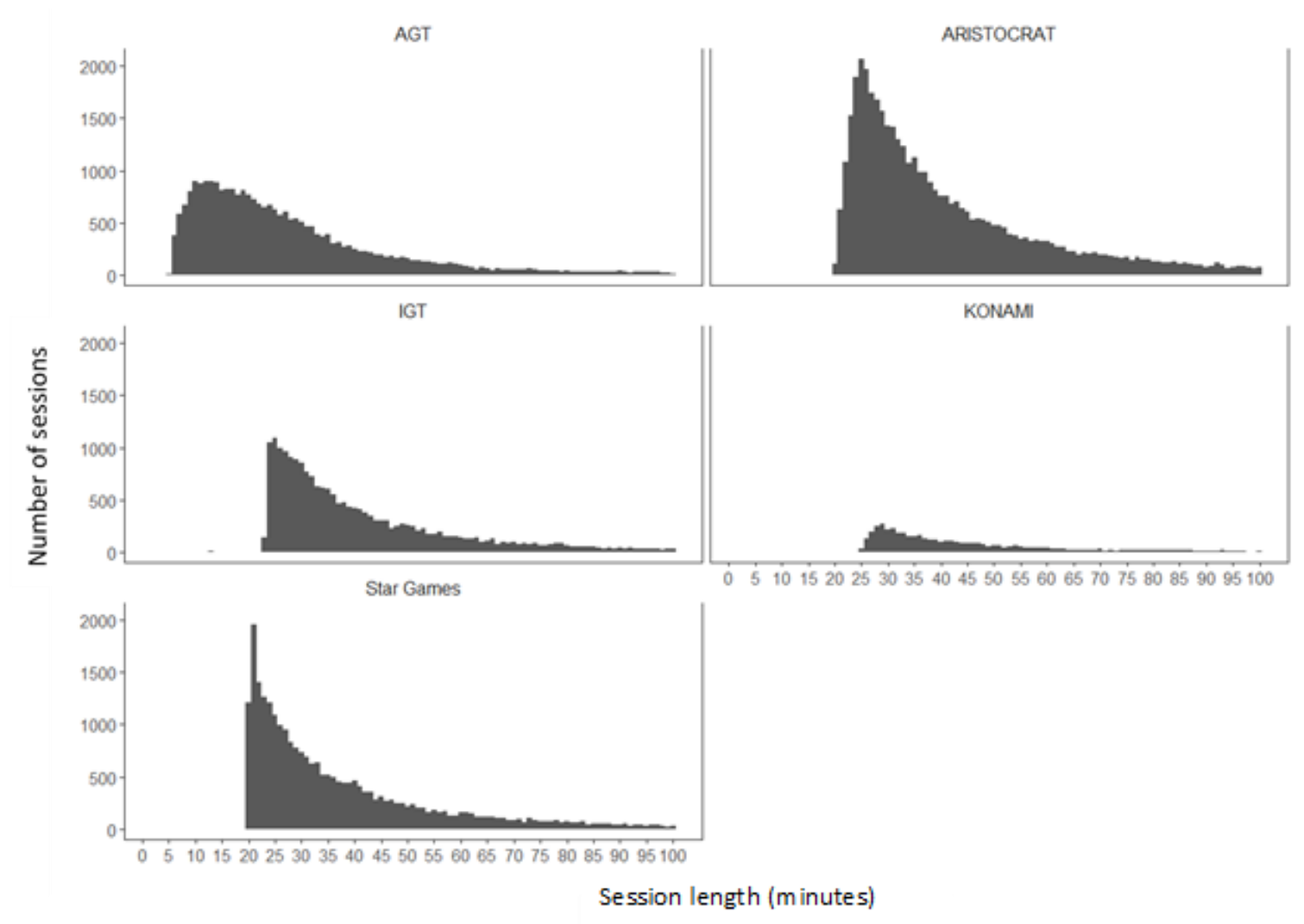

Figure 3.1. Histograms of session length where at least one pop-up occurred by each of the five manufacturers.

Regulations specify that pop-ups must occur randomly in a set period of time no less than 5 minutes, which must occur prior to 30 minutes passing in a session (see wording of regulation above; Department of Internal Affairs, 2016). This wording gives the manufacturers freedom to decide when the first pop-up will appear, anywhere between 0 and 30 minutes into the session.

The dataset did not include the times pop-ups appeared. To estimate when the earliest pop-ups occurred for each manufacturer, we identified the shortest sessions in the dataset where at least one pop-up had appeared. This is shown in Figure 3.1. For AGT there were 0 sessions with a pop-up appearance ending between 4 and 5 minutes, 
however there were 378 sessions with a pop-up appearance ending between 5 and 6 minutes. This was a good indicator that 5 minutes was the minimum pop-up onset time for machines manufactured by AGT. From Figure 3.1, we can also see that the minimal onset pop-up time was 20 minutes for machines manufactured by Aristocrat, 23 minutes for machines manufactured by IGT, 25 minutes for machines manufactured by Konami and 20 minutes for machines manufactured by Star Games. This suggested the presentation of these harm minimisation tools is different across manufacturers. For players gambling for a shorter duration than the average session, the likelihood of seeing a pop-up, depended on which manufacturer made the machine they are gambling on.

The next logical question to ask was whether the differences in pop-up quitting rate across manufacturers seen above, were driven by the differences in onset time. If people simply became more and more likely to quit over time, then manufacturers with earlier onset times for their pop-ups would have fewer instances of people quitting on a pop-up, as people may not intend to leave so soon after they started play.

Figure 3.2 shows the quitting on a pop-up percentages for each manufacturer in order of onset time, lowest to highest. Pop-up onset times are overlaid onto each manufacturer's bar. A systematic relationship between onset time and quitting rate would be indicated by a monotonic change in bar height. There is no systematic relationship between pop-up onset time and rate of quitting on pop-ups. Note that Aristocrat and Star games, both have an onset time of 20 minutes, but their rates of quitting are strikingly different. While it is unclear to what extent onset times impact quitting rate, alone they are not sufficient to explain the differences found in quitting percentages between the manufacturers. 


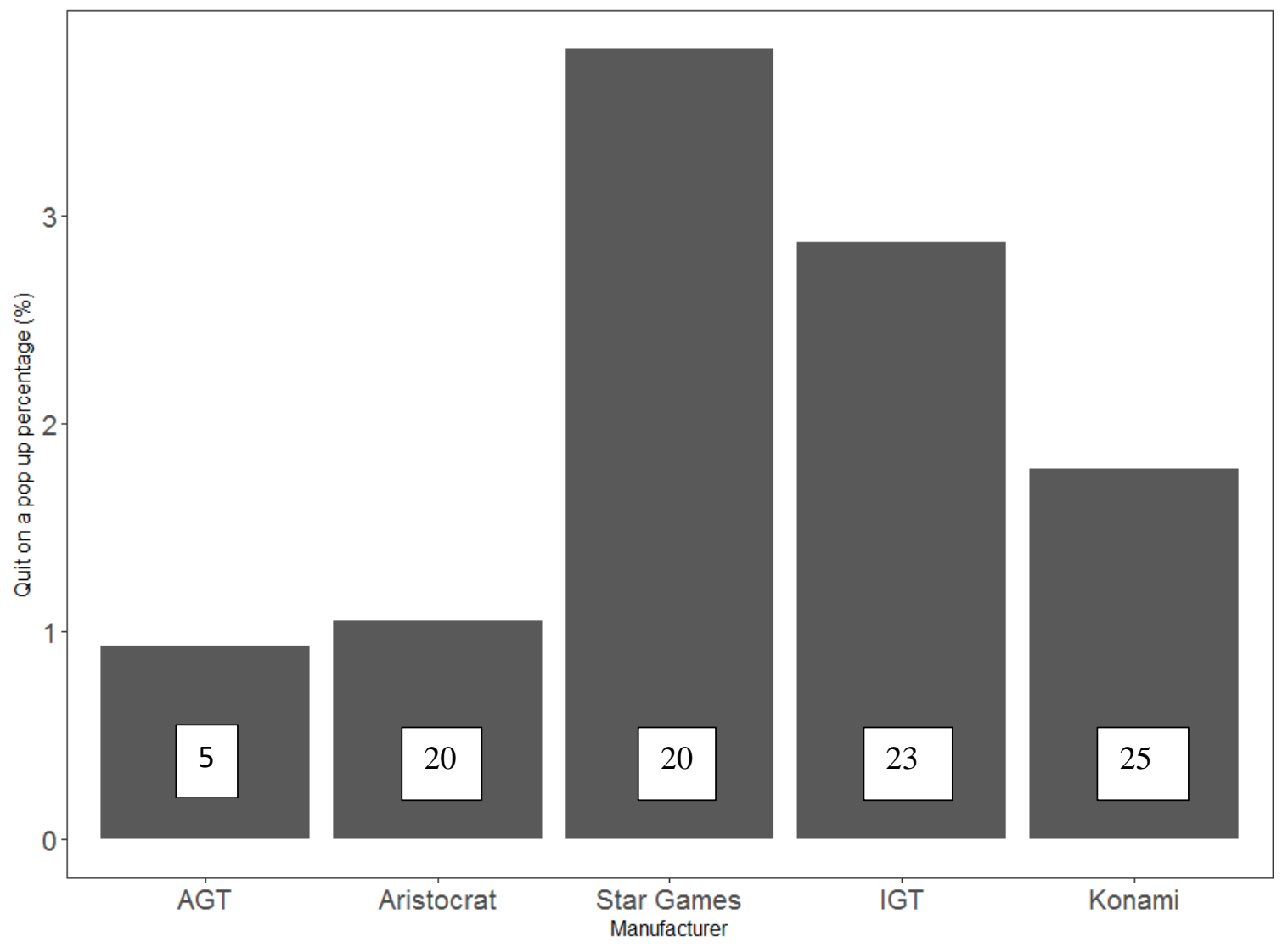

Figure 3.2. Quit on pop-up rates with pop-up onset times for manufacturers' overlaid.

Given all manufacturers have different onset times but still meet the regulations, we investigated other ways pop-ups could differ between manufacturers, while still complying with the regulations. The distributions of sessions with pop-ups separated by manufacturer, revealed further ways in which pop-ups differed. Figure 3.3 shows all sessions that ended on a pop-up, across the five manufacturers. While this figure includes fewer sessions than Figure 3.1, it provides information on the exact times that pop-ups occurred. The end of session time stamp for sessions that ended by quitting on a pop-up also record accurate timing information about pop-up appearance. Figure 3.3 displays clear differences in the times pop-ups tended to appear. AGT and Aristocrat machines have pop-ups that appear at random times, making them unpredictable. However, on IGT, Konami and Star Games machines, pop-ups appear in clusters. For IGT this can be seen between 23-35, between 45-60 and between 70-75 minutes. For 
Konami this can be see between $25-30$, between $50-60$ and between $80-85$ minutes. For star games this can be seen between 20-25, between 40-45, between 60-65 and between 80-85 minutes.

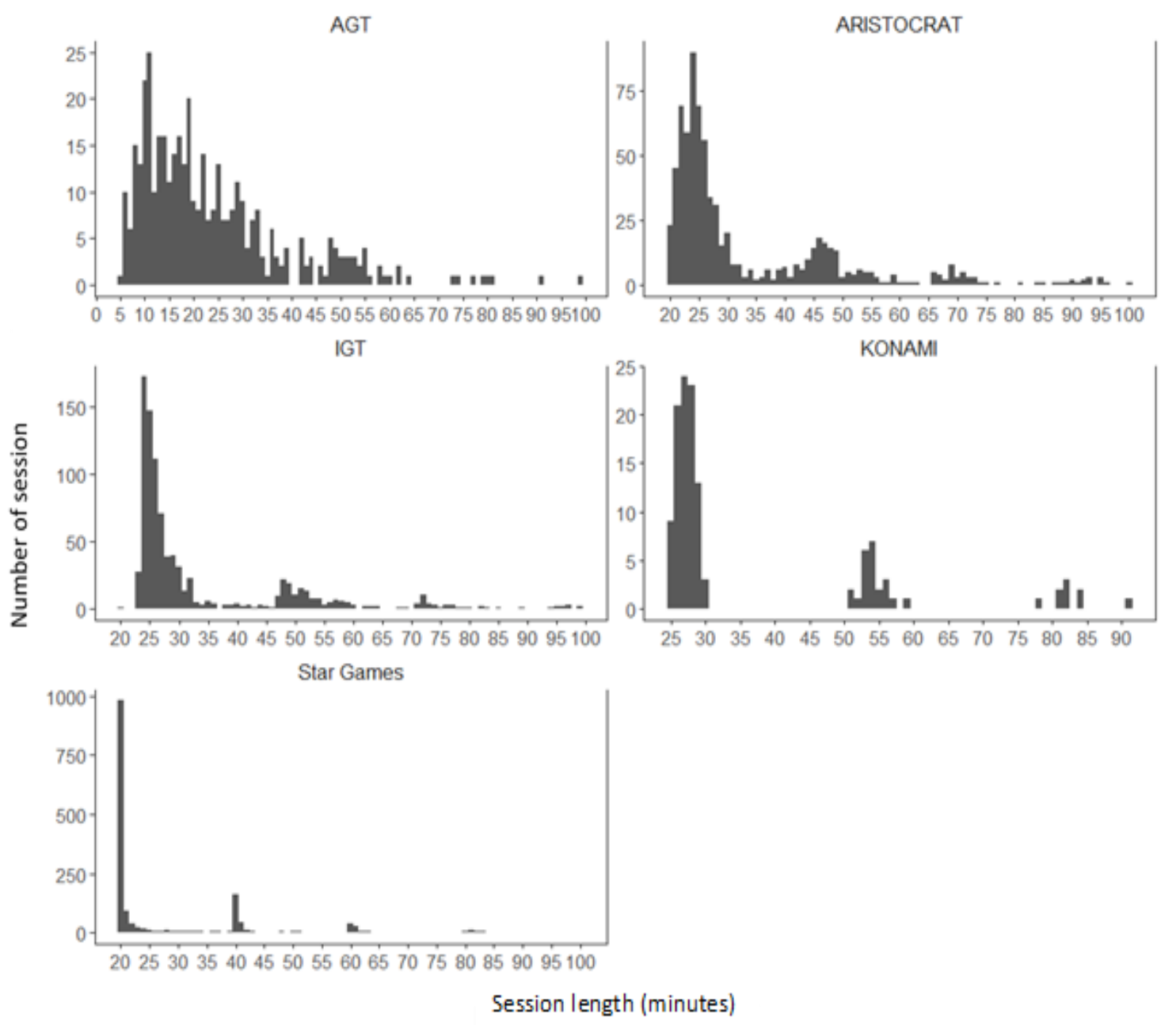

Figure 3.3. Histograms of session length where players quit on a pop-up by each of the five manufacturers. Note that $y$-axis scales are customised by manufacturer.

These differences are due to differences in the programming of pop-up scheduling throughout the session and not just a flow-on effect of the onset times. This is apparent because Aristocrat and Star Games, which have the same onset time, display different pop-up timing patterns.

Making pop-ups appear at predictable times could influence how well they work at minimising harm. There is evidence from focus group literature suggesting players 
dislike pop-ups appearing. Some feel displaying information about sessions on the screen for others to see is a breach of privacy (Palmer du Preez et al., 2014). Having predictable pop-ups could encourage gamblers to prevent their appearance by periodically ending sessions by cashing out. They could then reinsert their money in the same or a different machine to begin a new session on which a pop-up would not occur for many minutes. Following this, it is interesting a trend can be observed where manufacturers with the most systematic clustering are those that have the highest rates of quitting on a pop-up. This suggests predictable pop-up timing improves the utilization rate, contrary to the idea presented above.

\section{Distal effects of pop-ups}

While gamblers rarely quit using a pop-up display, it is possible that their presence has a more distal effect on encouraging gamblers to quit. If people dislike seeing pop-ups and can predict when these will appear, we might predict a spike in quitting just prior to the time pop-ups are programmed to appear. It is also possible and is suggested by Palmer du Preez et al. (2014) that following the appearance of a pop-up, people may play a few more spins to finish their credit, and then end their session. This quitting however is still prompted by the pop-up appearing. Within the current dataset we could not determine how soon after a pop-up each session ended. We could however, examine quitting rates in the period before, during and, after, the time at which pop-ups most frequently occurred for any given manufacturer. Spikes in quitting can be seen by an increased occurrence of sessions ending at a particular time on a histogram of session lengths. There is a general decrease in number of sessions over time, so spikes would stand out as not fitting this general downwards trend. Figure 3.4 shows all session lengths for AGT and Aristocrat machines. Both AGT and Aristocrat have unpredictable pop-ups that are displayed at random times as can be seen on Figure 
3.3. Both figures show a smooth trend downwards with no obvious spikes or dips indicating that there are no times in which there is greater or less quitting than would be expected as session length increases.

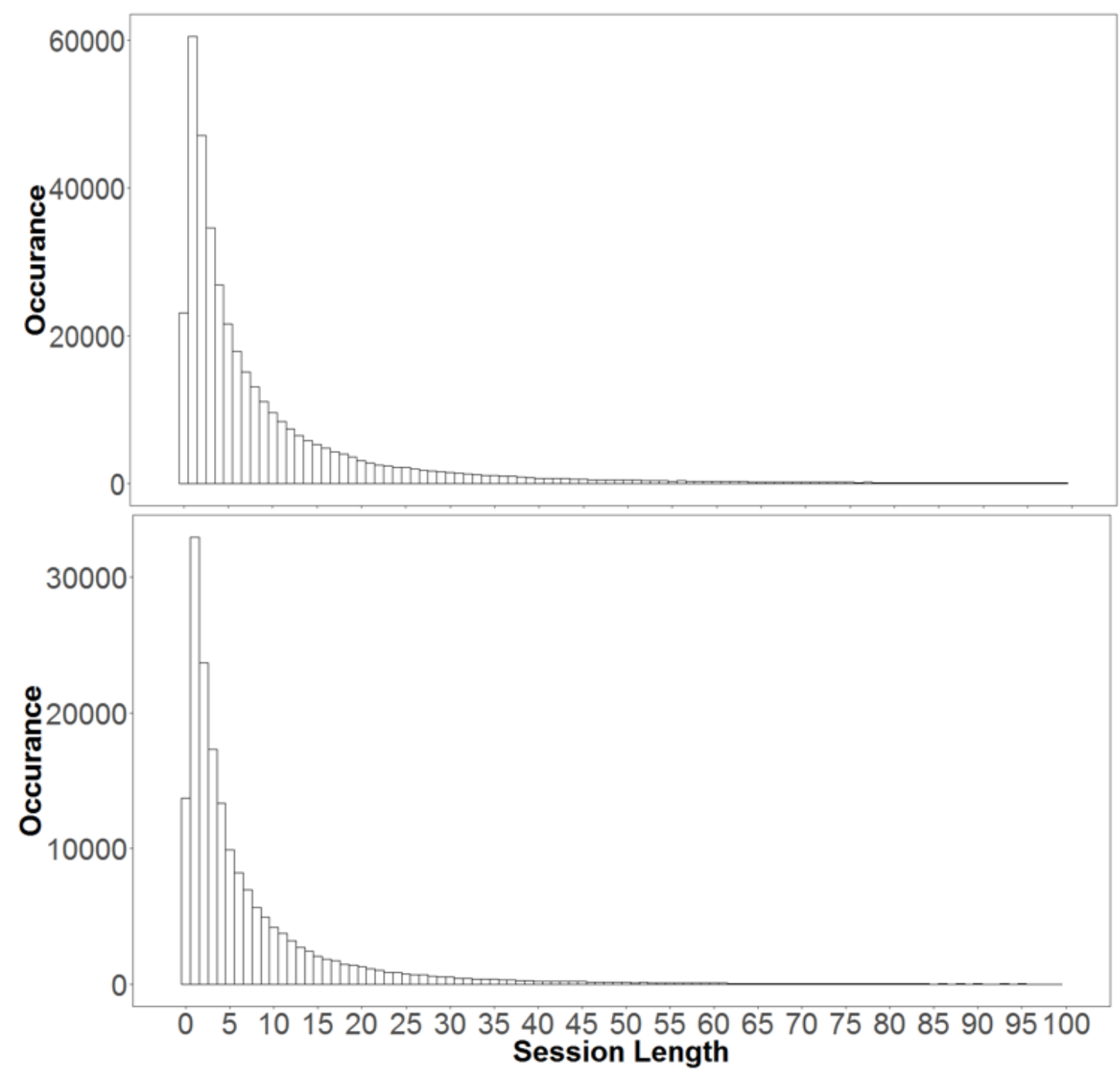

Figure 3.4. Histogram of all session lengths for AGT machines (top) and Aristocrat (bottom). Note. $y$-axes display different scales.

Figure 3.5 shows all session lengths for IGT. On first appearance there is an expected smooth downwards trend in the rate of quitting across session lengths. As seen in Figure 3.3 pop-ups on IGT machines cluster around certain times. Figure 3.5 also shows three zoomed in sections of the top graph corresponding to session lengths surrounding pop-up clustering for IGT machines. The grey shading indicates the five 
minutes before, and five minutes after the pop-ups tended to appear as identified from Figure 3.3 above. There is no spike in quitting just prior or just following the time popups appear. There does however seem to be a small drop in quitting for sessions that are 23 minutes in length. This corresponds to pop-up onset for this manufacturer. This could be further investigated by examining session length data from Aristocrat machines in additional weeks.

Figure 3.6 displays all session lengths for Konami, as displayed for IGT in Figure 3.5. Again, there did not appear to be any change in the downwards trend of sessions ending across time, corresponding to times that pop-ups most frequently occurred. Despite the bottom left graph of Figure 3.6 revealing some fluctuation, these absolute number of occurrences were very low and only differed by about 5 sessions. This fluctuation most likely did not reflect a meaningful change in quitting.

Figure 3.7 displays all session lengths for Star Games, as displayed in the previous two figures. Similar to the other manufacturers, there did not appear to be any unexpected trends in quitting across session lengths.

There appeared to be a small spike in quitting at 21 minutes which could have been related to the pop-ups themselves, given that this fell within the time pop-ups were known to appear. This was especially possible given that Star Games is the manufacturer with the highest rate of quitting via the button on the pop-up. Other than this, there did not appear to be any other unusual trends in the downward pattern of the number of sessions ending across time. There was therefore no indication that pop-ups were affecting quitting prior or following pop-up appearance. 
From the available data there was no strong evidence to suggest that pop-ups had any distal effect on quitting. Across all the manufacturers there was no evidence of increased quitting prior or following pop-ups. 

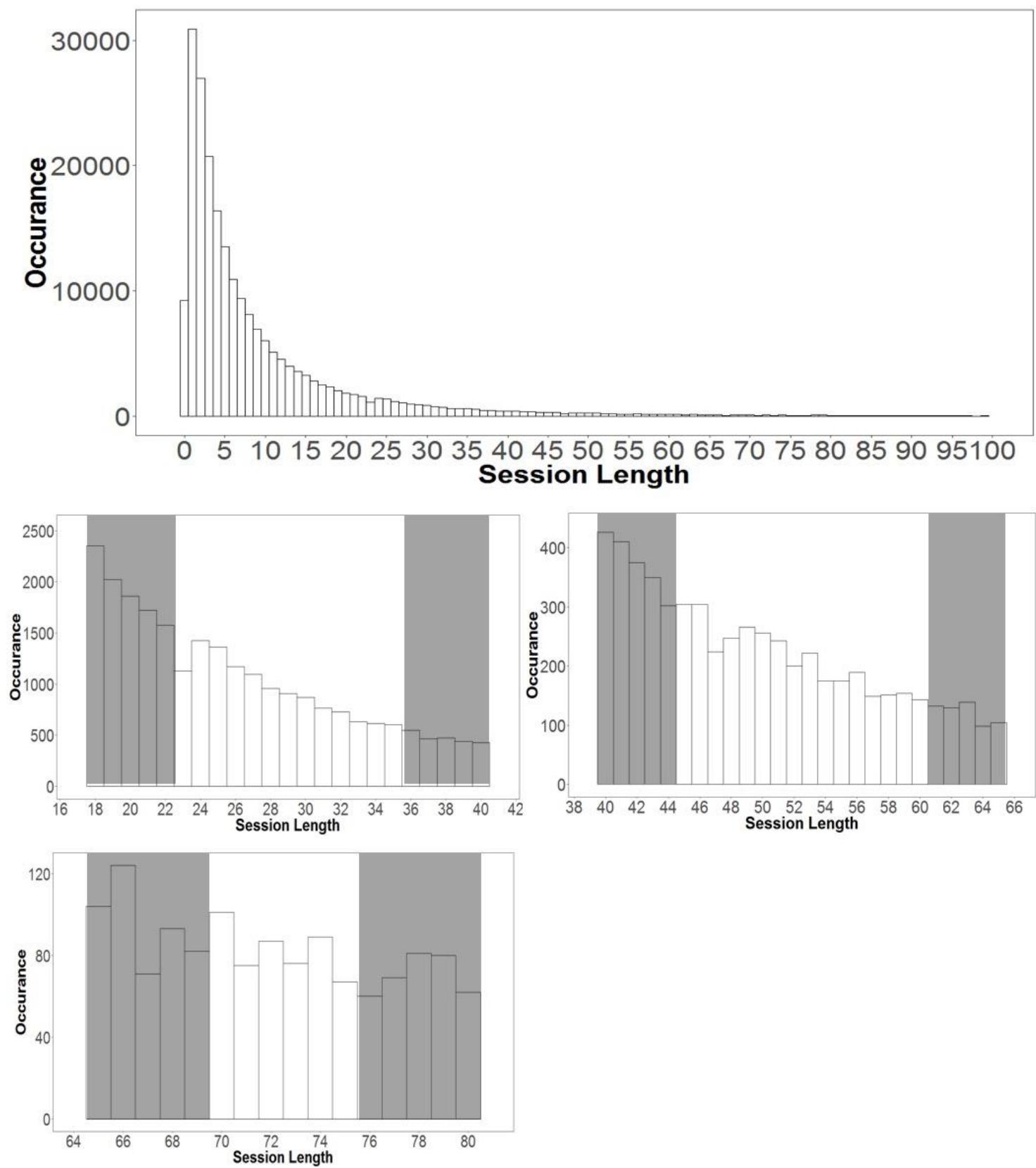

Figure 3.5. Histogram of session lengths for IGT machines. The top graph displays a histogram of all sessions. The following three graphs display a subset of the same data zoomed in to sections corresponding to session lengths where pop-ups tended to appear on IGT machines. The middle left graph shows the time span surrounding the first popup cluster, middle right shows the time span surrounding the second pop-up cluster, bottom left shows the time span surrounding the third pop-up cluster. Note $y$-axis scales differ across graphs. 

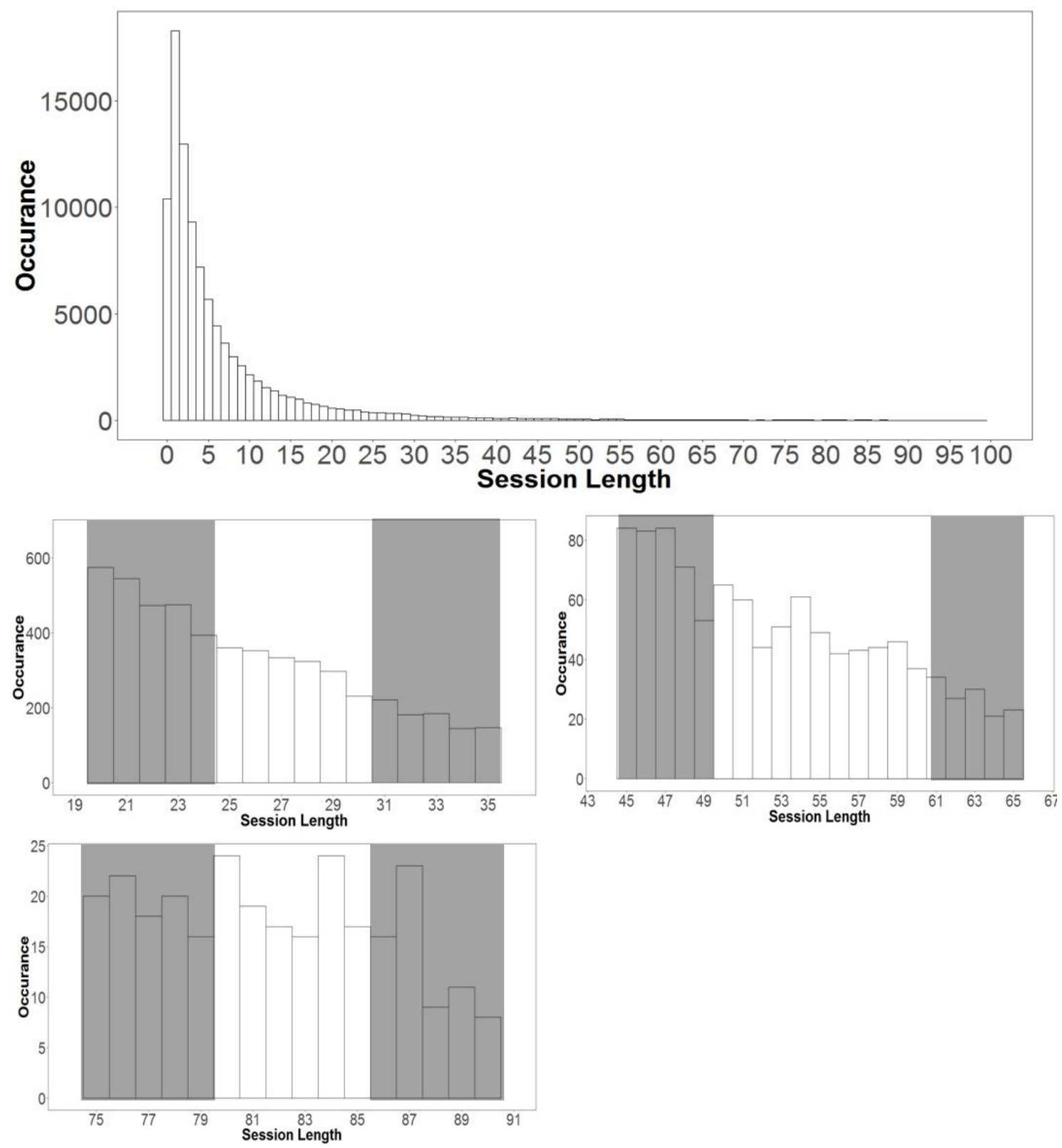

Figure 3.6. Histograms displaying session lengths for Konami machines. The top graph displays a histogram of the occurrences of sessions ending across time. The following three graphs repeat a subset of the same data zoomed in to sections corresponding to session lengths where pop-ups tended to appear on Konami machines. The middle left graph shows the time span surrounding the first pop-up cluster, middle right shows the time span surrounding the second pop-up cluster, bottom left shows the time span surrounding the third pop-up cluster. Note $y$-axis scales differ across graphs. 

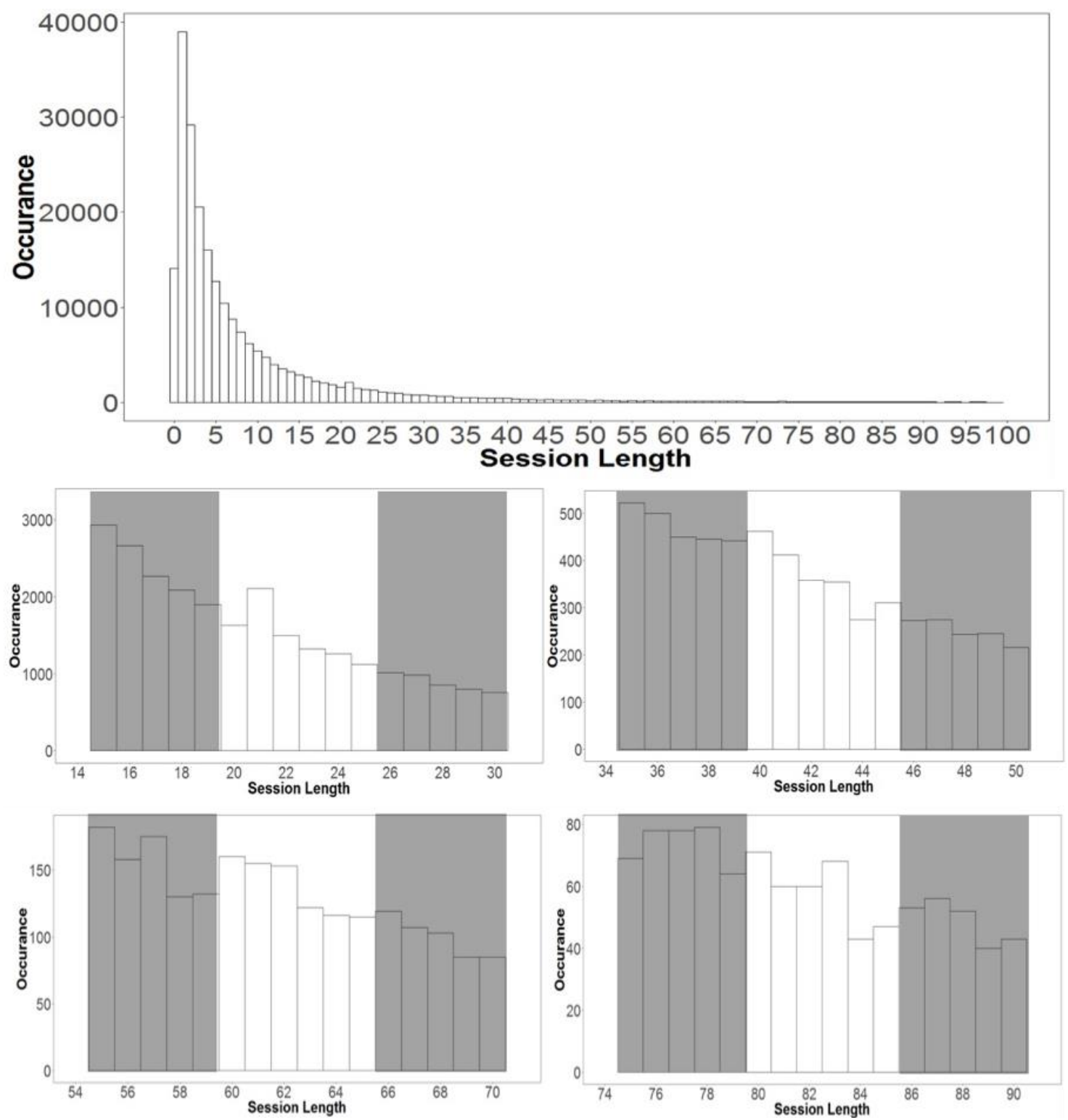

Figure 3.7. Histograms displaying session lengths for Star Games machines. The top graph is a histogram showing the occurrences of sessions ending across time. The following four graphs repeat a subset of the same data zoomed in to sections corresponding to session lengths where pop-ups tended to appear on Star Games machines. Middle left shows the time span surrounding the first pop-up cluster, middle right shows the time span surrounding the second pop-up cluster, bottom left shows the time span surrounding the third pop-up cluster, bottom right shows the time span surrounding the fourth pop-up cluster. Note $y$-axis scales differ across graphs. 


\section{Chapter 4: Study 2}

The results of Study 1 suggested pop-up information displays had a minimal impact on gambling session length. It was also clear their impact differed significantly across the manufacturers that produced them. The goal of Study 2 was to investigate whether there were any factors associated with quitting on a pop-up, when all other factors were held constant. This would allow us to determine whether the effect of manufacturer, or any other of the variables we had access to, contributed to whether or not a session ended on a pop-up. This would be a good starting point for making popups more effective at encouraging responsible gambling and increasing players' likelihood to quit sessions.

This was an exploratory study because we had no prior knowledge of which variables, if any, would be significant predictors of quitting on a pop-up. We included as many variables as we had access to. Given the findings of Study 1 , we expected that manufacturer would influence the likelihood that sessions would end via a pop-up, and that day of the week would not. However, we had not controlled for other variables in Study 1, so in Study 2 we investigated whether these findings held while controlling for all other available variables.

The question we asked in Study 2 was: what variables influence whether someone will quit on a pop-up as opposed to quitting at any other time? Given that the dependent variable was dichotomous and the predictor variables were a mix of categorical and continuous, we used logistic regression.

\section{Method}

From the filtered dataset described above, Study 2 used 123,790 sessions on which at least one pop-up occurred. Because the question that we were asking was 
specifically about quitting on a pop-up it was essential to limit our dataset to only include sessions where at least one pop-up had been seen.

\section{Selection and Categorisation of Predictor Variables}

The variables available in the dataset were as follows:

1. The day of the week

2. The venue type

3. Region

4. Suburb

5. EGM manufacturer

6. EGM model (which reflects the gambling game)

7. Time of day

8. Session length

9. Number of pop-ups seen

10. Spend during the session

From these it was possible to derive:

11. Net winning (gain/loss amount for the session)

12. Rate of play (calculated as dollars per minute)

The day of the week variable was coded as Monday to Sunday. The venue type variable had 14 levels, these were: hotel, non-commercial commercial club, noncommercial cosmopolitan club, non-commercial other sports club, non-commercial bowling club, non-commercial RSA club, non-commercial workingmen's club, other non-commercial premises, other commercial premises, pool hall, restaurant, TAB, tavern, and ten pin bowling premises. To ensure there were enough instances of each 
quitting type (on a pop-up and at any other time) in each level, we combined other noncommercial premises and other commercial premises into one 'other premises' category. We also added non-commercial bowling club to the non-commercial other sports club category. This resulted in 12 levels for venue type.

Due to the large number of levels in both the predictor variables: suburb and EGM model; there were not instances of sessions ending on a pop-up and sessions ending at other times in every level. Because these were already captured by manufacturer and region variables, and there was no natural way to re-group these variables, we did not include them in the logistic regression analysis. This exclusion was important because logistic regression requires observations in each unique combination of variable levels.

Time of day was a continuous variable, however, a more meaningful way to organise this variable was to categorise it and code it according to the typical work day. We created six time of day categories: 9am-12pm= morning, $12 \mathrm{pm}-2 \mathrm{pm}=$ lunchtime, $2 \mathrm{pm}-5 \mathrm{pm}=$ afternoon, $5 \mathrm{pm}-7 \mathrm{pm}=$ after work, $7 \mathrm{pm}-11 \mathrm{pm}=$ evening, $11 \mathrm{pm}-9 \mathrm{am}=$ overnight.

Session length, session spend, rate of play (dollars per minute), number of popups seen in session, and net wins/losses were continuous variables. We observed that the variable of net winnings had a possible non-linear relationship with quitting on a pop-up, therefore we categorised sessions as either: win (sessions where net gain was 0 or greater) and loss (sessions where net gain was less than 0 ). While these categories only captured a player's net gain/loss when the session ended, it is likely to be an indication of how their overall session went and will therefore be referred to as session win and loss throughout. 
Region was coded within the dataset as Northern, Central and Southern. The dataset did not define the boundaries of these regions. The manufacturers were the same as seen in Study 1: AGT, Aristocrat, IGT, Konami and Star Games.

\section{Data Analysis}

Given this study was exploratory, we tested the validity of our model. To achieve this, we created a training dataset containing a random $75 \%$ portion of the data. This training set was used to build our logistic regression model and to make inferences. The remaining $25 \%$ of data not used to build the model and 'unseen' by the model, was used to test the model's predictive ability and validate the findings.

Due to the exploratory nature of the study, we started with the most complex model possible and systematically removed terms that did not contribute to predicting the outcome (backwards selection). The most complex model that could be run given the number of predictor variables was a model that included all two-way interactions (including all three-way interactions exceeded the available computing ability).

We used VIF values to test the assumption of no multi-collinearity between the variables. This assumption was violated by high correlations between session length, number of pop-ups seen, and session spend. We decided the most important term to retain for the research question about pop-ups was the number of pop-ups seen, so we removed the other two variables. Not including session length and session spend had the potential limitation of omitting variables related to gambling intensity, therefore we included the variable rate of play (in dollars per minute) which is another potential measure of intensity that did not violate the assumption of multi-collinearity. 
Linearity was assessed visually for the remaining two continuous variables; rate and number of pop-ups seen. There were no obvious non-linear patterns present in the scatter graphs plotting the log odds versus the predictor values for either.

Explanation of logistic regression. Unlike ordinary regression which predicts continuous values, logistic regression creates a model to predict a binary outcome. In order to do this, a logistic model needs to predict the probability of an outcome. Probabilities however, are restricted to values between 0 and 1 which is problematic when the model itself can produce values between negative and positive infinity. To solve this and remove these bounds, the logistic regression model predicts the log of the odds rather than the probability. The transformation of probability to the odds removes the upper bound and the transformation to the log removes the lower bound. This then makes the outcome value bounds the same as the model bounds; between positive and negative infinity.

Converting between the log odds, the odds, and the probability can be done with a simple calculation, however the relationship between these different ways of expressing the same values is worth noting. For every 10 -fold increase in the odds, the $\log$ odds increase by 2.3026 . The relationship between odds and probability however is non-linear.

At more extreme values of the odds (far away from 0), a 10-fold change has little impact on the change in the probability of an outcome. However, at less extreme values (closer to 0) a 10-fold change in the odds corresponds to a large change in the probability of an outcome. These relationships between log odd, odds and probability are more easily understood with an example. A 10-fold change in the odds from 1 to 0.1 is equivalent to a change in the log odds of 2.30 , from 0 to -2.30 . This equates to a 
difference in the percentages of $40.90 \%$, that is, a change from $50 \%$ to $9.09 \%$. A 10 -fold change in the odds from 0.01 to 0.001 is equivalent to a change in the log odds of again 2.30, this time from -4.60 to -6.90 . This however equates to a difference in the percentage of sessions with a quit on a pop-up of $0.89 \%$, from $0.99 \%$ to $0.099 \%$. This is useful to keep in mind when interpreting the following results. Also, as the log odds are generally quite strongly negative it is useful to remember that differences between the small $\log$ odds correspond to limited differences in the probability of quitting on a popup. As most of the log odds are negative it is good to note that this indicates the odds of someone quitting is less than 1 , and the probability is less than $50 \%$.

\section{Results and Discussion}

We fitted the model including all two-way interactions in Rstudio. The backward selection process was completed using Akaike information criterions (AIC) to measure the relative fit of models after the removal of each term. The final model with the lowest AIC included the terms listed in Table 4.1 (an interaction is indicated with ':' between the variables interacting).

Table 4.1 shows the type III Likelihood Ratio test of the final logistic regression model terms. As expected given this combination of terms resulted in the lowest AIC value, each term remaining in this model was a significant predictor of quitting on a pop-up. There were seven main effects and nine interaction terms that significantly influenced whether players quit on a pop-up, adding complexity to the interpretation of results. 
Table 4.1.

ANOVA results of final model type III sum of squares

\begin{tabular}{|c|c|c|c|}
\hline Terms & LR Chisq & Df & P Value \\
\hline EGM Manufacturer & 41.265 & 4 & $<.0001 * * *$ \\
\hline Region & 8.13 & 2 & $0.017 *$ \\
\hline Venue Type & 49.479 & 11 & $<.0001 * * *$ \\
\hline Time of the day & 35.019 & 5 & $<.0001 * * *$ \\
\hline Number of pop-ups in session & 31.17 & 1 & $<.0001 * * *$ \\
\hline Net winnings & 21.217 & 1 & $<.0001 * * *$ \\
\hline Rate of play & 31.799 & 1 & $<.0001 * * *$ \\
\hline EGM Manufacturer:Number of pop-ups in session & 14.321 & 4 & $0.006 * *$ \\
\hline EGM Manufacturer:Net winnings & 26.216 & 4 & $<.0001 * * *$ \\
\hline EGM Manufacturer:Rate of play & 25.592 & 4 & $<.0001 * * *$ \\
\hline Region:Time of the day & 22.064 & 10 & $0.015 *$ \\
\hline Time of the day:number of pop-ups in session & 12.26 & 5 & $0.031 *$ \\
\hline Time of the day:Net winnings & 17.818 & 5 & $0.003 * *$ \\
\hline Number of pop-ups in session:Net winnings & 7.598 & 1 & $0.006 * *$ \\
\hline Number of pop-ups in session:Rate of play & 18.088 & 1 & $<.0001 * * *$ \\
\hline Net winnings:Rate of play & 6.515 & 1 & $0.011 *$ \\
\hline
\end{tabular}


Note. $*$ indicated significance at the 0.05 level, $* *$ at the 0.01 level and $* * *$ indicate significance less than 0.001

Venue type did not interact with any other variables, producing a simple main effect on the log odds of quitting on a pop-up. Figure 4.1 shows this main effect graphically. For this graph and all graphs that follow, the log odds estimates are calculated while averaging over all other variable levels. This graph demonstrates there were significant differences in the likelihood that someone would quit on a pop-up dependent on what type of venue the EGM was in, while holding all other variables in the analysis constant. It displays the significant differences in the log odds of quitting on a pop-up between restaurant and: hotel; and non-commercial workingmen's club. There were also significant differences between non-commercial RSA club and: hotel; restaurant; TAB; and tavern. It should be noted that while these differences were statistically significant, the greatest difference in the log odds was between noncommercial RSA clubs and restaurants with a value of 1.02 . This could be more easily thought of as; the odds of someone quitting on a pop-up while playing an EGM in a restaurant was 2.78 times the odds of someone quitting on a pop-up on an EGM at a non-commercial RSA club. As noted above, the strongly negative log odds indicate that these differences correspond to small differences in the probability of quitting on a popup. 


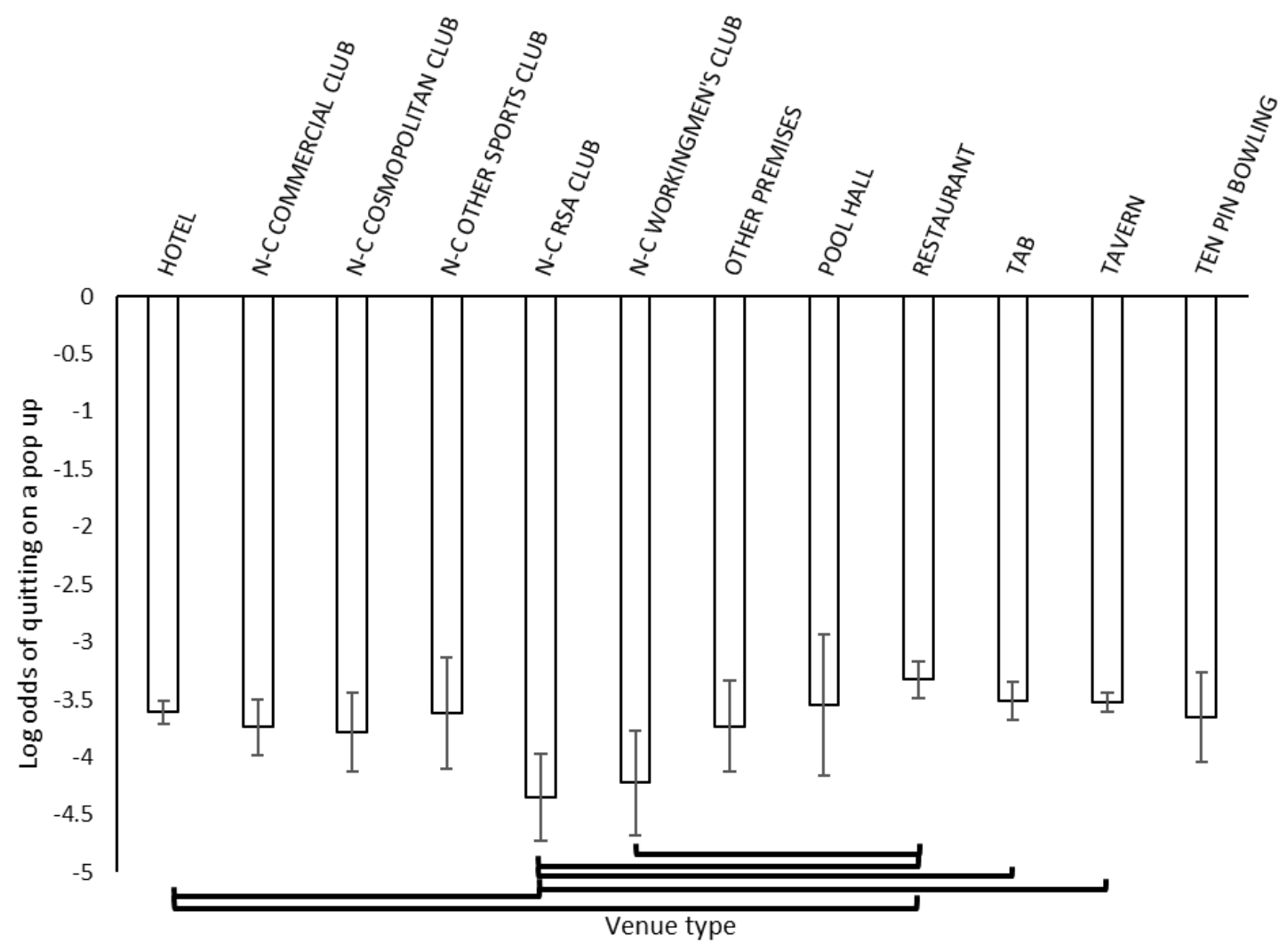

Figure 4.1. Graph displaying main effect of venue type (categorical). Bars show the log odds of quitting on a pop-up across each type of venue. Error bars are 95\% confidence intervals. Statistical indicators (horizontal line) are significant to at least the 0.05 level.

Note. N-C stands for non- commercial.

For ease of interpretation the categorical-categorical interactions are presented below first (log odds of quitting on $y$-axis), followed by categorical-continuous interactions (rate at which log of quitting changes as continuous variable increases on $y$ axis). Note, \# indicates significant difference with all other levels.

The first significant interaction discussed is that between net winnings and manufacturer. This can be visualised in two ways as seen in Figure 4.2; by comparing between manufacturers within sessions with either gain (top) or loss (middle) outcomes, or by comparing sessions with gain or loss outcomes within each manufacturer (bottom). 
The top (gain) and middle (loss) graphs displayed significant differences in the $\log$ odds of quitting on a pop-up between different manufacturers. These differences were not always consistent across sessions with net gain or net loss outcomes. This meant the likelihood of a player quitting on a pop-up on an EGM made by one manufacturer, over an EGM made by a different manufacturer depended on whether the player was winning or losing. An example of this is discussed for clarity. When players were at a net gain, the odds of a player quitting on an AGT EGM were 0.72 times the odds of a player quitting on an Aristocrat machine (at a net gain those on an Aristocrat machine were more likely to quit on a pop-up than those on an AGT machine). However, there was no significant difference between these two manufacturers when the player was at a net loss.

There is an alternative way of looking at this interaction, this is also presented in the bottom graph of Figure 4.2. This graph displays the log odds of quitting on a pop-up between the gain and loss outcomes for each manufacturer separately. This graph shows for all the manufacturers, the log odds of quitting on a pop-up was smaller when the session ended at a loss compared to a net win. Again, the log odds values are quite negative indicating that while there was a significant impact of the interaction between these variables on the likelihood of a person quitting, the absolute probability of quitting did not differ greatly. 

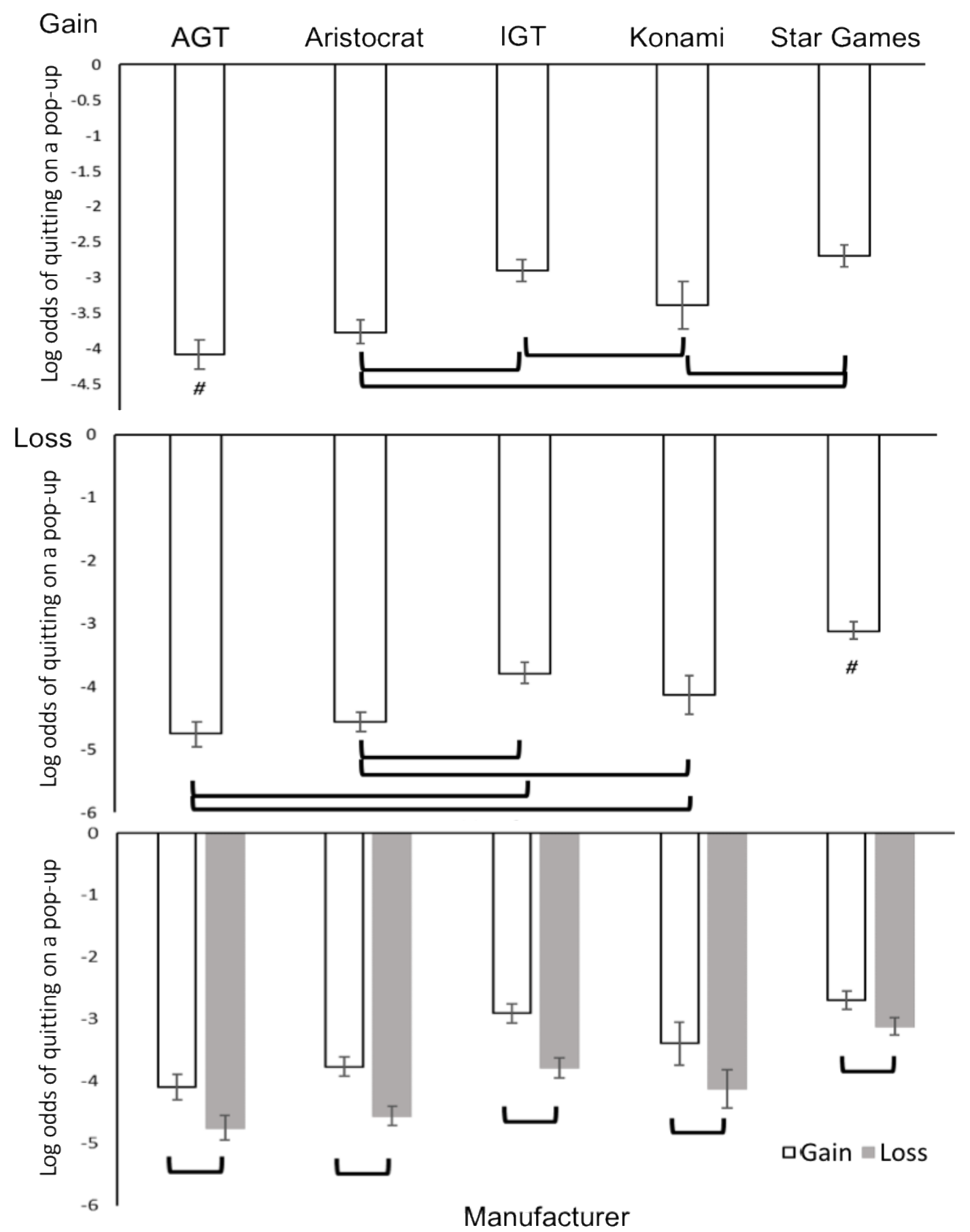

Figure 4.2. Graphs displaying interaction between net winnings (categorical) and manufacturers (categorical). Bars show log odds of quitting on a pop-up. Top two graphs show differences between manufacturers across both gain (top) and loss (middle) outcomes. The bottom graph shows the same data repeated, displaying the other side of the interaction; how log odds differ between gain and loss across each manufacturer. Error bars are $95 \%$ confidence intervals. Statistical indicators are significant to at least the 0.05 level. 
The next significant interaction in the logistic regression model was between region and the time of the day the session took place. This interaction is seen in Figure 4.3. The difference in the log odds of quitting on a pop-up across the times of the day are dependent on what region of New Zealand the session took place (first, second and third graphs). For the Central region there was a significant difference between overnight and all other time of the day categories. This equated to the odds of someone quitting on a pop-up overnight in the Central region being between $1.64-2.16$ times the odds of someone quitting on a pop-up at any other time of the day in the Central region. For the Northern region there were no significant differences between the times of the day. For the Southern region there were two differences, that between evening and both: afternoon; and lunchtime. In the Southern region the odds of quitting on a pop-up in the evening were 1.41 times the odds of quitting in the afternoon, and 1.64 times the odds of quitting at lunchtime.

This interaction is also displayed in reverse; looking at the differences between the three regions, across each time of the day category. This showed a difference in the log odds of quitting on a pop-up between the Central and Northern regions both in the afternoon and after work, and also a difference between Northern and Southern at lunchtime. Again, all the log odds values sit around -4 which are far from zero, therefore differences correspond to limited differences in the probability. 

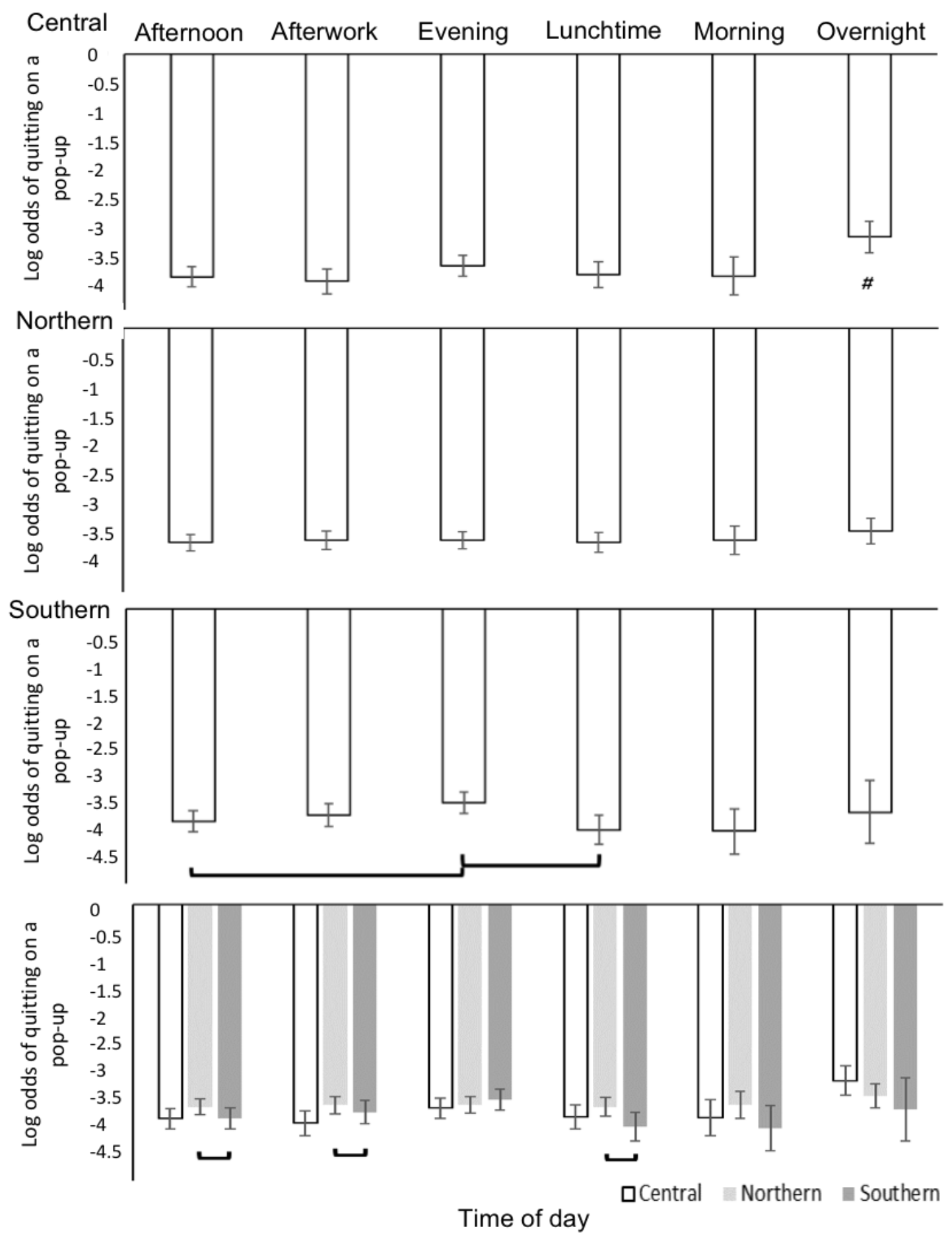

Figure 4.3. Bar graphs displaying interaction between the three regions (categorical) and time of day (categorical). Bars show the log odds of quitting on a pop-up for each time of the day across the Central region (top), Northern region (second) and Southern region (third). The bottom graph shows the same data repeated to show the other side of the interaction; how log odds differ between the regions across each time of the day. Error bars are 95\% confidence intervals. Statistical indicators are significant to at least the 0.05 level. 
The next significant interaction was that between net win/loss outcome and time of day. Figure 4.4 displays this interaction. The first two graphs show that significant differences in log odds of quitting on a pop-up between the times of day, are dependent on whether the session had a net win (top) or a net loss (middle). Of interest, none of the differences found to be significant for the gain outcome, were significant for the loss outcome. The third graph shows the interaction in an alternative view; the log odds of quitting on a pop-up was always greater for sessions ending with a net gain than for sessions ending with a net loss. For clarity, the odds of quitting on a pop-up with a gain outcome at all times of the day were between 1.44-2.80 times the odds of quitting on a pop-up on a loss outcome. Again, all the log odds values sat around -4, so these differences corresponded to limited absolute differences in the probability of quitting. 


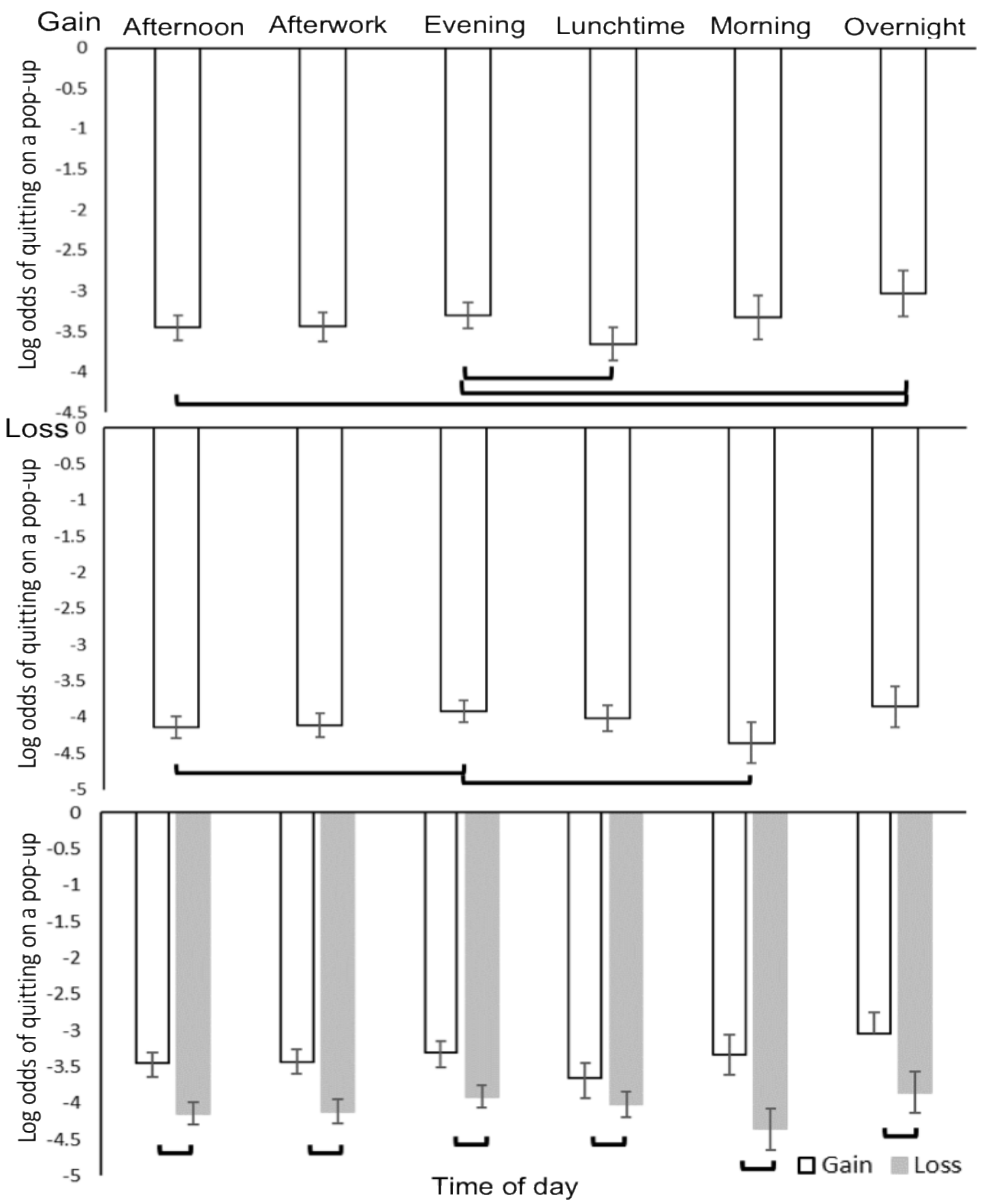

Figure 4.4. Bar graphs displaying interaction between the net winning outcome (categorical) and time of day (categorical). Bars show log odds of quitting on a pop-up for each time of day across the gain (top) and loss (middle) session outcomes. This interaction can be displayed in an alternative view showing the differences between gain and loss at each time of the day (bottom). Error bars are 95\% confidence intervals. Statistical indicators are significant to at least the 0.05 level. 
The following interactions are those between categorical and continuous variables. Note the change in the $y$-axis when interpreting graphs.

Another significant interaction was that between the number of pop-ups seen in a session and the manufacturer. This is displayed in Figure 4.5. As the number of popups seen in a session increased, the log odds of quitting on a pop-up decreased more rapidly for IGT than they did for Aristocrat. It should also be noted that despite differences, each additional pop-up reduced the log odds of quitting on a pop-up across all manufacturers.

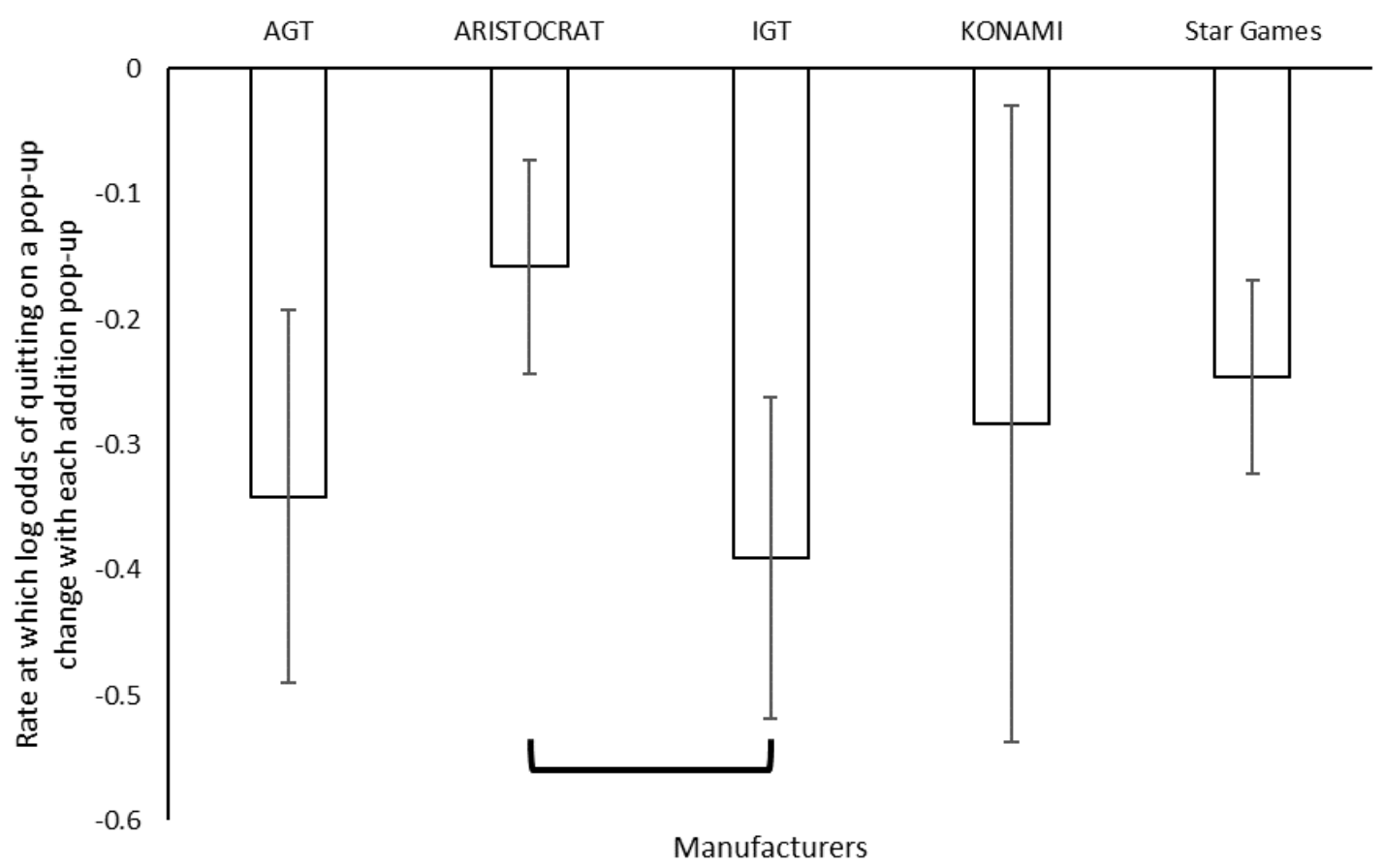

Figure 4.5. Bar graph displaying interaction between number of pop-ups (continuous) and manufacturers (categorical). Bars show the rate at which the log odds of quitting on a pop-up changed as the number of pop-ups appearing in the session increased, across each manufacturer. Error bars are 95\% confidence intervals. Statistical indicators are significant to at least the 0.05 level. 
The next significant interaction was that between net winnings and number of pop-ups seen in the session. Figure 4.6 shows this interaction. There was a significant difference in the rate at which the log odds of quitting on a pop-up changed between sessions that ended with a net gain and sessions that ended with a net loss. Each additional pop-up in the gain outcomes reduced log odds of quitting on a pop-up more rapidly than in the loss outcomes. In other words, for sessions that ended with a net gain, the likelihood of quitting on a pop-up decreased faster with each additional popup, when compared to sessions that ended in a net loss.

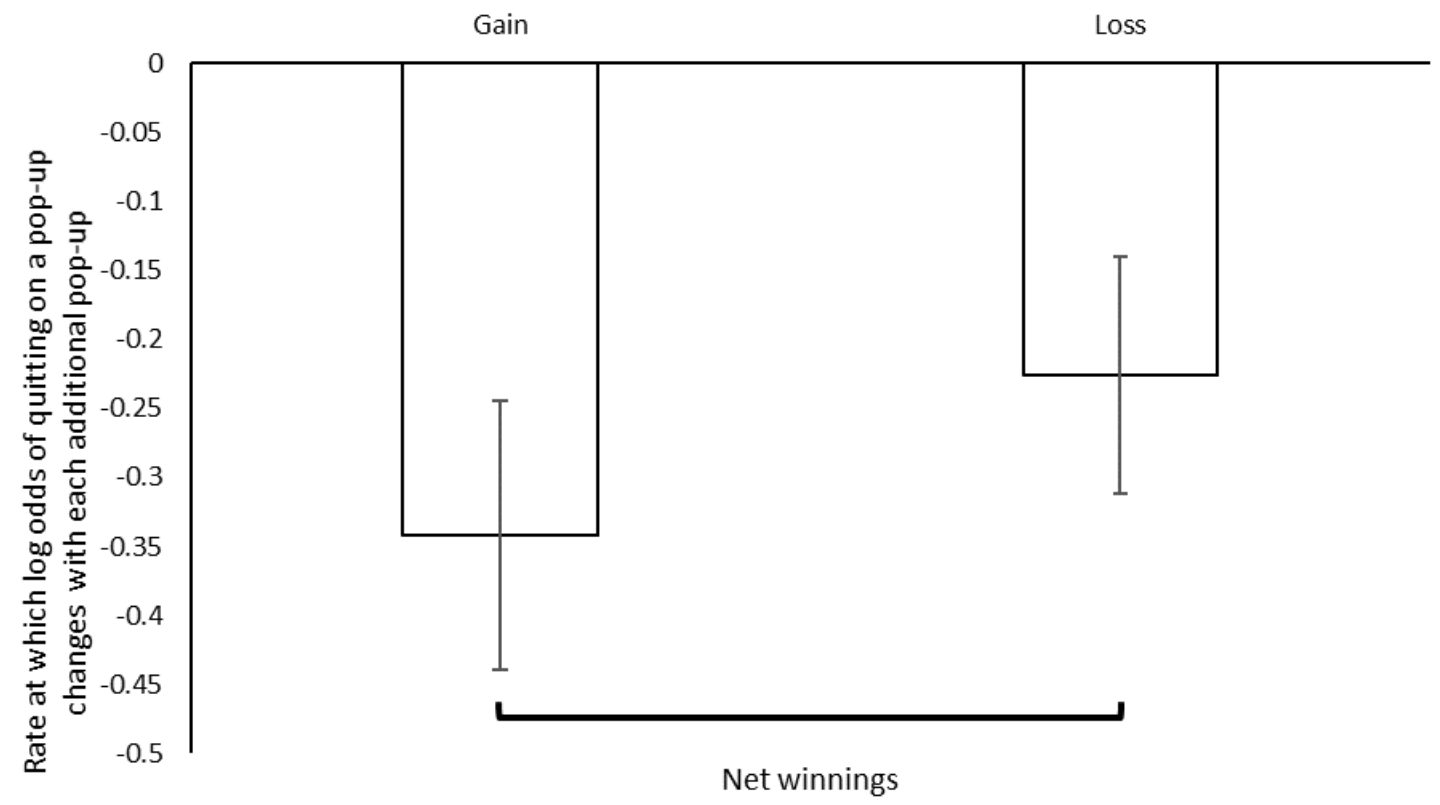

Figure 4.6. Bar graph displaying interaction between number of pop-ups in a session (continuous) and net winnings (categorical). Bars show the rate at which log odds of quitting on a pop-up changed as the number of pop-ups seen increased, across gain and loss outcomes. Error bars are 95\% confidence intervals. Statistical indicators are significant to at least the 0.05 level.

The next interaction was that between time of the day and number of pop-ups seen. Despite the fact that this interaction was significant, there were no significant 
differences in the rate at which log odds of quitting on a pop-up changed as the number of pop-ups seen increased, across the different times of the day. This can be seen in Figure 4.7. The correction for multiple comparisons was the most likely reason for the overall interaction being significant, while individual pairwise tests did not reach the significance threshold. As seen for all manufacturers and both net gain categories, each additional pop-up reduced the chance of quitting on a pop-up across all times of the day.

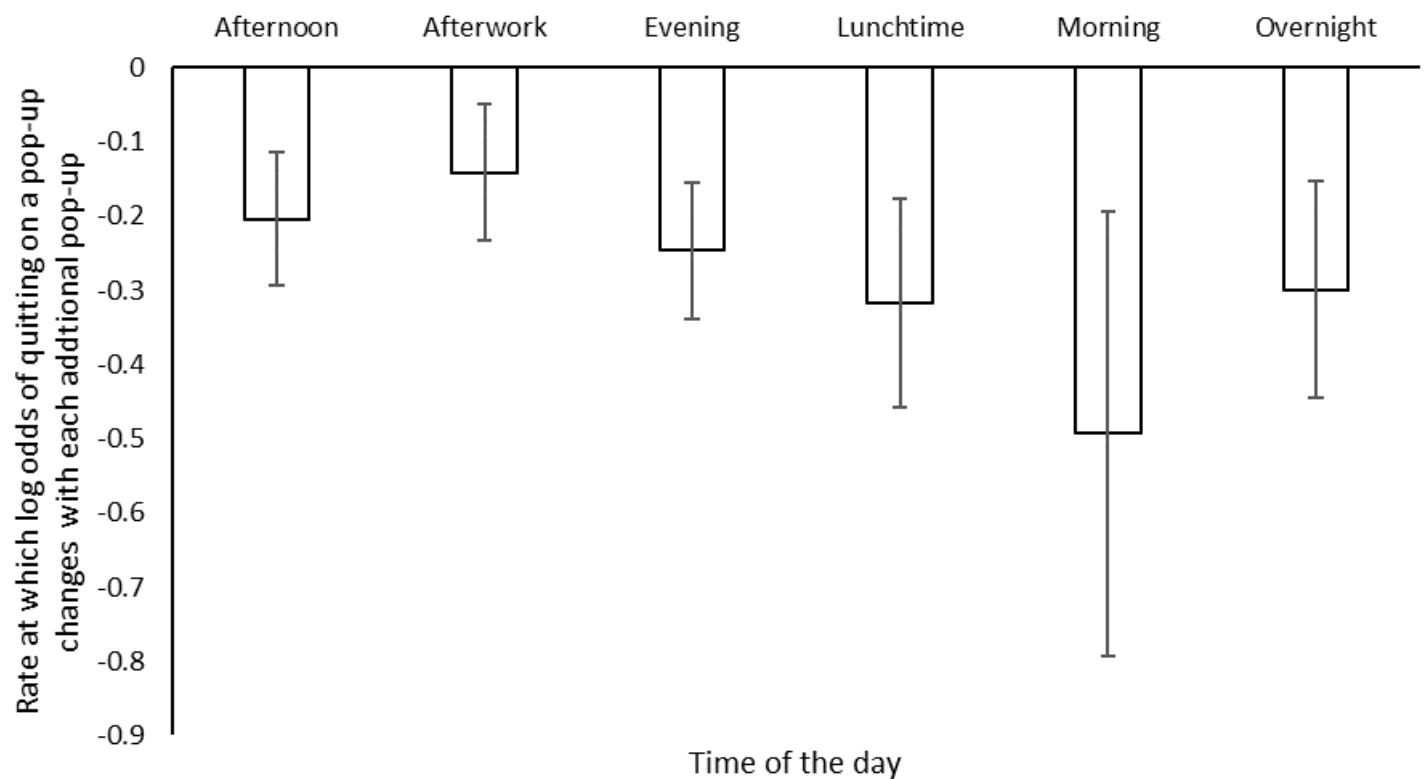

Figure 4.7. Bar graph displaying interaction between number of pop-ups seen (continuous) and time of day (categorical). Bars show the rate at which log odds of quitting on a pop-up changed as the number of pop-ups seen increased, across the times of the day. Error bars are $95 \%$ confidence intervals.

The next significant interaction was between net outcome and rate of play (dollars per minute). This is seen in Figure 4.8. There was a significant difference in how the log odds of quitting on a pop-up changed as the rate of play increased between the gain and loss outcomes. The loss sessions resulted in a more rapid decrease in log odds of quitting on a pop-up as rate of play increased, compared to the gain outcomes. 


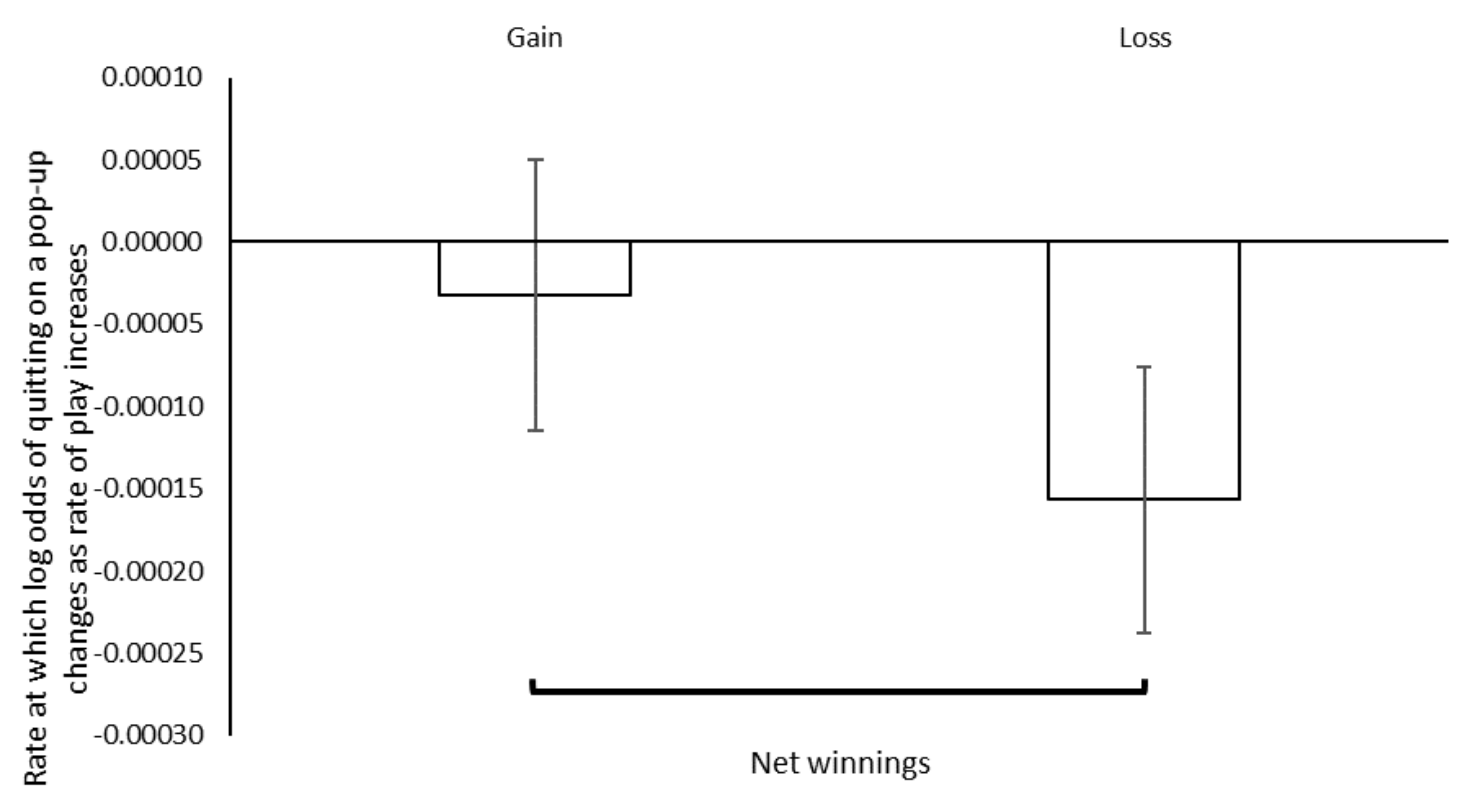

Figure 4.8. Bar graph displaying interaction between rate of play (continuous) and net winnings (categorical). Bars show the rate at which log odds of quitting on a pop-up changed as the rate of play increased, across gain and loss outcomes. Error bars are $95 \%$ confidence intervals. Statistical indicators are significant to at least the 0.05 level.

The next significant interaction was that between the rate of play and manufacturer and can be seen in Figure 4.9. For all manufacturers there was very little change in the log odds of quitting as the rate of play changed (note the scale on the $y$ axis). In this context, as the rate of play increased there was an increase in log odds of quitting on a pop-up for players on Konami machines, while this led to a decrease in the $\log$ odds for players on all other machines. The other difference was between AGT and Star Games where log odds of quitting on a pop-up, decreased more rapidly for AGT machines compared to Star Games machines, as the rate of play increased. There was no obvious explanation as to why Konami displayed an opposite trend to the other manufacturers. It is possible this difference was only significant due to the large sample size but did not reflect a meaningful difference as the difference is minimal. 




Figure 4.9. Bar graph displaying interaction between rate of play (continuous) and manufacturers (categorical). Bars show rate at which log odds of quitting on a pop-up changed as the rate of play increased, across each manufacturer. Error bars are 95\% confidence intervals. Statistical indicators are significant to at least the 0.05 level.

The final significant interaction was that between the number of pop-ups seen and the rate of play. As both variables were continuous variables, bar graphs could not be produced. The coefficient was positive for this interaction indicating the number of pop-ups seen had a less negative effect on the log odds of quitting on a pop-up, the higher the rate of play. This can also be described as, the rate of play had a less negative effect on the log odds of quitting on a pop-up, the more pop-ups that were seen.

Following the specific findings of the model coefficients, we tested how accurately this model predicted when a player quit on a pop-up. We first conducted this on the training dataset ( $75 \%$ of the data) used to create the model, and second on the test dataset (the remaining 25\%) which was new to the model. This tested the validity of the model. A confusion matrix was used to test prediction accuracy and indicated how well 
the model could predict session outcomes; quit on a pop-up or at any other time. The confusion matrix for the training dataset is displayed in Table 4.2 and for the test dataset in Table 4.3. This matrix was set with a threshold of 0.5 for predicting outcomes and showed for both datasets (the $75 \%$ and $25 \%$ ), no combination of predictor values was strong enough to predict a quit on a pop-up response. Changing the threshold from 0.5 did not change the results. The model's lack of ability to predict quitting on a pop-up was not surprising given the dataset the model was created from. For both datasets predicting no quit on pop-up responses produced an accuracy of between $96.8 \%$ (test dataset) $-96.9 \%$ (training dataset). This was because there were very few times when a player quit on a pop-up.

Table 4.2.

Confusion Matrix for training dataset

Observed

\begin{tabular}{lrrr} 
& & & \\
\cline { 2 - 4 } Predicted & No & Yes & \\
\hline No & $89960(96.9 \%)$ & $2884(3.1 \%)$ & 92842 \\
Yes & 0 & 0 & 0 \\
& & & \\
\hline & 89960 & 2884 & \\
\hline
\end{tabular}


Table 4.3.

Confusion Matrix for test dataset

\begin{tabular}{lccr}
\hline & \multicolumn{3}{c}{ Observed } \\
Predicted & No & Yes & \\
\cline { 2 - 4 } & & & \\
\hline No & $29957(96.8 \%)$ & $991(3.2 \%)$ & 30948 \\
Yes & 0 & 0 & 0 \\
& & & \\
\hline & 299 & 991 & \\
\hline
\end{tabular}

This situation where all responses are predicted as non-events by a logistic regression model is a known issue using data with rare events. This is because the most accurate way to classify responses is to make all predictions a non-event given that this makes the vast majority of predictions correct. This problem is common in fields such as international relations, specifically global conflict prediction. Things such as wars are rare events but the cost of inaccurately predicting them could be very high (King \& Zeng, 2001a). Researchers have attempted to reduce the influence that the non-events have on the final model in logistic regression analysis, in order to get more accurate predictions of events in rare event datasets (King \& Zeng, 2001b). The most popular solution to this was an adjustment produced by King and Zeng (2001b) called 'logistic regression with rare event data'. This analysis adjusts for the bias that abundance of non-events, and lack of rare events produce.

We investigated this as a possible solution to the current inability to predict any events. King and Zeng (2001b) however, specify that their analysis adjustment is effective for low frequency events, but as the absolute number of events increases 
(while the proportion of overall events may still be low), the adjustment has less and less impact. They comment, for a sample size with 20,000 data points and $200(1.1 \%)$ events, the impact the adjustment makes over the regular analysis is $0.2 \%$. For the current study with 123,790 data points and 3,875 events, this adjustment was expected to have limited impact on our results. Despite this we ran the rare event logistic regression model adjustment on our data to check whether it could improve our ability to predict the quitting on a pop-up event. As expected the adjustment provided very little change to the results and, most importantly, there was no improvement in the ability of the model to predict an event (number of events predicted was still 0 ). This provided support for the theory that our lack of predictive ability for a single event was not just an artefact of the bias a high proportion of non-events produces, but actually a result in itself. That is, no combination of variables was strong enough to predict a player quitting on a pop-up.

With so many interaction terms there was a risk of overfitting of the model. This risk was reduced given the sample size, however this possibility was still explored. We fitted an alternative model removing all interaction terms, but leaving variables as main effects and tested its predictive ability with a confusion matrix. This model continued to result in 0 event predictions, indicating that overfitting was not causing the model's lack of ability to predict quitting on a pop-up.

For the purposes of the questions we wanted to answer, we assumed independence of all sessions however this could not be verified given the dataset. We had no information on which individuals played each session, making it likely that a number of players contributed multiple sessions to the dataset. It was also known that repeated sessions contributed by the same machines were present in the dataset. Given that there were 8,840 machines, computing limitations meant we could not run an 
analysis that allowed for clustering across these. For simplicity we assumed independence of all sessions but acknowledge this could have influenced results and could be something future research could investigate. It is possible positioning of machines within a venue could have consistently impacted play and therefore all sessions on one machine could have been correlated. 


\section{Chapter 5: Elective Displays, Study 3}

While there is little specific research on elective information displays in the literature, we can gain useful insight from a combination of other related literature. The research on pop-up display content summarised in the introduction above can provide useful information about the type of information presented on elective displays. Using this, and research on differences between solicited and unsolicited information, comments can be made on how effective elective player information displays work in New Zealand. As discussed in the introduction, New Zealand elective information displays include the same information as pop-up displays along with additional information. This is the odds of winning each game, including the top and bottom 5 winning combinations (value of prize), the RTP rate as a percentage and the maximum and minimum spend per spin (using the maximum/minimum number of lines and maximum/minimum bet per line). This additional content means that, not only do elective displays contain feedback information about the session, they also contain accurate information about the likelihood of winning which could reduce false cognitions. Elective displays might therefore be more effective at increasing responsible gambling because gamblers receive both types of information.

There is a key important difference however, between elective displays and popup displays. While pop-ups appear automatically as a result of gambling play, elective displays are only seen when they are accessed by choice. Research conducted outside the gambling setting showed people react to information sought out themselves, in different ways to information that is presented without their request. Fitzsimons and Lehmann (2004) looked at the effect unsolicited advice had on behavioural outcomes. They found unsolicited advice that contradicted what the participant already thought encouraged behaviours contrary to recommendations. Research by Van Swol, 
MacGeorge, and Prahl (2017) also suggested people had higher intentions of using advice they had given permission to receive, compared to advice provided without their asking. This finding was specific to advice on issues participants rated as personal, which could be the case with gambling. This would again suggest elective information displays should be effective, perhaps more so than pop-ups, because the person seeks out the information, and is therefore more likely to use it.

Wood and Wohl (2015) observed this occurring in the gambling context. They examined the impact of feedback on play which players had opted to receive. They found, for at risk players who had chosen to receive feedback, providing session information led to significantly reduced expenditure compared to those who had not engaged with the tool. This suggested when players choose to receive feedback, it can be effective at reducing gambling spending.

It must however be considered that even if the content of these elective displays was effective, for this effect to occur, the player must request to see the elective display. This means assessing whether elective displays would be effective, would first require assessing whether gamblers access them at all.

Palmer du Preez et al. (2014) investigated elective information displays in New Zealand. In a survey they found 69\% of EGM gamblers were aware of electives, with $38.7 \%$ indicating they used them rarely/sometimes, and $7.6 \%$ of people used them often/always. While their survey indicated a third of gamblers thought elective displays help them control the amount of time and money spent gambling, their focus group research also showed that some players use the displays as part of their potentially harmful gambling strategies. These strategies included looking for machines with the highest RTP. This did not appear to be a harmful technique, however they noted this 
could encourage beliefs that EGMs could be played in strategic ways, leading to the perpetuation of false illusions surrounding the control of EGM outcomes. These displays could feed further into player's false perceptions as they emphasise which machines have increased rates of winning, while the advantage is, in reality, minimal. There is a suggestion that player's see the machines with highest RTP as an indication they are likely to win, however in practice they are still more likely than not to lose the money invested; all RTPs are below 100\%. This links these displays with unintended consequences related to cognitive distortions and problem gambling. This is useful to be aware of going forward and investigating the extent to which elective displays minimise harm in New Zealand.

The current study addressed a number of key questions to evaluate the potential utility of elective displays as harm minimisation tools. The first was: how often do players access them? If we find players use them very infrequently, the quality of the information displayed on them is irrelevant. For this reason the current study aims to build on Palmer du Preez et al.'s (2014) study. We first investigated the rate at which these displays were used, and second, determined how rates of access varied by manufacturer and day of the week, and third, determined what additional analyses would be possible using the data associated with elective displays accessed.

\section{Method}

The same dataset described in the preface to Study 1 was used to investigate the questions about the elective information displays. The key recorded measure for this study was the 'EGM PID accessed' daily count (note that PID stands for Player Information Display, but in the context of 'PID accessed' this only refers to the elective displays). This variable is a count of the total number of times an elective information 
display had been viewed on each individual EGM, each day measured from 2am- 2am (this measure of a day is a convention within the EMS). This information was collected within the same week-long period as the "EGM PID session stopped" event data described above. The 'EGM PID accessed' measure differed however to the event related data ('EGM PID session stopped'). Instead of recording access to elective displays as they occurred with a time stamp, EMS only recorded the tally for each machine, each day. This meant information about the time at which each elective display was accessed was not available and therefore it was not possible to link individual accesses to elective displays with specific gambling sessions as we could when pop-ups resulted in quitting. Information such as the rate of play and the net spend was therefore not available, but other information not directly linked to the sessions was, such as the day of the week and manufacturer.

\section{Results and Discussion}

\section{Elective Information Displays Access Frequency}

Throughout the week the elective displays were accessed a total of 7,275 times. Over all machines, elective displays were accessed less than once a day (mean $=0.17$ displays, median $=0, S D=1.72$, minimum $=0$, maximum $=49)$. For the sake of comparison to pop-up displays this value can be presented as a percentage of the number of sessions in the week. If we assume each elective display was accessed in a new session giving the most inflated percentage possible, the percentage of sessions on which a player accessed an elective display would be $0.66 \%$. As it is possible players access this display multiple times in a session this percentage could only get smaller with more refined information. 
Another way to present the occurrence of players accessing elective displays was as a rate. That is, the mean number of displays accessed per hour of total play time. The total number of hours played across the week was 182,730 hours which is $12.30 \%$ of the total number of hours of potential play per week. With 7,275 displays accessed during the week the access rate was 0.0398 displays per hour of total time spent playing. Overall, elective displays were accessed only rarely, and thus are unlikely to be achieving the goal of reducing gambling harm.

\section{Distribution of Access to Elective Displays}

The second goal relating to elective information displays was to investigate the distribution of elective information display accesses broken down by both day of the week and manufacturer. Table 5.1 shows that, while the number of elective displays accessed fluctuated across the week, the rate of access per hour of play remained stable across the week ranging from $0.042-0.049$. As the data had a non-normal distribution, a Kruskal-Wallis test was conducted to evaluate differences among the days of the week in the rate of electives accessed. The test produced a significant result $H(6)=191.27, p$ $<.001$. Follow-up tests were conducted to evaluate pairwise differences among the seven days of the week, controlling for Type I error across tests by using the $\mathrm{BH}$ approach. The results of these tests are shown in Table 5.2, indicating many significant differences between the days of the week, predominantly Sunday and Monday differing from other days of the week. 
Table 5.1.

Elective information displays accessed by days of the week

\begin{tabular}{|c|c|c|c|c|c|c|c|}
\hline & Mon & Tues & Wed & Thurs & Fri & Sat & Sun \\
\hline Total sessions & 109,363 & 147,220 & 176,078 & 184,294 & 191,733 & 177,041 & 117,745 \\
\hline \multicolumn{8}{|l|}{ Total displays } \\
\hline accessed (\% & 692 & 997 & 1218 & 1276 & 1301 & 1067 & 723 \\
\hline of sessions) & $(0.63 \%)$ & $(0.68 \%)$ & $(0.69 \%)$ & $(0.69 \%)$ & $(0.68 \%)$ & $(0.60 \%)$ & $(0.61 \%)$ \\
\hline \multicolumn{8}{|l|}{ Total playing } \\
\hline minutes & $1,048,617$ & $1,405,286$ & $1,774,886$ & $1,885,982$ & $1,970,025$ & $1,751,729$ & $1,127,279$ \\
\hline (hours) & $(17476.9)$ & $(23421.4)$ & (29581.4) & $(31433.0)$ & $(32833.8)$ & (29195.5) & (18788.0) \\
\hline
\end{tabular}

Mean displays

per hour

(within

$\begin{array}{lllllll}0.04155 & 0.04820 & 0.04565 & 0.04315 & 0.04668 & 0.04204 & 0.04864\end{array}$

sessions)

Note. Mean displays per hour are calculated from averaging displays per hour rates within sessions, rather than using weekly total displays and total hour values.

These significant differences in the rates that electives were accessed could have indicated different types of gambling occurring on each day. The highest rates of access occur on Tuesday and Sunday. Identifying the characteristics of players that tend to play on these days (problem gamblers versus recreational gamblers), would indicate the type of players most likely to access elective displays. It was also possible that due to the large sample size these differences were significant but not meaningful. Observing the 
absolute size of rates across the days of the week, the absolute numbers are quite similar, much more similar than differences seen across the manufacturers below. This suggested that while there may have been a significant effect of weekday on the rate at which these were accessed, these differences were minor and other variables would be better to explore further.

\section{Table 5.2}

Matrix of significance values for pairwise comparisons across the day of the week

\begin{tabular}{lllllll}
\hline & Tuesday & Wednesday & Thursday & Friday & Saturday & Sunday \\
Monday & $<0.001^{* * *}$ & $<0.001 * * *$ & $<0.001^{* * *}$ & $<0.001^{* * *}$ & $<0.001 * * *$ & 0.206 \\
Tuesday & - & $<0.001 * * *$ & $<0.001 * * *$ & $<0.001 * * *$ & $0.039^{*}$ & $<0.001^{* * *}$ \\
Wednesday - & - & 0.975 & 0.485 & 0.083 & $<0.001^{* * *}$ \\
Thursday & - & - & - & 0.485 & 0.083 & $<0.001^{* * *}$ \\
Friday & - & - & - & - & $0.014^{*}$ & $<0.001^{* * *}$ \\
Saturday & - & - & - & - & - & $<0.001^{* * *}$ \\
\hline
\end{tabular}

Table 5.3 shows the same information as Table 5.1 however separated by manufacturer. Similar to days of the week, there was large variability in the absolute number of times an elective was accessed across manufacturers. However, unlike the days of the week, this large variability remained when observing the rates of electives accessed per hour across manufacturers. The differences in access rate between manufacturers ranged from $0.03121-0.06026$ displays per hour. A Kruskal-Wallis test was conducted to evaluate differences in access rate between manufacturers. The test produced a significant result $H(4)=221.07, p<.001$. Follow-up tests were conducted to evaluate pairwise differences among the five manufacturers, controlling for Type I error 
across tests by using the BH approach. The results of these tests are shown in Table 5.4, indicating many significant differences between the manufacturers. This finding indicated a potential difference in how often electives were accessed based on the manufacturer that developed them. While it was unclear what specific design choices made by the manufacturers could have affected how frequently gamblers elected to view the displays, the design of the button is one possibility. The information button could be more prominent on some machines encouraging engagement with it. The display itself could also have impacted access, however, as these displays are only seen by choice the design would only influence repeated access by the same gambler.

\section{Table 5.3.}

Elective information displays accessed by manufacturers

\begin{tabular}{llllll}
\hline & AGT & Aristocrat & IGT & Konami & Star Games \\
\cline { 2 - 6 } Total sessions & 179,725 & 383,740 & 217,764 & 98,360 & 224,437
\end{tabular}

Total displays

$\begin{array}{llllll}\text { accessed }(\% & 817 & 2347 & 1225 & 687 & 2198 \\ \text { of sessions }) & (0.45 \%) & (0.61 \%) & (0.56 \%) & (0.70 \%) & (0.98 \%)\end{array}$

Total playing

minutes

$$
1,570,640 \quad 4,179,562
$$

758,118

(hours)

$$
(26,177)
$$

Displays

accessed per

$$
\begin{array}{lllll}
0.03014 & 0.04003 & 0.03533 & 0.08218 & 0.06192
\end{array}
$$

hour (within

sessions) 


\begin{abstract}
Note. Mean displays per hour are calculated from averaging displays per hour rates within sessions, rather than using weekly total displays and total hour values.
\end{abstract}

\title{
Table 5.4
}

Matrix of significance values for pairwise comparisons across manufacturers

\begin{tabular}{lllll}
\hline & Aristocrat & IGT & Konami & Star Games \\
AGT & $<0.001 * * *$ & 0.48398 & $0.00466^{* *}$ & $<0.001 * * *$ \\
Aristocrat & - & $<0.001 * * *$ & $<0.001 * * *$ & $<0.001 * * *$ \\
IGT & - & - & $0.01096^{*}$ & $<0.001 * * *$ \\
Konami & - & - & - & $<0.001 * * *$ \\
\hline
\end{tabular}

These rates however, should not be looked at in isolation. The elective displays contain some identical information to that found on pop-up displays, therefore making it essential to also investigate a potential relationship between elective display usage and the times that pop-ups appear. Having seen a pop-up display that contains much of the same information as the elective display, could reduce a player's likelihood of electing to view the display. Figure 5.1 presents the rate of elective displays accessed per hour with pop-up onset times overlaid over each manufacturer's bar. If the pop-up displays were impacting the access rate of electives, we would expect a systematic relationship between manufacturer and onset time. That is, manufacturers with an earliest onset time (more pop-ups seen) would have the smallest rate of electives accessed per hour, while those with the longest onset time (less pop-ups seen) would have the greatest use of electives. From Figure 5.1 there is no clear trend reflecting this explanation. Given that Aristocrat and Star Games which have the same onset times, have two distinct rates of accessing elective displays, it further demonstrates this explanation cannot fully account for these differences. The next step would be to investigate how other variables 
influenced access to electives and assess whether differences found between manufacturers held once controlling for other variables.

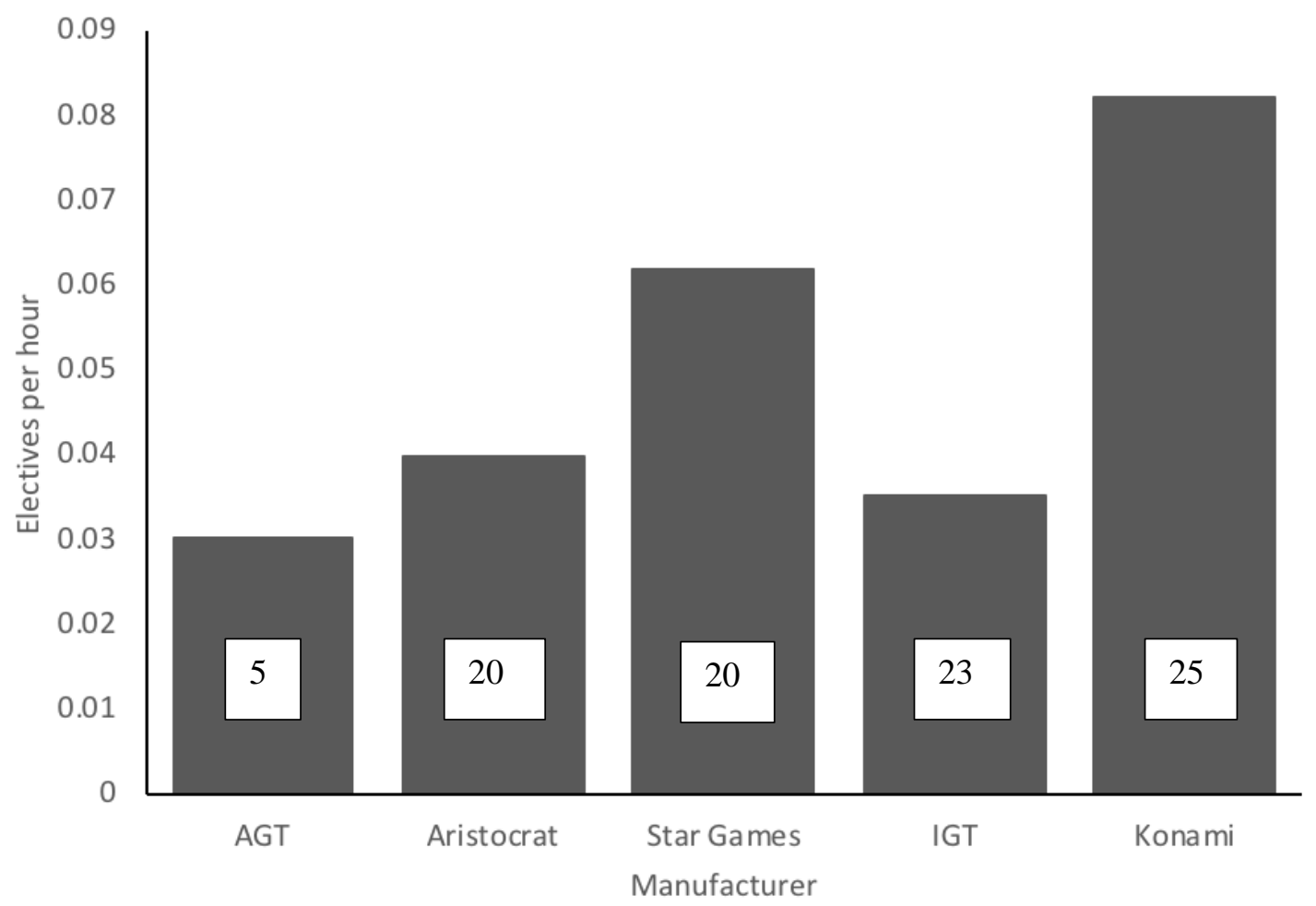

Figure 5.1. Graph showing elective display access rate across the manufacturers with pop-up onset times overlaid. 


\section{Chapter 6: Study 4}

Study 4 aimed to identify which variables influenced access to elective displays. Like Study 2, we had very little prior knowledge about what would significantly predict this behaviour. Study 4 indicated, while day of the week only had a small effect on rate of accessing electives, manufacturer appeared to have a large effect. The current study tested whether these findings persisted when controlling for other available variables.

As this study was exploratory, we included as many variables as possible in the analysis to see whether we could predict individual EGMs on which elective displays were more likely to be used. The "PID accessed" meter information was only a tally for the day, meaning that it was not possible to identify the session or sessions during which the display was accessed. Therefore, information about the specific sessions could not be linked to the PID access information. The only information available that could be linked was: EGM model (the name of the game/set of games available on the machine), manufacturer of the machine, day of the week, and the region and venue type the EGM was in. We controlled for the number of hours the EGM was in use during the week to be confident that differences in access to elective displays was not a product of the overall busyness of the EGM.

\section{Method}

To control for machine busyness, we created a variable for the total number of hours played on each machine for the week from the session information described above in studies on pop-up displays.

Due to the low frequency of access to elective displays across the week, one day was not long enough to precisely estimate the rate at which electives were accessed. To make this estimate more precise, we calculated the number of electives accessed across 
the seven days of the week, one for each of the 8,840 EGMs. An example of why this was done is given for clarity. An elective display on one particular EGM could be accessed three times on Monday, once on Friday, and zero times on each other day. We considered access to elective displays on this machine was not captured well by any single day, but rather by it being accessed four times across the whole week. One potential limitation of this approach was that we found some significant differences in access rate across the days of the week (see Study 3). However, these were very small in size (see Table 5.1) and the statistical significance of these differences likely reflected the large sample size rather than meaningful differences across days.

The distribution of the number of times the elective displays were accessed across the week was non- normal with a large number of zeroes (i.e. large number of machines on which no gambler accessed the elective display during the week, see Figure 6.1). After exploring a range of analyses that could have been suitable for modelling an outcome variable with this distribution, we determined that the most appropriate approach would be to split the analysis in to two parts: a logistic regression to model the distribution of zeroes and a multiple regression to model access rate for machines with one or more accesses.

Logistic Regression. We first used a logistic regression to determine when people were likely to access the electives versus not access them at all. The binary outcomes were coded as 'accessed' (total access of one or greater) and 'did not access' (total access of zero).

Multiple Regression. Following that analysis, we used a multiple regression to determine what influenced the number of times electives were accessed per hour, given 
that they had been accessed at least once. For this analysis we log transformed the number of elective accesses (due to non-normal distribution of residuals).

\section{Selection and Categorisation of Predictor Variables}

The variables of interest for the logistic and multiple regression were identified and isolated. This was all the variables we had access to that could be linked up with the access totals. Like the logistic regression in Study 2 we needed to ensure there were enough observations in each combination of variable levels. To achieve this it was necessary to collapse across some venue types. The initial dataset categorised each venue as one of 14 types (the same 14 types as in Study 2), we reduced these to five venue types. The hotel, restaurant and tavern categories had enough sessions without collapsing so were left as they were. We created a category for "other premises" which was made up of: other commercial premises; other non-commercial premises; pool halls; TABs; and ten pin bowling premises. We also created a category for "clubs" which was made up of: non-commercial bowling clubs; non-commercial commercial clubs; non-commercial cosmopolitan clubs; non-commercial other sports clubs; noncommercial RSA clubs; and non-commercial workingmen's clubs.

Like the logistic regression conducted for the pop-up displays, we wanted to test how the model performed at predicting actual rates of elective displays accessed with the data. We first tested the model's performance on the training dataset $(75 \%$ of the dataset we used to create the model) and then on the test dataset (the remaining 25\%).

\section{Results and Discussion}

We first assessed the distribution of number of accesses to electives along with the distribution of elective display access rates in order to identify which statistical test 
would be appropriate for the data. These are seen in Figure 6.1 and Figure 6.2

respectively. Note these data are prior to collapsing across days of the week.

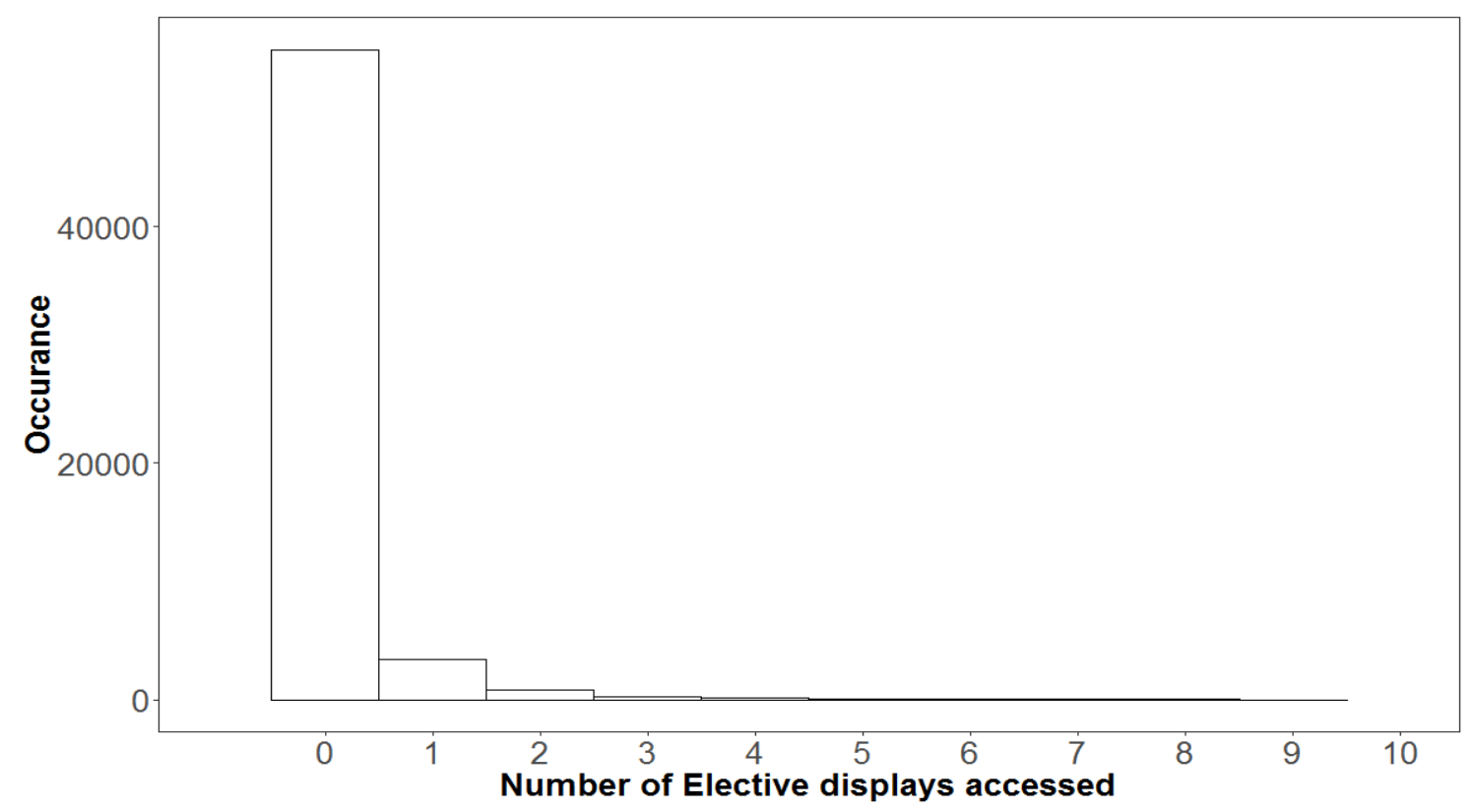

Figure 6.1. Histogram displaying distribution of number of elective displays accessed.

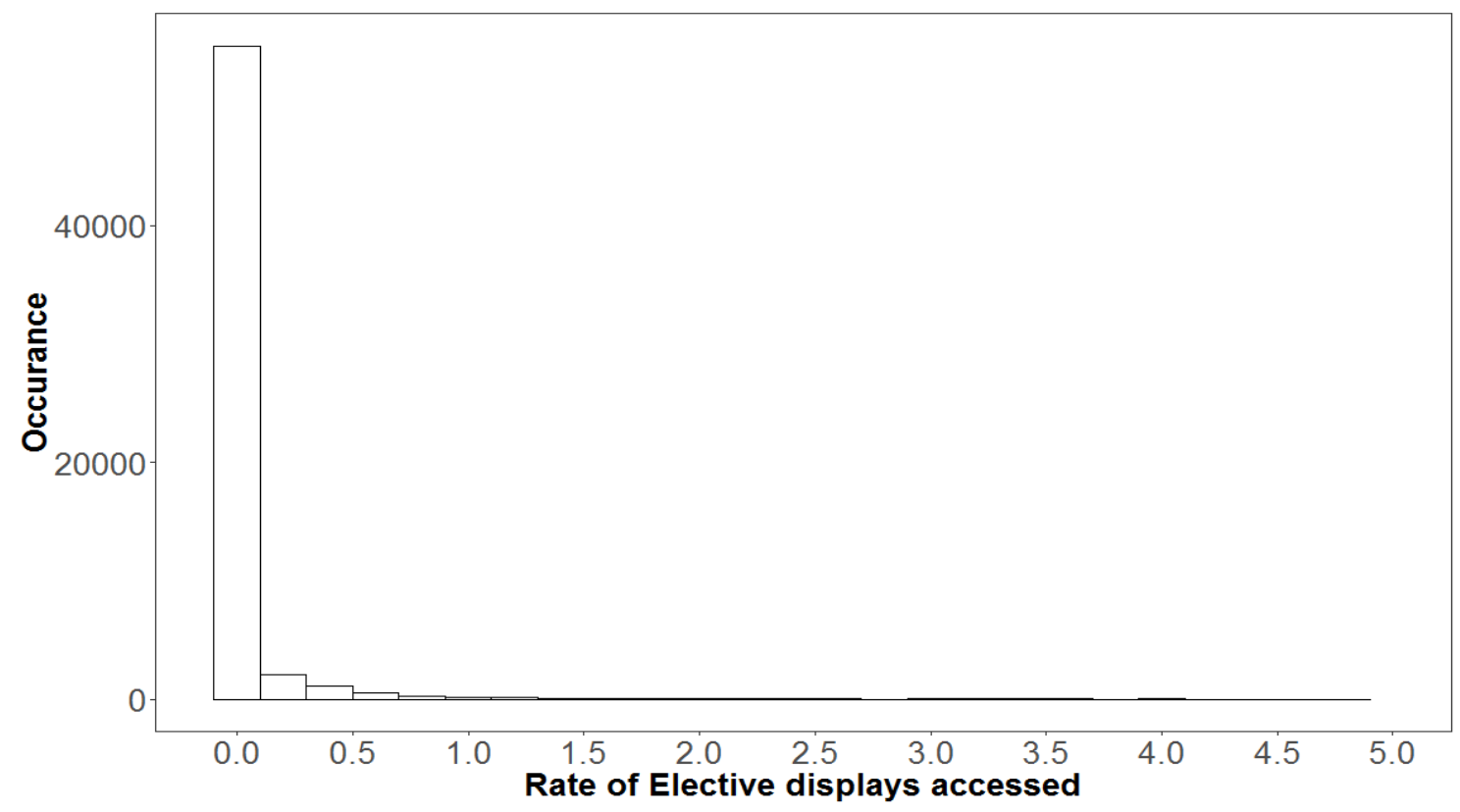

Figure 6.2. Histogram displaying distribution of the rate of elective displays accessed.

\section{Logistic Regression}

Like Study 2 we started with the most complex version of the model and conducted a backwards selection process using AIC values to determine which variables 
contributed significantly to predicting whether or not the displays were accessed. These were: EGM model; manufacturer; region; venue type; and total number of hours played. Because EGM models are produced by specific manufacturers, there was collinearity between these variables and therefore one had to be removed. Because we had already seen differences across manufacturers in Study 3 and had chosen manufacturer over model in Study 2, for comparability we chose to exclude EGM model from the analysis.

The most complex statistical model we started with was a three-way interaction between manufacturer, region, and venue type with total hours played added as a main effect to ensure effects were not an artefact of more time spent playing. This backwards selection process using AIC values resulted in a final model of three main effects: venue type; manufacturer; and total hours played. A likelihood ratio test was used to confirm that each term was statistically significant, see Table 6.1. All terms retained in the equation during the backwards selection process were significant predictors of whether a machine recorded at least one elective display access in the week.

Table 6.1.

Likelihood ratio test for the logistic regression terms

\begin{tabular}{llll}
\hline & LR Chisq & Df & $\operatorname{Pr}(>$ Chisq $)$ \\
\cline { 2 - 4 } Manufacturer & 25.75 & 4 & $<0.001 * * *$ \\
Venue Type & 14.3 & 4 & $0.006 * *$ \\
Total hours played & 566.48 & 1 & $<0.001 * * *$ \\
\hline
\end{tabular}

The following graphs illustrate the effect each variable had on the log odds of accessing an elective display at least once. Note \# indicates a significant difference from all other 
levels. The first main effect was the total number of hours played. This was included so that we could test the effect of other variables without the influence of total hours played. Figure 6.3 shows the effect total time spent playing, had on the log odds of accessing an elective display. As expected, there was an upwards trend showing that the more time a machine was used across the week, the higher the log odds of that machine recording one or more instances of elective displays being accessed.

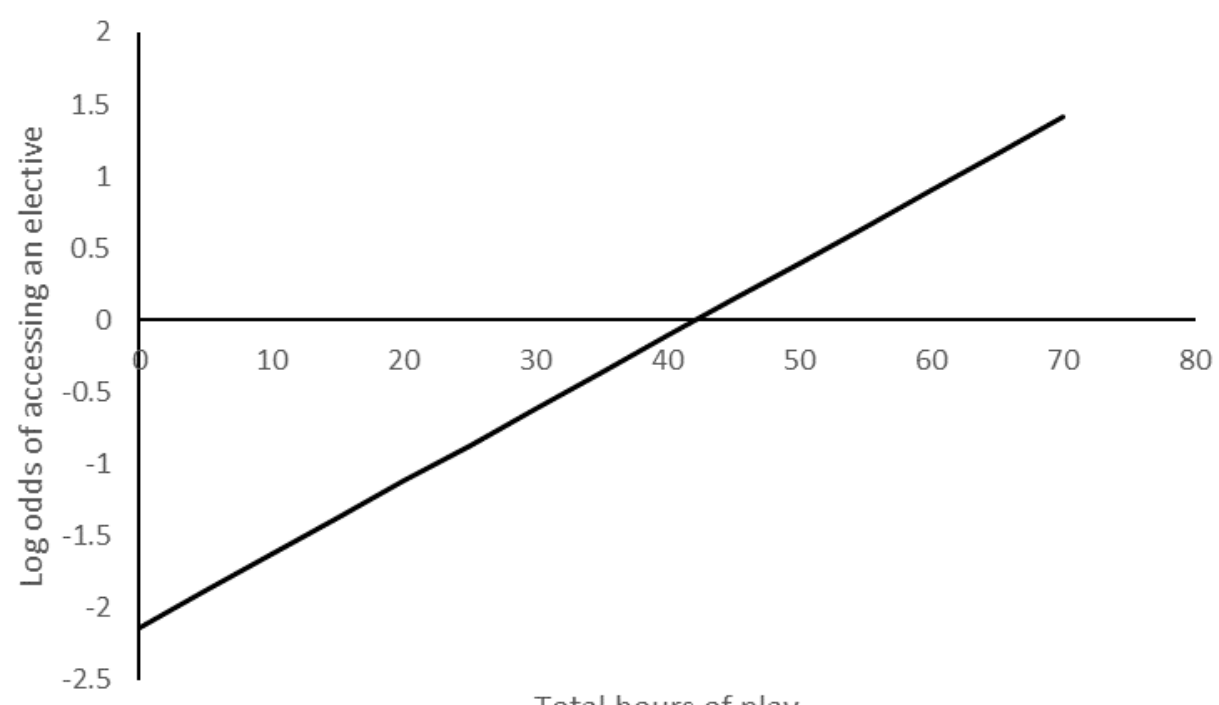

Figure 6.3. Line graph showing the relationship between the total hours played (continuous) and log odds of at least one elective display access.

Figure 6.4 shows that, averaging over all levels of other variables, the likelihood of accessing an elective display differed depending on the venue type in which the machine was being played. There was a greater likelihood of accessing an elective display in a tavern or restaurant when compared to a club. The odds of accessing an elective display in a tavern and a restaurant were 1.40 and 1.61 times the odds of accessing a display in a club, respectively. 


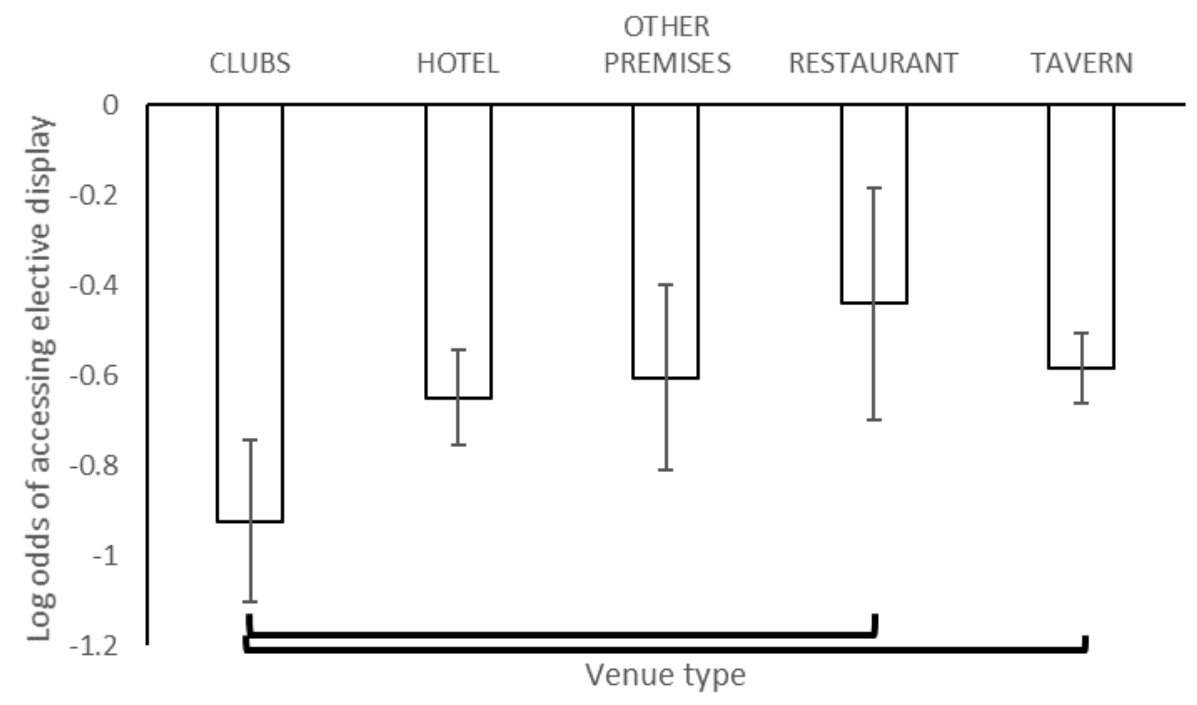

Figure 6.4. Bar graph showing main effect of venue type on the log odds of accessing elective displays across the five venue types. Error bars are $95 \%$ confidence intervals. Statistical indicators are significant to at least the 0.05 level.

The next main effect was for manufacturer as seen in Figure 6.5. This figure shows that the likelihood of accessing an elective display differed based on the manufacturer that developed the EGM. Figure 6.5 shows Star Games machines had a significantly greater likelihood of having the display accessed than machines produced by the other manufacturers. The odds of accessing a display on a Star Games machine was between 1.24 - 1.53 the odds of accessing an elective display on a machine produced by any other manufacturer. 


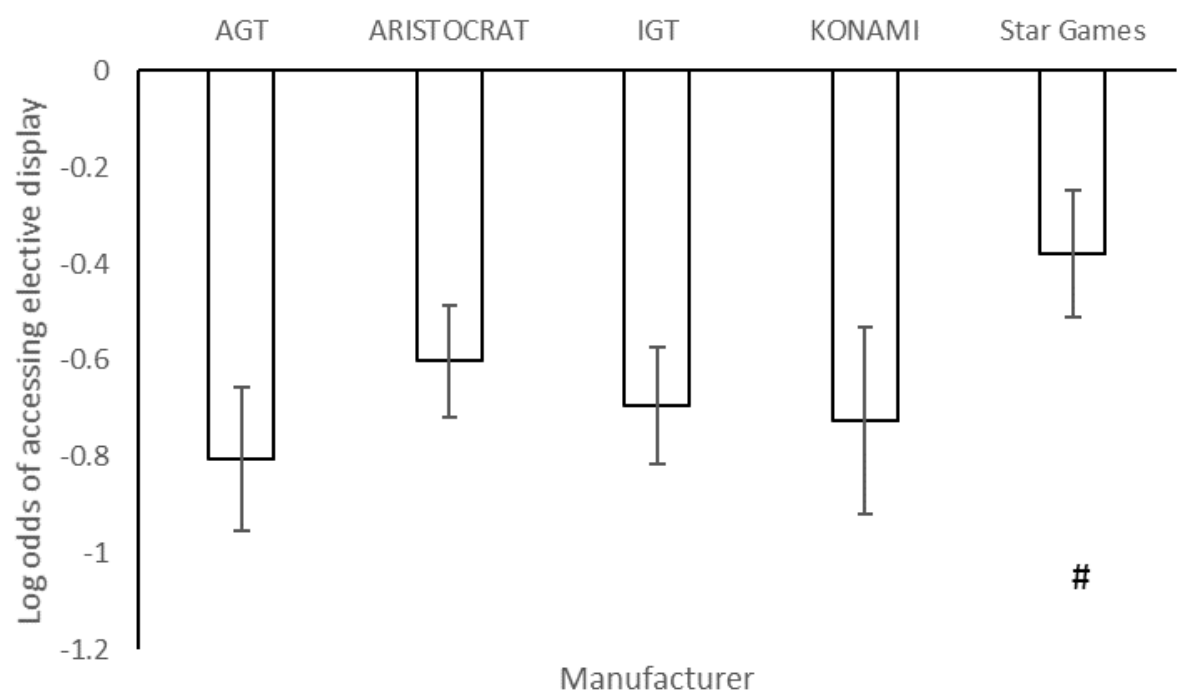

Figure 6.5. Bar graph showing main effect of manufacturer on the log odds of accessing elective displays across the five manufacturers. Error bars are 95\% confidence intervals. Statistical indicators are significant to at least the 0.05 level.

Table 6.2 shows the confusion matrix for predictions made on the training dataset. The matrix probability threshold was set to 0.5 for classifying 'access' responses, against 'did not access' responses. Varying the threshold did not improve the model's accuracy. The model could accurately differentiate between the outcomes of an elective display being accessed versus not at all. Overall, the model accurately classified $68.67 \%$ of observations with higher specificity than sensitivity. It was more accurate at correctly rejecting non-events than correctly accepting real events.

We also ran the same validity check on the test dataset (the remaining $25 \%$ ) in order to see how the final model performed on data it had not seen. Table 6.3 shows the confusion matrix for these data. It shows a very similar pattern of accuracy to that for the training dataset, with overall $67.06 \%$ of observations correctly classified and again higher specificity than sensitivity. The model's performance on the test data was almost exactly the same as its performance on the training data. This therefore showed this 
model was suitable for predicting whether or not an elective display was accessed on data that it had not seen.

Overall, from the data we had access to, venue type, manufacturer and amount of time spent playing were sufficient to, with some accuracy, determine whether or not a machine recorded one or more elective displays in a week. This is useful when identifying ways to make the displays more useful and determine why they are accessed at such a low rate.

Table 6.2

Confusion Matrix for training dataset

Observed

$\begin{array}{lll}\text { Predicted } & \text { No Access } & \text { Accessed }\end{array}$

\begin{tabular}{lrrr}
\hline No Access & $3817(58 \%)$ & $1649(25 \%)$ & 5466 \\
Accessed & $428(6 \%)$ & $736(11 \%)$ & 1164
\end{tabular}


Table 6.3

Confusion Matrix for test dataset

Observed

Predicted $\quad$ No Access Accessed

$\begin{array}{llll}\text { No Access } & 1226(55 \%) & 579(26 \%) & 1805\end{array}$

Accessed

$149(7 \%) \quad 256(12 \%)$

405

$1375 \quad 835$

\section{Multiple regression}

The second part of the analysis investigated whether we could predict the number of times an elective display would be accessed on a particular machine given that an elective display had been accessed at least once. The same dataset used in the logistic regression above was used for the multiple regression. It included all the same predictor variables of manufacturer, venue type, region and time spent playing. However, the key outcome variable differed. Instead of a binary coded outcome, a continuous outcome was used; how often the elective displays were accessed on a machine. As the likelihood of accessing an information display increased with amount of time the machine had been played for, rather than looking at the absolute number accessed per week, we divided this number by the total number of hours played per week creating a rate of electives per hour.

Once we had removed the sessions with 0 button presses (dealt with in the logistic regression above) and log-transformed the data, we achieved a normal 
distribution, with a normal distribution of the residuals also. We assessed this visually using a Q-Q plot.

Like Study 2 and the logistic regression above, we started with the most complex version of the model and conducted a backwards selection process using AIC values. The most complex model was a three-way interaction between manufacturer, region and venue type. Following the backwards selection process the remaining terms identified as contributors to the outcome variable were two interactions: that between venue type and region, and between venue type and manufacturer. The $\mathrm{R}^{2}$ value was 0.1137 .

Table 6.4 shows the results of a likelihood ratio test. As expected, the two key interactions remaining in the final model following the backwards selection process, were significant contributors to the outcome variable of electives per hour. The interpretation of results will focus on these two interactions. We examined how the manufacturer's influence on the rate of elective displays accessed differed by venue type, and how the venue type's influence on the rate of elective displays accessed, differed by region. 
Table 6.4

Likelihood ratio test for final linear model terms

\begin{tabular}{lcccc}
\hline & Sum Sq & Df & F value & $\operatorname{Pr}(>\mathrm{F})$ \\
\cline { 2 - 5 } (Intercept) & 95.87 & 1 & 164.1511 & $<0.001^{* * *}$ \\
Manufacturer & 33.70 & 4 & 14.4254 & $<0.001^{* * *}$ \\
Venue Type & 8.34 & 4 & 3.5684 & $0.007^{* *}$ \\
Region & 4.36 & 2 & 3.7289 & $0.024 *$ \\
Venue Type:manufacturer & 23.72 & 16 & 2.5383 & $<0.001^{* * *}$ \\
Venue Type:Region & 10.70 & 8 & 2.2901 & $0.019 \mathrm{~s} *$ \\
& & & & \\
\hline
\end{tabular}

The graphs below display the two significant interactions. The first significant interaction was that between venue type and manufacturer. Figure 6.6 shows this interaction through two graphs, each displaying one side of the interaction. The top graph in Figure 6.6 shows that, for AGT machines, there was a significant difference in the log of electives per hour between other premises and both: hotel and tavern. For Konami machines, there was a difference between clubs and: hotels, restaurants and taverns. There were however, no differences across venue types for the remaining manufacturers: Aristocrat; IGT; and Star Games. Looking at the same data again but the opposite side of the interaction we can see differences between manufacturers within each venue type. These differences found between the manufacturers were contingent on the venue type, no differences were consistent across all. 


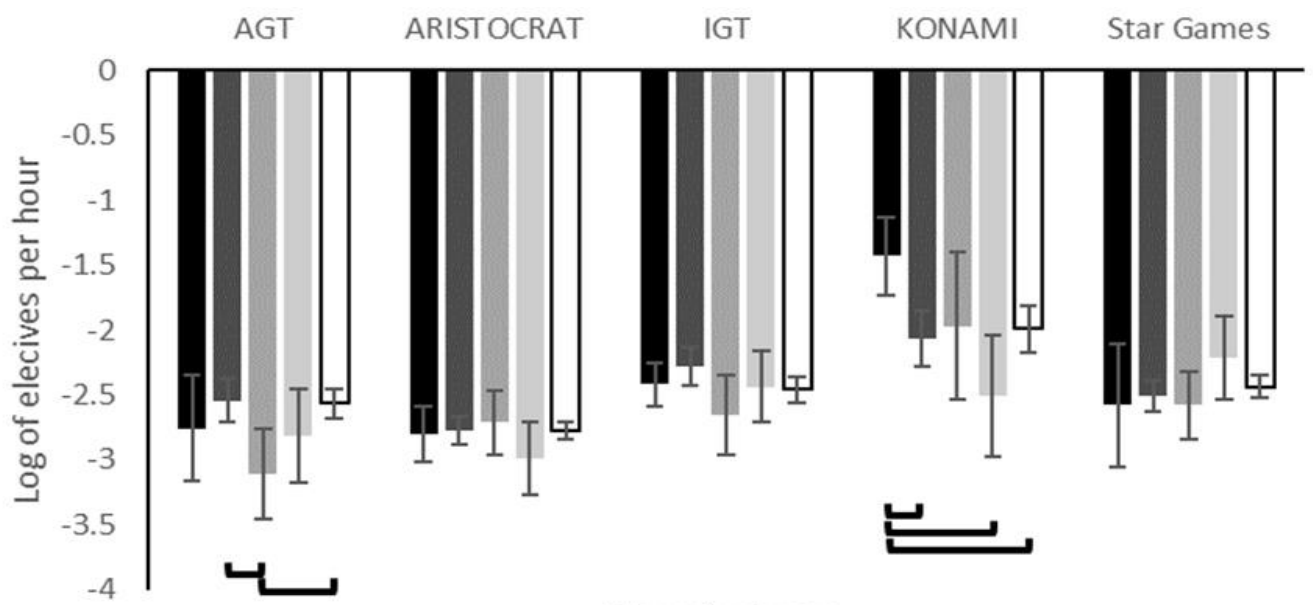

Manufacturers

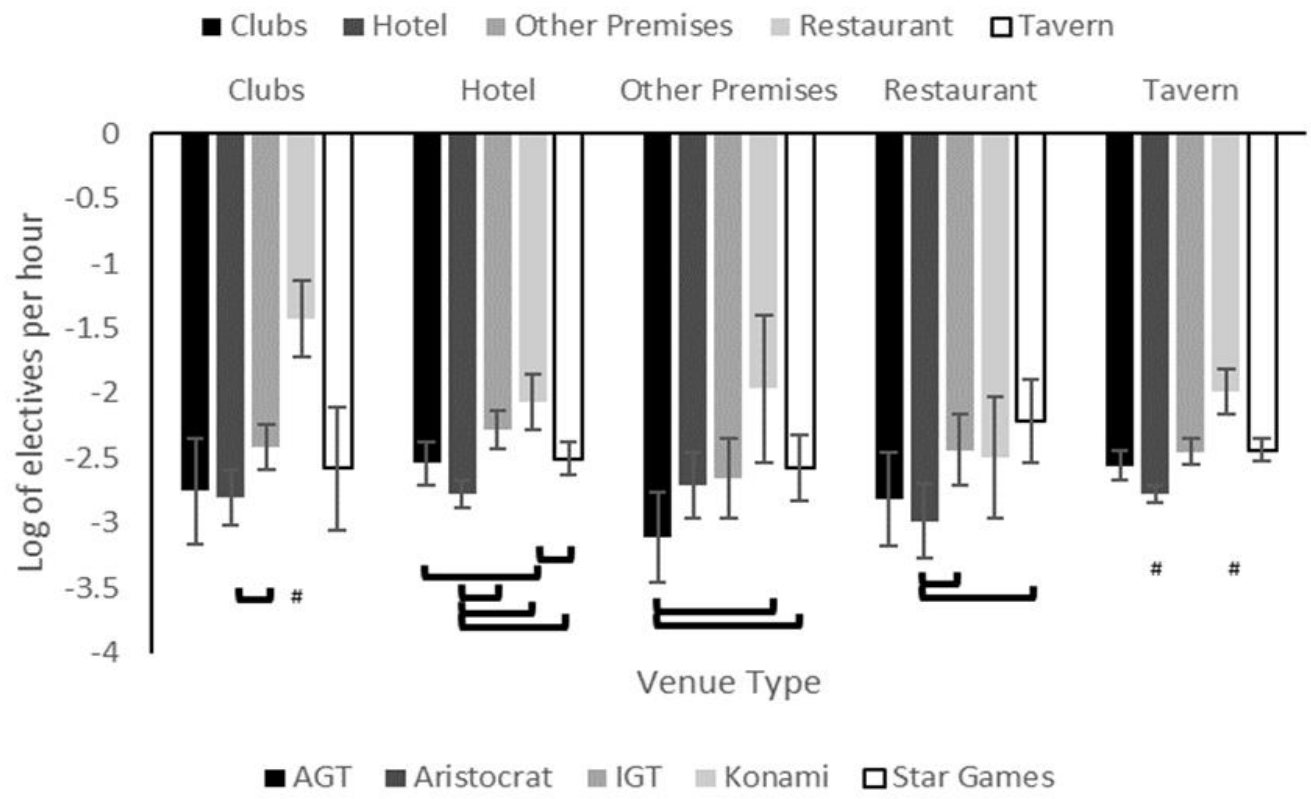

Figure 6.6. Two bar graphs showing the interaction between venue type and manufacturer. Bars indicate the log of the electives per hour. The top graph shows one side of the interaction with differences in log of electives between venue types shown for each manufacturer. The bottom graph shows the same data repeated displaying the opposite side of the interaction. This shows how the log of electives per hour differ between the manufacturer across each venue type. Error bars are $95 \%$ confidence intervals. Statistical indicators are significant to at least the 0.05 level. 
The second significant interaction was that between venue type and region and is presented in two ways in Figure 6.7. The top graph shows that for clubs there was a significant difference between the Central and Northern regions and that for taverns there was a significant difference between Northern and both: Central and Southern. The other three venue types: hotels; other premises; and restaurants, showed no difference across the regions. The bottom graph shows the opposite side of the interaction, the difference between venue types across the three regions. Only the Northern region showed a difference across the venue types with clubs having a higher log rate of electives being pressed per hour than all other venue types. 


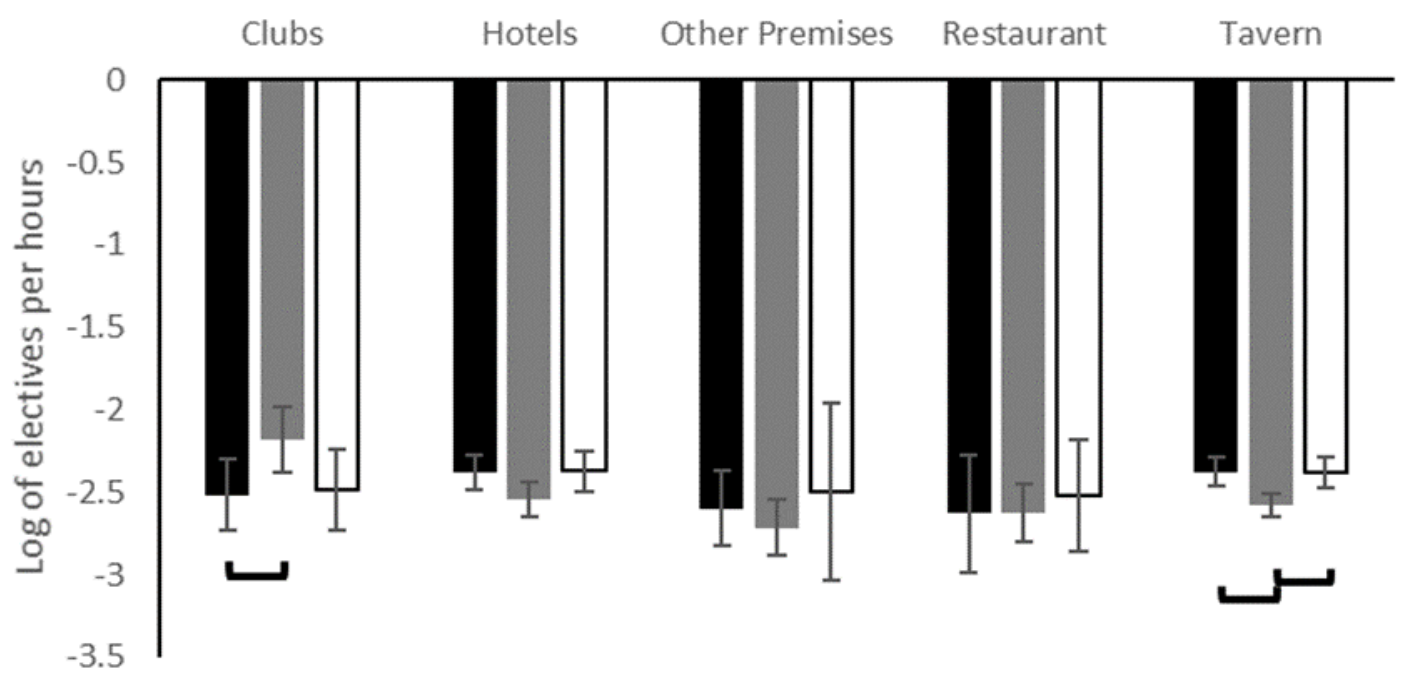

Venue Types

- Central $=$ Northern $\square$ Southern

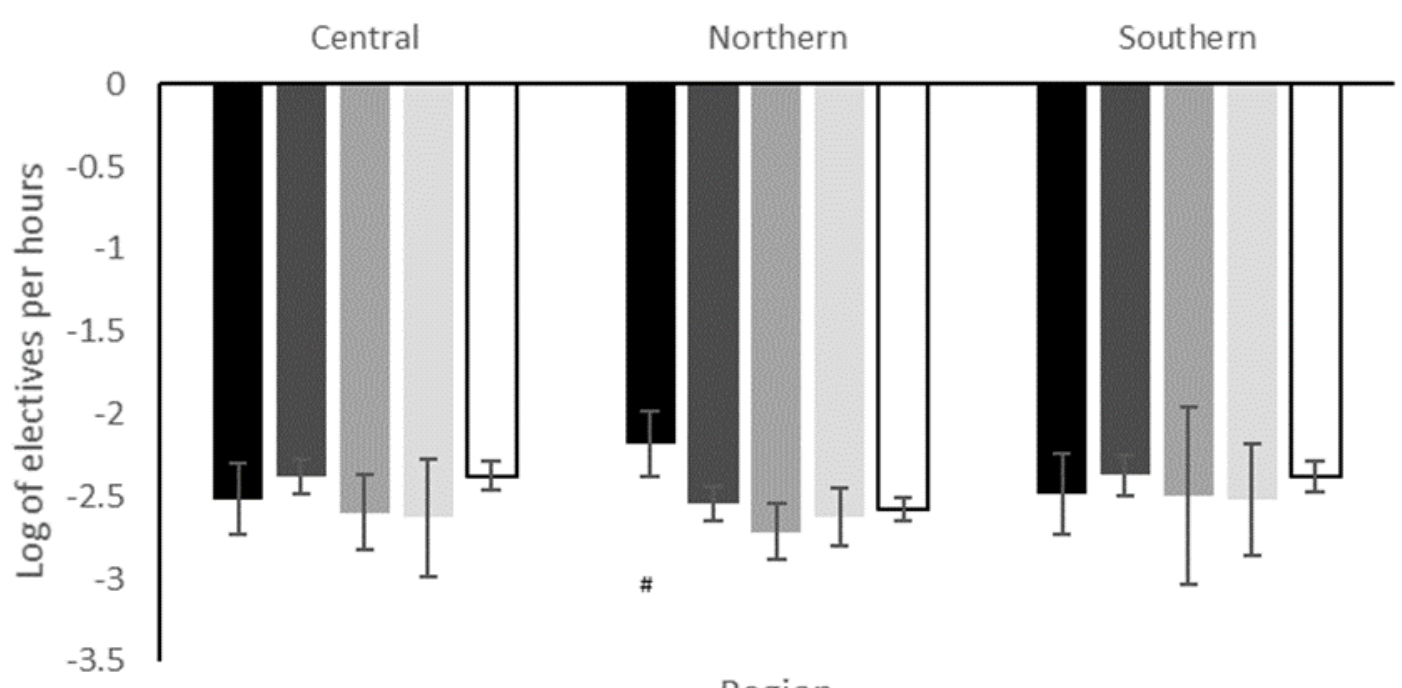

Region



Figure 6.7. Bar graphs showing the interaction between venue type and region. Bars indicate the log of the electives per hour. The top graph shows one side of the interaction. This is, how the log of electives per hour differ between the regions, across each venue type. The bottom graph shows the same data repeated, but the other side of the interaction. This graph shows the differences in log of electives per hour between the venue types, across the three regions. Error bars are $95 \%$ confidence intervals. Statistical indicators are significant to at least the 0.05 level. 
The low $\mathrm{R}^{2}$ value indicated that this model did not explain much variance in the $\log$ rate of electives per hour (11.37\%). Like the logistic regression, we wanted to check how this model performed at making predictions about the rate at which electives were accessed. Figure 6.8 shows a scatter plot of the model's predicted elective per hour rates for the training dataset, versus the observed electives per hour for the training dataset (note that rates are not log transformed). If the model accurately predicted the rate of elective accesses, a positive linear relationship would be apparent in Figure 6.8. From Figure 6.8 it is clear this model is poor at predicting the exact rate at which these displays were accessed. The model tended to consistently predict low rates of electives accessed, despite observed rates of access varying. For completeness we also tested the model's performance on the test dataset by plotting the model predictions for the test dataset against the observed test data seen in Figure 6.9. Unsurprisingly this graph showed a similar pattern with the model consistently predicting low rates of access despite variation in the observed rates (again on the non-log scale). Zoomed in sections are included in both Figure 6.8 and 6.9 to verify no linear relationship existed.

Both these figures indicate that, from the variables we had access to, we are able to identify some variables which had a significant influence on the rate of electives being accessed. These however, were not influential enough to make accurate predictions about specific rates of access. Given the lack of initial variables and the complex nature of decision making, choosing to access an elective display is likely influenced by many factors, not measured in a dataset like this, such as personality and financial situation. While we could not predict the number of times an elective would be accessed on a machine during the week, we have provided evidence that interactions 
between the measurable variables of venue type, manufacturer and region do have an influence.

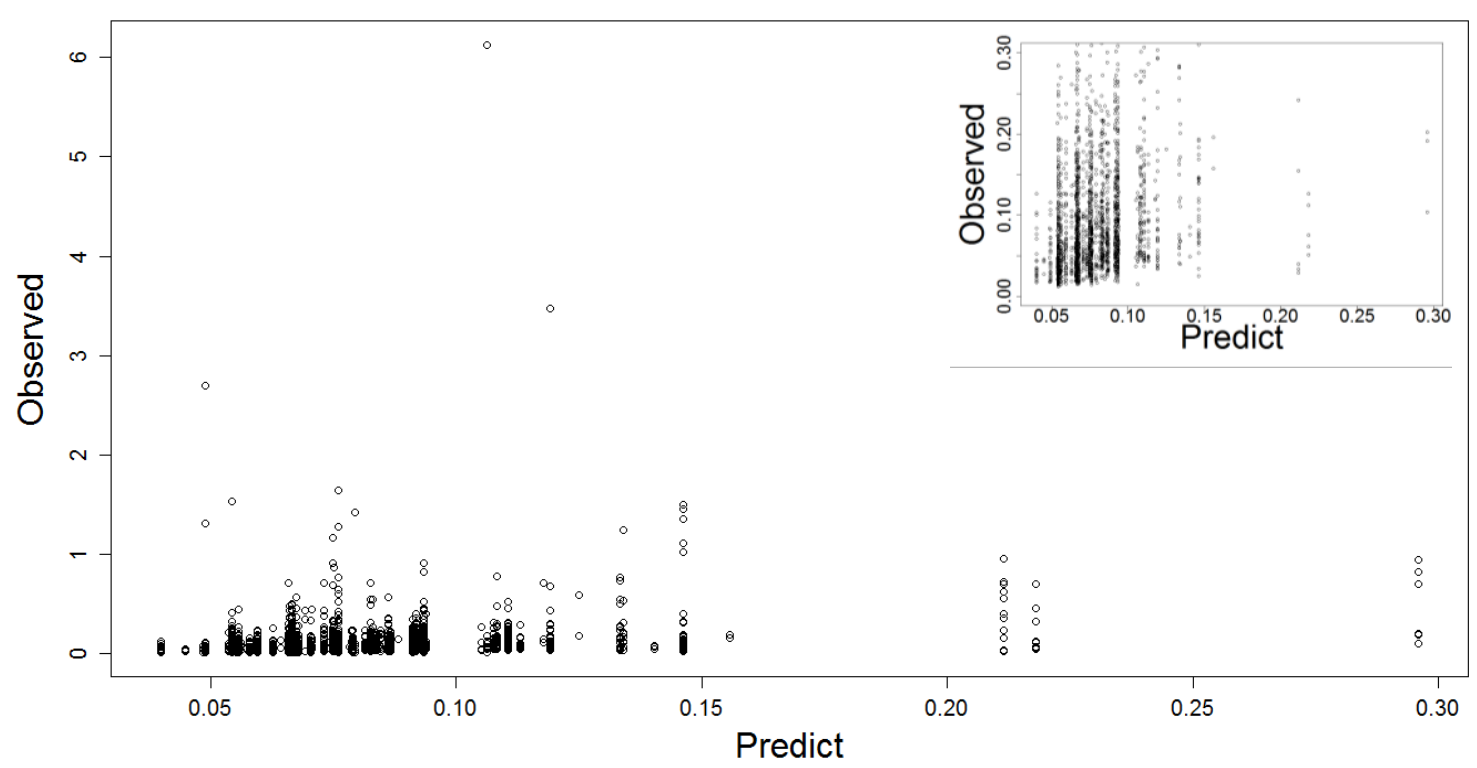

Figure 6.8. Scatterplot showing the predicted and observed elective per hour rates for the training dataset. Note the different scales on the $x$ and $y$ axes. Zoomed in section for clarity shown in the top right corner. 


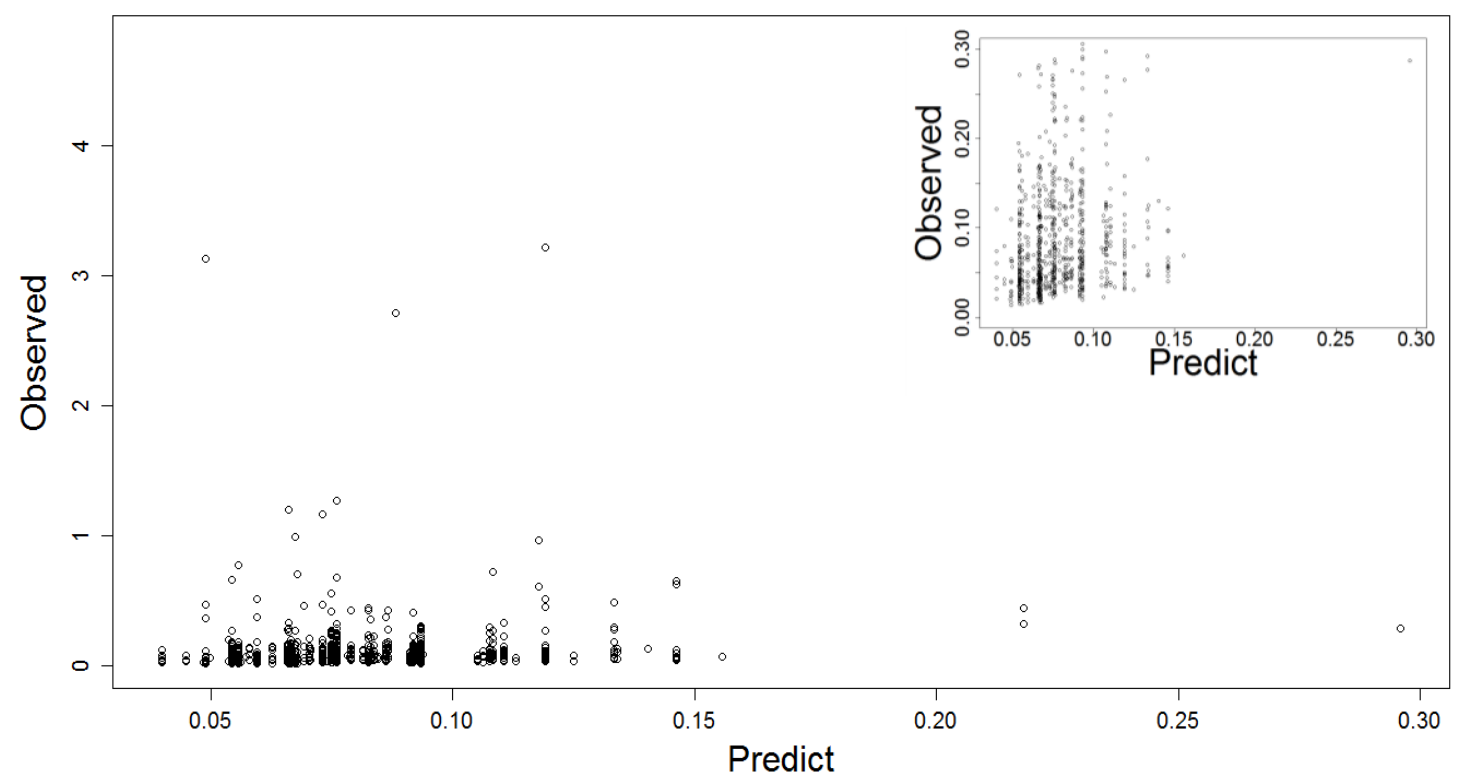

Figure 6.9. Scatterplot showing the predicted and observed elective per hour rates for the test dataset. Note the different scales on the $x$ and $y$ axes. Zoomed in section for clarity shown in the top right corner. 


\section{Chapter 7: General Discussion}

This study covered two forms of information displays. For clarity they will be discussed separately.

\section{Pop-up Information Displays}

Summary. To summarise, we could not find evidence of pop-ups working effectively as a harm minimisation tool, as fewer than $2 \%$ of pop-ups resulted in quitting gambling. Our analysis also revealed differences in how pop-ups were scheduled, indicating manufacturers had interpreted the regulations in different ways. Despite the low quitting rate from pop-ups, we identified a number of complex interactions between machine and session features that influenced how often gamblers use the pop-ups to quit. These machine features were manufacturer and venue type (location of machine), while the session features were number of pop-ups seen, rate of play (dollars per minute), net gain and time of day.

While the overall effect of each variable is hard to isolate and summarise, given the number of interactions, there are some overall conclusions that we can draw. The type of venue where players were gambling, influenced the likelihood of quitting on a pop-up as a main effect. Despite manufacturer interacting with other variables, the manufacturers had a consistent overall pattern of influence. Players were consistently more likely to quit on Star Games machines and least likely to quit on AGT machines.

While the number of pop-ups seen in a session interacted with manufacturer, time of day, net winning and rate of play, the consistent finding was: as more pop-ups were seen, the likelihood of a player quitting on a pop-up reduced. Also, while a player's net winning interacted with a number of variables, they were consistently less likely to quit on a pop-up when at a net loss, compared to a net win. Rate of play also 
interacted with many variables, the general finding being: as rate of play increased, the likelihood of quitting on a pop-up decreased. This being said, many of these effect sizes were very small and when tested, the model performed poorly at differentiating between outcomes. Therefore these results must be understood with this in mind.

Discussion of findings. As New Zealand pop-ups are unique, there was limited previous literature relating closely to the current finding that New Zealand pop-ups are not working effectively to minimise gambling harm. However, Palmer du Preez et al.'s (2014) and Palmer du Preez, Landon, Bellringer, Garrett, and Abbott's (2016) studies are comparable as they also examined pop-ups in New Zealand. Of interest, the percentage of pop-ups resulting in quitting in Palmer du Preez et al.’s (2014) observational research was larger than the percentage found in the current study. Palmer du Preez et al. (2014) found, of the 18 pop-ups observed, two resulted in cashing out and two resulted in changing machines or games, resulting in an overall rate of quitting on a pop-up of $22 \%$. This compares to our finding of $2 \%$ of pop-ups resulting in quitting. As both studies were conducted in New Zealand we would expect pop-ups to have a similar impact on the players in 2014 as they did on the players across New Zealand in the current 2018 study.

This difference could highlight some circumstances in which pop-ups are more effective. Perhaps awareness of being observed within Palmer du Preez et al.'s (2014) study led to an increased likelihood of people quitting following the appearance of a pop-up, as this is the key difference between the Palmer du Preez et al.'s study and the current study. Previous studies have demonstrated the observer effect occurring with gamblers, specifically in relation to reduced time and money spent gambling (Mishra, Morgan, Lalumiere, \& Williams, 2010). Palmer du Preez et al. noted that efforts were made to ensure observers did not impact on naturally occurring gambling behaviour, 
however, it was unclear to what extent this was achieved. The possibility of active observation leading to higher quitting rates would require more investigation, but could prove a useful starting point on how to increase the percentage we found of quitting on a pop-up from $2 \%$. If the feeling of observation and being accountable for one's gambling behaviour leads to people gambling more responsibly, this could be utilized for improving the effectiveness of these interventions. This concept of accountability to someone else relates to Harris and Griffiths' (2017) suggestion that public commitment can increase compliance with a goal.

Palmer du Preez et al. (2016) accompanied their observational study with a survey of players, asking what impact pop-ups would have on their behaviours. They found $6.4 \%$ of participants reported they were likely to stop their gambling session in response to a pop-up, with a further $18.4 \%$ suggesting they were most likely to change machines. Both of these responses would have appeared as quitting on a pop-up in the current study. We therefore combined both responses within this survey, resulting in a percentage close to $25 \%$ of players, much larger than the current $2 \%$. This number was only the players who predicted this behaviour was "likely", they did not predict that they would quit every time they saw a pop-up. It is therefore unsurprising that responses in this survey suggest an inflated rate of quitting compared to that found in the current study.

Our study also identified clear differences in the way manufacturers scheduled pop-ups. IGT, Konami and Star Games scheduled their pop-ups at much more predictable times than AGT and Aristocrat. Linking the differences in predictability between manufacturers, to the differences in how effective pop-ups were revealed an interesting pattern. Manufacturers with predictable pop-ups also displayed the highest rates of quitting on a pop-up. When exploring why this might be, it is possible that pop- 
ups appearing at more predictable times can be more effectively avoided through changing machines or cashing out prior to an expected pop-up.

While this might initially indicate predictable pop-ups might have a reduced effectiveness, it is important to consider the way effectiveness was measured within the current study. To account for repeated exposure to pop-ups we measured effectiveness as the number of pop-ups resulting in quitting as a percentage of the total pop-ups shown. While we could not find any evidence of this in Study 1, it is possible that being able to pre-empt pop-ups would mean players could prevent them from appearing. This would mean machines with predictable pop-ups might have a decreased number of popups appearing in total, than compared to non-predictable pop-ups (all things being equal). If this were the case, the number of pop-ups resulting in a quit response, as a percentage of this lower value would therefore be higher, as found in this study. To examine the possibility of this occurring we looked back to the data in Study 1 comparing the number of pop-ups occurring per hour between the manufacturers. These differences however, did not provide evidence consistent with this explanation above.

Some previous literature found the reduction effect pop-ups had on gambling remained unchanged when the message element of a pop-up was removed. This indicated the break element of a pop-up alone was enough to reduce gambling (Cloutier et al., 2006; Ladouceur \& Sevigny, 2003). Other researchers, however, found the message element of a pop-up necessary for a reduction in gambling to be observed (Jardin \& Wulfert, 2009, 2012).

The current study on New Zealand pop-ups, which have a message and enforce a minimum break of 15 seconds, identified little evidence of pop-ups working effectively. If breaks were driving the harm minimisation effect as suggested by some studies, we 
would expect New Zealand pop-ups to have a notable influence on quitting, while the message would be there, but provide no additional advantage. Given that New Zealand pop-ups had no notable effect on gambling, this indicates that neither the presence of a break, nor the presence of a message were working effectively to have a harm minimisation effect.

The large time gaps between New Zealand pop-ups (up to 30 minutes), indicated a state of dissociation could be reached between pop-ups. Our findings therefore could not be explained in line with the previous explanation that breaks were not effective due to the short time between pop-ups preventing a state of dissociation being entered into. The additional finding that messages displayed on New Zealand pop-ups were not effective, may be unsurprising given past research and suggests that alterations to message content might be a way to enhance pop-up effectiveness (see below).

Possible improvements. The findings of our logistic regression indicated that venue type had a significant influence on the likelihood of someone quitting on a popup. There tended to be a greater likelihood of people quitting in commercial premises such as restaurants, compared to non-commercial premises such as RSA clubs. This suggested key differences in the way harm minimisation tools are utilised in different venues. There were a number of possibilities as to what could be driving this. A review investigating the influence gambling venues had on gambling behaviour, identified features such as opening hours, visibility of clocks, lighting and availability of ATMs nearby, as possible venue features that could influence behaviour (Thomas, Mora, \& Rive, 2010).

Another possible factor that varied between venues and could explain the current findings was the number of other machines available in the venue. Sagoe, Pallesen, 
Griffiths, Mentzoni, and Leino (2018) found evidence of this with people spending more time and money in smaller venues with 2-5 machines compared to all other machine number categories. This could potentially provide an explanation for the current findings. Non-commercial premises like clubs tend to have fewer EGMs than commercial venues such as restaurants (Department of Internal Affairs, 2018). It is plausible that in venues with fewer machines, people have an incentive to remain playing at a machine so as to not surrender it to another player. In the current findings, players in non-commercial premises with fewer machines could have been less persuaded to quit by a pop-up appearing, as they did not want to give up the machine they were playing on.

Another significant influencer we identified was manufacturer. Despite interactions, we found the likelihood of quitting on pop-ups differed between manufacturers in a consistent way. Players on Star Games machines were most likely to quit on a pop-up followed by IGT, then Konami, then Aristocrat, then AGT. Previous research by Hammond (2011) identified features on warning labels such as contrasting colours, using boxes to frame words, size of font, graphics and layout of information, all influenced the effectiveness of the warning. Under the current regulations almost all these features are at the discretion of the manufacturers (Department of Internal Affairs, 2016). It is therefore unsurprising the differences that we have found, exist. It is possible the combination of features on Star Games machines are responsible for them having the highest rate of quitting on a pop-up.

While the current study analysed a dataset collected from real EGMs in New Zealand, experimental study designs can complement this research and provide answers to other questions. Experimental studies can be conducted in controlled laboratory settings using simulation EGMs, where the experimenter can manipulate one small 
feature while holding everything else constant. With this careful control researchers can determine the effect of individual features. A logical next step would be to analyse popup displays created by each manufacturer in an experimental setting to determine the possible causes of the differences in quitting rates. The possible approach to designing this experimental study would be to identify differences among manufacturers' displays and test the impact of them experimentally. We repeatedly contacted manufacturers of New Zealand EGMs to conduct this analysis, however, not all manufacturers responded with examples of their pop-up displays. If this research was possible and particular features associated with effectiveness were identified, regulations regarding the format in which the information needs to be displayed, could be established. Alternatively, the most optimal screen could be developed and this display could be a requirement for all manufacturers.

The variable of net winning was also identified as a key influencing variable and, despite interactions, sessions ending on a net loss consistently resulted in a reduced likelihood of quitting on a pop-up compared to those ending with a net win. This was concerning for those wanting to minimise harm as it indicated that players who had lost their money were the least likely to use the harm minimisation tool. This finding is consistent with the idea that people chase their losses, those who were already losing were the ones more likely to persist at playing. Chasing losses is the persistent engagement with EGMs (spending additional money), with the aim of recouping previous losses (Breen \& Zuckerman, 1999). It is one of the central characteristics of problem gambling (Campbell-Meiklejohn, Woolrich, Passingham, \& Rogers, 2008), implicating loss chasing behaviour in harmful gambling.

The current finding that people losing are less likely to quit on a pop-up, emphasises the possibility that these displays cannot target players chasing losses 
(possibly high risk players). Harm minimisation tools are generally designed to have the greatest effect on high risk players while having little to no effect on recreational gamblers (Gainsbury \& Blaszczynski, 2012). This would suggest the small amount popups are used, might not even be by the target problem gambling group. This was a useful finding in terms of working out ways to improve the displays as increasing the rate they are used might not be useful if they are not effective for players at risk of harm.

Another key variable that influenced the likelihood of someone quitting on a pop-up was a player's rate of play. As the number of dollars spent per minute increased, the likelihood of quitting on a pop-up was reduced. A higher spend per minute might reflect gamblers making larger bets and/or more bets per minute. Griffiths (1994) found the more often people gamble, the higher their rate of play. This suggests players more likely to be at risk of harm, were the players with a reduced likelihood of quitting on a pop-up. This is problematic as fast play can perpetuate gambling issues by reducing a player's ability to pause and think about what they are doing (Harris \& Griffiths, 2018). Harris and Griffiths also supported this concept, finding fast play speeds were appealing to problem gamblers, more so than other gamblers. It is useful to know there is further evidence that players most associated with problematic gambling patterns are those least likely to use pop-ups.

There was one exception to this overall trend, which was machines manufactured by Konami. Unlike the other four manufacturers, where, as rate of play increased the likelihood of quitting on a pop-up reduced, Konami machines displayed a flipped trend. The likelihood of quitting on a pop-up increased as rate of play increased. This could be an indication that Konami pop-ups were specifically effective for players with high rates of play (high-risk players). 
There could be a place for further experimental study where display features between the manufacturers are compared for effectiveness. Not only would effective features need to be identified in the general population, but it would be useful to conduct this experiment on players with differing levels of problem gambling severity. This would identify features most effective for the target group. It is important to be aware that despite this striking opposite trend, the absolute size of differences between Konami and the other manufacturers remained small and might not have a strong effect in applied settings.

Despite identifying variables that significantly influenced players' likelihood of quitting, these effects were small in magnitude and, when using the model to predict quitting behaviour, no combination of predictor values in the dataset predicted that a session was more likely than not to end with a quit on a pop-up. This finding was most likely driven by the fact that the event of quitting on a pop-up was rare, however, it also highlighted the fact that, despite finding significant influencers, these are far from the complete set of variables responsible for a player quitting on a pop-up. This knowledge informs future research; these variables are good starting points but additional variables would need to be investigated in order to make a meaningful improvement to New Zealand pop-ups.

When discussing how to make pop-ups more effective, it is important to refer back to the original reasons the Cabinet Paper cited for the inclusion of pop-ups (Cabinet Policy Committee, 2003). The Cabinet Paper cited two studies indicating the need for information displays (Department of Internal Affairs, 2001, 2005), but it appears these current pop-up displays do not address the reasons for their initial inclusion or achieve the intended goal. Addressing people's incorrect beliefs about gambling and lack of knowledge about EGMs were identified as the reason pop-ups 
were to be required in the regulations (Department of Internal Affairs, 2005). The current pop-ups provided feedback about play but did not appear to correct false beliefs. It is possible the feedback displayed on pop-ups could improve awareness of losses, but it remains unclear whether false beliefs are improved by the current pop-ups. The Department of Internal Affairs (2001) provided evidence that people predicted that if they were told 'the house always wins', this would help them to reduce their gambling. However, this information is not included in the current pop-ups. It appears that some of the reasons for including these pop-ups in the first place were not incorporated into regulations that dictate what the pop-ups need to contain.

Including explicit messages to reduce the false beliefs is one way pop-ups could be improved from their current design, making them more in line with the original reasons for their implementation. While the literature was mixed, there was evidence of pop-ups targeting false beliefs, resulting in gambling reduction (Benhsain et al., 2004; Jardin \& Wulfert, 2009, 2012). Given the current pop-ups are not effective, New Zealand could include similar messages targeting false beliefs as a possible improvement. It should however be noted, that the effect these messages had on reducing gambling in the studies above appeared contingent on an exposure rate that might not be possible in New Zealand. As noted in the introduction, these types of popups were only effective when shown to players at high frequencies. The frequency New Zealand pop-up displays appear is likely to be limited to a range agreed upon by both the government and the manufacturers. Agreement to increase this frequency by the gambling industry might be impossible to achieve, making these messages a less viable option.

Findings of the current study, and findings of previous studies appear to be at odds. Previous literature indicated the more frequently pop-ups appeared, the more 
effective they became (Cloutier et al., 2006; Jardin \& Wulfert, 2009). The current finding within the logistic regression indicated each additional pop-up reduced the likelihood of quitting. The content types of the pop-ups differed between studies, however, it is interesting to explore this contrasting finding between our study and previous literature.

One way to explain this difference is perhaps the effect found in the literature is not driven by the cumulative number of pop-ups that appear, but is purely driven by the short interval time between pop-ups. If this was the case cumulatively displaying more and more pop-ups but with long intervals between will have no greater effect than just one. This is observed in the current study. The time between pop-ups remains lengthy (up to 30 minutes), and no advantage of additional pop-ups appearing was found. This finding can also be understood by persuadable players being more likely to quit on the first pop-up, while less persuadable plays who do not intend to quit in response to a pop-up being unlikely to change their stance after seeing more pop-ups.

Pre-set limits are another option for improving the currently ineffective pop-ups as this was one of the more promising pop-up content options within the literature (Auer \& Griffiths, 2013; Auer et al., 2018; Kim et al., 2014; Stewart \& Wohl, 2013; Wohl et al., 2013). These messages tell a player that their spending has reached a limit they previously set as their maximum. These pre-set limit reminders were more effective than pop-ups showing information about current session totals (Auer \& Griffiths, 2015; Auer et al., 2014; Schellink \& Schrans, 2002; Wynne \& Stinchfield, 2004). One could think combining multiple pieces of information would make these pop-ups more effective however, this does not appear to be the case in Hollingshead, Wohl, and Santesso's (2019) study. They found no advantage of having personalized feedback 
alongside the pre-set limit reminder. Pre-set limits are a potential way in which New Zealand pop-ups could be modified to increase their effectiveness.

It is useful to note the manipulation check included within Hollingshead et al.'s (2019) study could provide an explanation for why the current study has discovered pop-ups only rarely result in quitting. Their manipulation check tested the ability of players to recall what the pop-up generally contained information about. They found that a large number of people failed this check, and this persisted when they made the pop-ups stay on the screen for a minimum of 10 seconds. This indicated that pop-ups of 10 second duration are not read in the first place and therefore the specific content is a secondary issue in making these pop-ups more effective.

This suggestion that the pop-up content was not read would be useful to explore given the findings of the current investigation. Rather than focusing on finding the optimal content, perhaps the pop-ups are not even being attended to in the first place and therefore would benefit from changes making them more appealing to read. Of interest, Palmer du Preez et al. (2016) found that 57\% of people were aware of pop-up messages and $38 \%$ of people indicate that they saw them often. Half of these people reported they would be likely to read the content. In the observational part of the study they found that indeed over 55\% of people did appear to read the content, $33 \%$ watched other gamblers and the final $11 \%$ displayed frustration. The findings from New Zealand therefore indicate that many people attend to pop-up information, but future studies could thoroughly disentangle these two possibilities, to determine what needs to be targeted first: content, or attractiveness to read, when attempting to improve the ability of pop-ups to encourage responsible gambling. 


\section{Elective Information Displays}

Summary. The second part of the thesis focused on elective information displays; the other type of information displays found on New Zealand EGMs. In summary, there is also little evidence of elective displays being an effective harm minimisation tool. Two further studies were conducted to identify what variables influenced when elective displays were accessed by players. The first investigation determined that manufacturer and venue type were key predictors of the binary outcome: accessed versus not accessed. These variables along with session duration, were strong enough to accurately predict elective displays being accessed on a machine from electives not being accessed at all. Secondly, we identified variables influencing how many times elective displays were accessed, given that they had been accessed once. Venue type, region and manufacturer were all key variables, and presented their effect in the form of two interactions. The effect manufacturer had on the rate of elective display accessed, differed by venue type and the effect venue type had on the rate of elective displays accessed, differed by region. While these interactions were identified as having a significant influence on the number of times an elective was accessed, they were not strong enough to accurately predict actual access rates.

Across New Zealand, 0.0398 elective displays were accessed on average per hour of total play. This indicated they were unlikely to be working effectively at reducing gambling harm. The key piece of comparable research is that conducted by Palmer du Preez et al. (2014). They indicated that $69 \%$ of people were aware of elective displays and $43 \%$ indicated they had used them to quit previously (rarely to often). Because Palmer du Preez et al.'s measures were not about consistent use, it was not possible to assess whether the rate of access per hour we identified, was in the expected range or not. Forty-three percent of players indicating they have used elective displays 
appeared high in comparison to the low rate of access we found. However, this was entirely possible given we observed access over a single week whereas Palmer du Preez et al.'s question was about use at any previous time.

The low rate at which elective displays were accessed had interesting implications when returning to the original reason for their inclusion. It is likely that these displays are what the Cabinet Paper was referring to when aiming to reduce player's false beliefs, as these displays contain factual information about play such as the RTP rate (Cabinet Policy Committee, 2003). False-belief-correcting messages tended to only be effective at influencing gambling when presented at a high frequency (Benhsain et al., 2004; Jardin \& Wulfert, 2009, 2012). Given we have discovered this low rate of access to elective displays it is indicative that these displays may not be able to achieve their goal of reducing false beliefs.

Because the data was in the form of daily machine totals that were then collapsed across the week, it was unknown whether the multiple presses of the elective display button were the same players, or multiple different players on the same machine throughout the week. It is likely that different factors influence a player to access the display a first time, than the factors influencing the same player to access it repeatedly. Repeated access could be influenced by the content of the display seen at least once before, (or content viewed on other machines previously), however the first access is less likely to be influenced by this. It is more likely to be influenced by factors such as the placement of the information button providing access to the display. It is possible however, that accessing only once in the dataset is also influenced by having seen an elective display on a previous session prior to the week studied. Having access to data that tracked players, rather than machine totals, would allow an investigation into single 
versus repeated access and allow more refined conclusions to be drawn about how these electives are used by players as a harm minimisation tool.

Possible improvements. Once observing elective displays were accessed at such a low rate, we tried to identify how to make them more effective. We first investigated what factors influenced whether electives were accessed at all.

We first identified manufacturer as a key predictor of access to electives. As manufacturers have freedom with the design of their machines, identifying differences between machines would be a useful place to start when aiming to increase the number of players accessing these displays. As this part of the analysis aimed to predict access versus no access to electives, one factor to investigate would be differences in the positioning of the information button. Hammond (2011) identified size, colour, contrast and many other features as important features of making warning labels effective. It is therefore likely that these factors, alongside the placement of the button have an influence on how people engage with these information buttons. Comparing between manufacturers to find the optimal way in which to present the information button would be useful when aiming to increase player's elective access to session and machine information. Player's previous exposure to displays could also have impacted their likelihood to access a display versus not at all, making the displays themselves a factor to also investigate. It is also interesting to note that the pattern of how often electives were accessed across manufacturers was similar to the pattern of how often pop-ups resulted in quitting. AGT had the lowest rate of electives accessed and the lowest percentage of pop-ups resulting in quitting. This indicated there could be consistent factors about AGT machines and their displays that appear to have slightly less harm minimisation potential than other machines. 
Venue type was also an important variable for predicting when the electives were accessed. Factors such as layout of the gaming machines (whether they are in a separate lounge or in the main pub/club area), number of other machines in the venue, interactions with others, the overall purpose of the venue, noise, and lighting have all been identified as venue characteristics likely to influence gambling behaviours (Thomas et al., 2010). While it is not immediately obvious why such features would influence the rate of access to electives displays, speculation can be made. Palmer du Preez et al. (2014) note that one reason gamblers dislike pop-ups, is because they breach privacy of players by displaying information about a player's session where other people could view it. If people avoid viewing these elective displays for similar reasons, factors such as layout of venue, number of other machines, purpose of the venue and interactions with other players could all influence the likelihood of a player viewing an elective display.

Using these two variables (manufacturer and venue type) along with session length we were able to differentiate with some accuracy between when an elective display would be accessed during the week versus not accessed at all. This was in spite of the small number of predictor variables available and the event being rare. This indicated these variables are fairly strong influencers of access to elective displays, making them a useful starting point when trying to increase access to them. These variables alone however, could not accurately differentiate all responses, indicating there were other variables (which we did not have access to) that play a significant role in determining when a player will access a display.

The second part of the analysis aimed to predict how many times displays were accessed, given that they had been accessed at least once. This part of the analysis therefore included repeat access to elective displays. Access would not only be 
influenced by the information button but also more likely to be influenced by previous exposure to an elective display on a particular machine (note that we did not have refined information about whether repeated access was by the same player). The number of times an elective was accessed was significantly influenced by an interaction between manufacturer and venue type and an interaction between venue type and region. For all three variables; manufacturer, region and venue type, there was no consistent effect of each. The way each variable influenced the number of times an elective was accessed was contingent on the other variable it interacted with. This complex relationship indicated that if regulators wanted to increase the number of times these elective displays are accessed they would need to be aware there is not one region, venue type or manufacturer that is consistently more effective than others.

In contrast to predicting elective display access versus non-access outcomes, the variables identified as influencing the number of times a display was assessed were not strong enough to accurately predict how often displays were actually accessed. This indicated that manufacturer, venue type and region are far from the complete set of variables which determine the number of times these displays are used.

One clear constraint of the current study is not having access to information about how elective displays influenced play. In order for elective displays to have a harm minimisation effect, their presence would need to increase responsible gambling. Focus group research by Palmer du Preez et al. (2014) indicated that electives might have the opposite effect to that intended and actually increase people's distorted cognitions about gambling. However, given elective displays are accessed at such a low rate, whatever effect they have is confined to a very low majority of players. A next step in the research into these displays would be to try to obtain session level data to determine the effect accessing an elective display screen, had on play. If access to an 
elective tended to decrease time until quitting compared to those who had not accessed a display, it would indicate the content is working in a way to encourage responsible gambling. However, if it increased time until quitting, electives could be having the opposite effect to that intended. This type of further study could then comment on whether solicited advice, as literature suggests, is more effective than information that is given without request (Fitzsimons \& Lehmann, 2004; Van Swol et al., 2017).

A question that comes from the findings of this study was: what is the purpose of these elective displays? The regulation requirements for elective displays do not appear to be designed with the specific intention of encouraging quitting. The information provided is very complex, displaying the chances of winning on various combinations of symbols, maximum and minimum bets and much more. The complexity and amount of information accompanied by poor colour choices and small fonts could confuse a player more than inform them about the risks of continuing play. In addition, despite containing factual information thought to reduce false beliefs, they do not contain false-belief-correcting messages like other studies with the same falsebelief-correcting aim do. Pop-up information displays have a prompt question asking if the player would like to quit, giving an indication that these pop-up displays are intended to assist making decisions to quit. However, it is unclear as to what the key purpose of elective displays are (the other information displays), from viewing their content.

If the intended aim of these elective displays was similar to the aim of pop-ups which was to help people make responsible choices about their gambling, then perhaps these displays could contain similar information. Like pop-up displays, having a combination of information about their session, the game being played and asking a question about quitting would be useful. Perhaps incorporating a self-appraisal element 
within the electives would also be a good way to change these electives to give them more of a harm minimisation intention.

\section{Limitations}

The current study had a number of limitations that must be acknowledged. Firstly, the nature of the dataset we worked with meant there were limited questions we could answer with confidence. We did not have data on a baseline of machines that did not have pop-ups that we could compare to machines with pop-ups, which limited the conclusions we could draw about how pop-ups function.

Secondly, while we were able to use data from real world EGM machines we were also restricted to variables recorded for regulatory purposes rather than ones directly designed with the research question in mind. As seen in the pop-up display logistic regression, the variables available, were not strong enough in combination to predict players' behaviour. Perhaps if we were able to investigate more variables known to influence peoples gambling such as machine features (Taylor, Macaskill, \& Hunt, 2017) and personal characteristics such as problem gambling severity (Productivity Commission, 2010), we would be able to accurately differentiate this behaviour. We could then test if there are optimal circumstances in which these messages are more effective. This could be an area for future research.

If we had the freedom to choose what variables in EGM play were recorded, it would be interesting to consider how pop-ups influence play at the spin level. Having access to this would allow researchers to more fully explore the influence pop-ups have both directly and distally. Being able to look at how people play prior to a pop-up and after, researchers could capture more than just quitting responses. Changes in play characteristics such as play speed, wager amount, and lines bet on could be investigated 
and linked to elective display access. This could also verify the anecdotal evidence Palmer du Preez et al. (2014) found, that people finish their credits loaded onto the machine before quitting after seeing a pop-up.

Another key limitation of the dataset we used and the way that gambling is conducted in New Zealand, is that only sessions can be tracked rather than players. This has its own issues for harm minimisation, in that people with gambling issues cannot be identified, but in the case of this study, it provided a research limitation. Only being able to track sessions, means that we were not aware of sessions conducted by the same person. This meant we could not control for individual player behaviour. This is important when looking at rare behaviours such as quitting on a pop-up, or accessing an elective. It would be useful to know if the occurrence of these behaviours was randomly spread across a wide range of players across the week or if the behaviour was limited to a smaller group of players that conducted these behaviours repeatedly. The Productivity Commission (2010) reported that $15 \%$ of regular players meet the classification for problem gambling, but contributed to $40 \%$ of the spending on EGM. Given this, it would be valuable to determine if a small group of players are the ones engaging with these harm minimisation tools. It is possible low risk people are engaging with these pop-up and elective displays repeatedly, while the high-risk people are not at all. Having access to this data would be beneficial for making more complete conclusions about information display utilisation. This type of individual tracking, rather than sessions is used in Norway and provides excellent data for researchers to explore (Leino et al., 2016).

Another limitation that we faced was only having access to data from machines that ran on QCOM version 1.6.3 technology or newer versions. This was only about half of the machines across New Zealand. It was therefore unknown how pop-ups and 
electives influence play on older machines. This would be a particularly important limitation if there was something distinctive about areas where the older machines are found. It is possible for example, low socio economic areas, which are associated with problem gambling (Sapere Research Group, 2018), are areas slower to update machines. If this was the case, we might not have been able to capture some key information about how gambling play occurs in key areas where harm minimisation tools are needed the most.

The current study used real world gaming information which differed from how this research is generally conducted in other studies (Gainsbury et al., 2015). Most studies within this literature field investigated the utility of pop-ups using data gathered from gambling simulations in laboratory settings. These types of studies allow careful control of variables and can isolate specific effects associated with particular variables. These type of studies do have a weakness in that players are not measured within a real gambling lounge setting, do not spend their own money and are often aware they are being observed (Jardin \& Wulfert, 2009, 2012; Monaghan, Blaszczynski, \& Nower, 2009). This can be problematic when drawing conclusions about how people will gamble in a natural gambling setting, with their own money. These two types of research tend to complement each other by being able to answer slightly different questions. The advantage of this study was that the data were collected from natural gambling sessions across the whole of New Zealand where the participants were not aware sessions were observed. However, the data were collected for regulatory functions and this reduced the control we had over what data were available and thus the variables we could investigate. However, we are confident the results reported are reflective of what occurred in the real world. 


\section{Summary}

Overall this study aimed to determine how effective both EGM pop-up displays and elective displays in New Zealand are. A dataset using real data from EGMs across New Zealand was used. The overall conclusion of the study was that neither type of information display had the intended harm minimisation effect. The data also provided evidence that variables such as venue type and manufacturer influenced the way in which both information displays are used. While these effects were small, it is likely effectiveness of information displays are influenced by other variables not tested in the current study. Through the findings of this study and the previous literature there are many suggested improvements that could be attempted to increase the utility of these harm minimisation tools. 


\section{References}

Abbott, M., Bellringer, M., \& Garrett, N. (2018). New Zealand National Gambling Study: Wave 4 (2015). Report Number 6. Auckland: Auckland University of Technology, Gambling and Addictions Research Centre.

Argo, J. J., \& Main, K. J. (2004). Meta-analyses of the effectiveness of warning labels. Journal of Public Policy and Marketing, 23(2), 193-208. doi:https://doi.org/10.1509/jppm.23.2.193.51400

Auer, M., \& Griffiths, M. D. (2013). Voluntary limit setting and player choice in most intense online gamblers: An empirical study of gambling behaviour. Journal of Gambling Studies, 29(4), 647-660.

Auer, M., \& Griffiths, M. D. (2015). Testing normative and self-appraisal feedback in an online slot-machine pop-up in a real-world setting. Frontiers in Psychology, 6, 339. doi:https://doi.org/10.3389/fpsyg.2015.00339

Auer, M., \& Griffiths, M. D. (2017). Self-reported losses versus actual losses in online gambling: An empirical study. Journal of gambling studies, 33(3), 795-806.

Auer, M., Hopfgartner, N., \& Griffiths, M. (2018). The effect of loss-limit reminders on gambling behavior: A real-world study of Norwegian gamblers. Journal of Behavioral Addictions, 7(4), 1056-1067. doi:https://doi.org/10.1556/2006.7.2018.106

Auer, M., Malischnig, D., \& Griffiths, M. (2014). Is" pop-up" messaging in online slot machine gambling effective as a responsible gambling strategy? Journal of Gambling Issues(29), 1-10. doi:https://doi.org/10.4309/jgi.2014.29.3

Benhsain, K., Taillefer, A., \& Ladouceur, R. (2004). Awareness of independence of events and erroneous perceptions while gambling. Addictive Behaviors, 29(2), 399-404. doi:https://doi.org/10.1016/j.addbeh.2003.08.011 
Binde, P., Romild, U., \& Volberg, R. A. (2017). Forms of gambling, gambling involvement and problem gambling: evidence from a Swedish population survey. International Gambling Studies, 17(3), 490-507. doi:https://doi.org/10.1080/14459795.2017.1360928

Blaszczynski, A., Cowley, E., Anthony, C., \& Hinsley, K. (2016). Breaks in play: Do they achieve intended aims? Journal of Gambling Studies, 32(2), 789-800. doi:https://doi.org/10.1007/s10899-015-9565-7

Blaszczynski, A., \& Nower, L. (2002). A pathways model of problem and pathological gambling. Addiction, 97(5), 487-499. doi:https://doi.org/10.1046/j.1360$\underline{0443.2002 .00015 . x}$

Breen, R. B., \& Zuckerman, M. (1999). Chasing'in gambling behavior: Personality and cognitive determinants. Personality and Individual Differences, 27(6), 10971111. doi:https://doi.org/10.1016/S0191-8869(99)00052-5

Cabinet Policy Committee. (2003). Gambling Act - Proposed Regulations (Harm Minimisation). New Zealand Retrieved from https://www.dia.govt.nz/diawebsite.nsf/Files/HarmMinimisationSDC0480/\$file/ HarmMinimisationSDC0480.pdf.

Calado, F., \& Griffiths, M. D. (2016). Problem gambling worldwide: An update and systematic review of empirical research (2000-2015). Journal of Behavioral Addictions, 5(4), 592-613. doi:https://doi.org/10.1556/2006.5.2016.073

Campbell-Meiklejohn, D. K., Woolrich, M. W., Passingham, R. E., \& Rogers, R. D. (2008). Knowing when to stop: The brain mechanisms of chasing losses. Biological Psychiatry, 63(3), 293-300. doi:https://doi.org/10.1016/j.biopsych.2007.05.014 
Chua, H. F., Ho, S. S., Jasinska, A. J., Polk, T. A., Welsh, R. C., Liberzon, I., \& Strecher, V. J. (2011). Self-related neural response to tailored smoking-cessation messages predicts quitting. Nature Neuroscience, 14(4), 426-427. doi:https://doi.org/10.1038/nn.2761

Cloutier, M., Ladouceur, R., \& Sévigny, S. (2006). Responsible gambling tools: Pop-up messages and pauses on video lottery terminals. The Journal of Psychology, 140(5), 434-438. doi:https://doi.org/10.3200/JRLP.140.5.434-438

Delfabbro, P. H., \& Winefeld, A. H. (2000). Predictors of irrational thinking in regular slot machine gamblers. The Journal of Psychology, 134(2), 117-128. doi:https://doi.org/10.1080/00223980009600854

Department of Internal Affairs. (2001). People's Participation in and Attitudes to Gaming, 1985-2000 Final Results of the 2000 Survey. Retrieved from Wellington:

https://www.dia.govt.nz/Pubforms.nsf/URL/PublicAttitudestowardsGamingpt1. pdf/\$file/PublicAttitudestowardsGamingpt1.pdf

Department of Internal Affairs. (2005). People's Participation in, and Attitudes to, Gambling, 1985-2005 Results of the 2005 survey. Retrieved from Wellington: https://www.dia.govt.nz/Pubforms.nsf/URL/GamblingParticipationSurvey19852005.pdf/\$file/GamblingParticipationSurvey1985-2005.pdf

Department of Internal Affairs. (2013). Gambling Fact Sheet \#1 Gambling Regulation in New Zealand Retrieved from https://www.dia.govt.nz/diawebsite.nsf/Files/GamblingFactSheetsFeb2013/\$file/FactSheet1-Feb2013.pdf

Department of Internal Affairs. (2016). New Zealand Class 4 Gaming Machine Appendix to the Australian/New Zealand Gaming Machine National Standard 
WHAT INFLUENCES NEW ZEALANDERS' INFORMATION DISPLAY USE? 139

2016. Retrieved from https://www.dia.govt.nz/diawebsite.nsf/Files/Australian-

$\underline{\text { New\%20Zealand-Gaming-Machine-National-Standard/\$file/NZ-Class-4- }}$

Gaming-Machine-Appendix-to-the-Australian-New\%20Zealand-Gaming-

Machine-National-Standard-2016.pdf.

Department of Internal Affairs. (2017). Gambling operators' key financial statistics

(actual) [Excel file]. Retrieved from:

https://www.dia.govt.nz/diawebsite.nsf/Files/Gambling-Statistics/\$file/2010-

$\underline{\text { 2017-Gambling-Expenditure-Statistics-a.xlsx }}$

Department of Internal Affairs. (2018). Quarterly Summary of Gaming Machines.

Retrieved from: https://www.dia.govt.nz/diawebsite.nsf/Files/Gambling-

$\underline{\text { Statistics-May-2018/\$file/Quarterly-Summary-of-GM-March-2018.pdf }}$

Dowling, N., Smith, D., \& Thomas, T. (2005). Electronic gaming machines: Are they the 'crack-cocaine' of gambling? Addiction, 100(1), 33-45.

doi:https://doi.org/10.1111/j.1360-0443.2005.00962.x

Echeburúa, E., Báez, C., \& Fernández-Montalvo, J. (1996). Comparative effectiveness of three therapeutic modalities in the psychological treatment of pathological gambling: Long-term outcome. Behavioural and Cognitive Psychotherapy, 24(1), 51-72. doi:https://doi.org/10.1017/S1352465800016830

Engs, R. C. (1989). Do warning labels on alcoholic beverages deter alcohol abuse? Journal of School Health, 59(3), 116-118. doi:https://doi.org/10.1111/j.1746$\underline{1561.1989 . t b 04676 . x}$

Fitzsimons, G. J., \& Lehmann, D. R. (2004). Reactance to recommendations: When unsolicited advice yields contrary responses. Marketing Science, 23(1), 82-94. doi:https://doi.org/10.1287/mksc.1030.0033 
Gainsbury, S. M., Abarbanel, B. L., Philander, K. S., \& Butler, J. V. (2018). Strategies to customize responsible gambling messages: A review and focus group study. BMC Public Health, 18(1), 1381. doi:https://doi.org/10.1186/s12889-018-6281$\underline{0}$

Gainsbury, S. M., Aro, D., Ball, D., Tobar, C., \& Russell, A. (2015). Optimal content for warning messages to enhance consumer decision making and reduce problem gambling. Journal of Business Research, 68(10), 2093-2101. doi:https://doi.org/10.1016/j.jbusres.2015.03.007

Gainsbury, S. M., \& Blaszczynski, A. (2012). Harm minimisation in gambling. In P. R \& R. D (Eds.), Harm Reduction in Substance use and High-Risk Behaviour: International Policy and Practice (pp. 263-278). Oxford, UK: Wiley- Blackwell.

Ginley, M. K., Whelan, J. P., Pfund, R. A., Peter, S. C., \& Meyers, A. W. (2017). Warning messages for electronic gambling machines: Evidence for regulatory policies. Addiction Research and Theory, 25(6), 495-504. doi:https://doi.org/10.1080/16066359.2017.1321740

Goodstadt, M. S., \& Caleekal-John, A. (1984). Alcohol education programs for university students: A review of their effectiveness. International Journal of the Addictions, 19(7), 721-741. doi:https://doi.org/10.3109/10826088409057219

Gray, R. (2011). New Zealanders' participation in gambling: Results from the 2010 Health and Lifestyles Survey: Health Sponsorship Council Wellington.

Griffiths, M. D. (1993). Fruit machine gambling: The importance of structural characteristics. Journal of Gambling Studies, 9(2), 101-120. doi:https://doi.org/10.1007/BF01014863 
Griffiths, M. D. (1994). The role of cognitive bias and skill in fruit machine gambling. British Journal of Psychology, 85(3), 351-369. doi:https://doi.org/10.1111/j.2044-8295.1994.tb02529.x

Hammond, D. (2011). Health warning messages on tobacco products: a review. Tobacco Control, 20(5), 327-337. doi:https://doi.org/10.1136/tc.2010.037630

Harris, A., \& Griffiths, M. D. (2017). A critical review of the harm-minimisation tools available for electronic gambling. Journal of Gambling Studies, 33(1), 187-221. doi:https://doi.org/10.1007/s10899-016-9624-8

Harris, A., \& Griffiths, M. D. (2018). The impact of speed of play in gambling on psychological and behavioural factors: A critical review. Journal of Gambling Studies, 34(2), 393-412. doi:https://doi.org/10.1007/s10899-017-9701-7

Hollingshead, S. J., Wohl, M. J., \& Santesso, D. (2019). Including personalized behavioral feedback in pop-up messages does not enhance limit adherence among gamblers. Computers in Human Behavior, 94, 122-130. doi:https://doi.org/10.1016/j.chb.2019.01.015

Jacobs, D. F. (1988). Evidence for a common dissociative-like reaction among addicts. Journal of Gambling Behavior, 4(1), 27-37. doi:https://doi.org/10.1007/BF01043526

Jardin, B., \& Wulfert, E. (2009). The use of messages in altering risky gambling behavior in college students: An experimental analogue study. The American Journal on Addictions, 18(3), 243-247. doi:https://doi.org/10.1080/10550490902786918

Jardin, B., \& Wulfert, E. (2012). The use of messages in altering risky gambling behavior in experienced gamblers. Psychology of Addictive Behaviors, 26(1), 166-170. doi:https://doi.org/10.1037/a0026202 
Johnston, W. A., \& Dark, V. J. (1986). Selective attention. Annual Review of Psychology, 37(1), 43-75. doi:https://doi.org/10.1146/annurev.ps.37.020186.000355

Kairouz, S., Paradis, C., \& Monson, E. (2015). Does context matter? A multilevel analysis of gambling settings among undergraduates. Addiction Research \& Theory, 23(6), 518-527. doi:https://doi.org/10.3109/16066359.2015.1059826

Kim, H. S., Wohl, M. J., Stewart, M. J., Sztainert, T., \& Gainsbury, S. M. (2014). Limit your time, gamble responsibly: Setting a time limit (via pop-up message) on an electronic gaming machine reduces time on device. International Gambling Studies, 14(2), 266-278. doi:https://doi.org/10.1080/14459795.2014.910244

King, G., \& Zeng, L. (2001a). Explaining rare events in international relations. International Organization, 55(3), 693-715.

King, G., \& Zeng, L. (2001b). Logistic regression in rare events data. Political Analysis, 9(2), 137-163. doi:https://doi.org/10.1093/oxfordjournals.pan.a004868

Ladouceur, R., \& Sevigny, S. (2003). Interactive messages on video lottery terminals and persistence in gambling. Gambling Research: Journal of the National Association for Gambling Studies (Australia), 15(1), 44-49.

Ladouceur, R., \& Sévigny, S. (2009). Electronic gambling machines: Influence of a clock, a cash display, and a precommitment on gambling time. Journal of Gambling Issues(23), 31-41. doi:https://doi.org/10.4309/jgi.2009.23.2

Landon, J., Palmer du Preez, K., Bellringer, M., Page, A., \& Abbott, M. (2016). Pop-up messages on electronic gaming machines in New Zealand: experiences and views of gamblers and venue staff. International Gambling Studies, 16(1), 4966. doi:10.1080/14459795.2015.1093535 
WHAT INFLUENCES NEW ZEALANDERS' INFORMATION DISPLAY USE? 143

Landon, J., Thorne, H., Palmer, K., Page, A., \& Abbott, M. (2010). Focused Literature Review for the Problem Gambling Programme. Auckland: Auckland Univeristy of Technology, Gambling and Addictions Research Centre.

Leino, T., Torsheim, T., Pallesen, S., Blaszczynski, A., Sagoe, D., \& Molde, H. (2016). An empirical real-world study of losses disguised as wins in electronic gaming machines. International Gambling Studies, 16(3), 470-480. doi:https://doi.org/10.1080/14459795.2016.1232433

Lin, E., Casswell, S., \& You, R. (2008). Assessment of the social impacts of gambling in New Zealand. Auckland: Centre for Social and Health Outcomes Research and Evaluation and Te Ropu Whariki.

Ministry of Health. (2010). Preventing and Minimising Gambling Harm: Six-year strategic plan 2010/11-2015/16. Retrieved from Wellington: Ministry of Health: https://www.health.govt.nz/publication/preventing-and-minimising-gambling$\underline{\text { harm-six-year-strategic-plan-2010-11-2015-16 }}$

Ministry of Health. (2018). Clients Assisted by Primary Problem Gambling Mode [Excel file]. Retrieved from: https://www.health.govt.nz/our-work/mentalhealth-and-addictions/gambling/service-user-data/intervention-client-data

Mishra, S., Morgan, M., Lalumiere, M. L., \& Williams, R. J. (2010). Mood and audience effects on video lottery terminal gambling. Journal of Gambling Studies, 26(3), 373-386.

Monaghan, S. (2008). Review of pop-up messages on electronic gaming machines as a proposed responsible gambling strategy. International Journal of Mental Health and Addiction, 6(2), 214-222. doi:https://doi.org/10.1007/s11469-007-9133-1

Monaghan, S. (2009). Responsible gambling strategies for Internet gambling: The theoretical and empirical base of using pop-up messages to encourage self- 
awareness. Computers in Human Behavior, 25(1), 202-207. doi:https://doi.org/10.1016/j.chb.2008.08.008

Monaghan, S., \& Blaszczynski, A. (2007). Recall of electronic gaming machine signs:

A static versus a dynamic mode of presentation. Journal of Gambling Issues(20), 253-267. doi:https://doi.org/10.4309/jgi.2007.20.8

Monaghan, S., \& Blaszczynski, A. (2009). Electronic gaming machine warning messages: Information versus self-evaluation. The Journal of Psychology, 144(1), 83-96. doi:https://doi.org/10.1080/00223980903356081

Monaghan, S., \& Blaszczynski, A. (2010). Impact of mode of display and message content of responsible gambling signs for electronic gaming machines on regular gamblers. Journal of Gambling Studies, 26(1), 67-88. doi:https://doi.org/10.1007/s10899-009-9150-Z

Monaghan, S., Blaszczynski, A., \& Nower, L. (2009). Do warning signs on electronic gaming machines influence irrational cognitions? Psychological Reports, 105(1), 173-187. doi:https://doi.org/10.2466/PR0.105.1.173-187

Palmer du Preez, K., Landon, J., Bellringer, M., Garrett, N., \& Abbott, M. (2016). The effects of pop-up harm minimisation messages on electronic gaming machine gambling behaviour in New Zealand. Journal of Gambling Studies, 32(4), 11151126. doi:https://doi.org/10.1007/s10899-016-9603-0

Palmer du Preez, K., Landon, J., Garrett, N., Bellringer, M., Page, A., Coomarasamy, C., \& Abbott, M. (2014). Investigation into the effects of gambling game characteristics, PIDS and pop-up technology on gambling and problem gambling behaviour in New Zealand. Auckland: Auckland University of Technology, Gambling and Addictions Research Centre.

Productivity Commission. (2001). Australia's gambling industries. Retrieved from 
WHAT INFLUENCES NEW ZEALANDERS' INFORMATION DISPLAY USE? 145

Productivity Commission. (2010). Annual Report 2009-10. Retrieved from Productivity Commission, Canberra: https://www.pc.gov.au/about/governance/annualreports/annualreport0910/annual-report-2009-10.pdf

Robinson, T. N., \& Killen, J. D. (1997). Do cigarette warning labels reduce smoking?: paradoxical effects among adolescents. Archives of pediatrics \& adolescent medicine, 151(3), 267-272.

Sagoe, D., Pallesen, S., Griffiths, M. D., Mentzoni, R. A., \& Leino, T. (2018). Does Individual Gambling Behavior Vary across Gambling Venues with Differing Numbers of Terminals? An Empirical Real-World Study using Player Account Data. Frontiers in Psychology, 9, 158.

doi:https://doi.org/10.3389/fpsyg.2018.00158

Sapere Research Group. (2018). Gambling Harm Reduction Needs Assessment. Retrieved from Wellington: Ministry of Health:

https://www.health.govt.nz/publication/gambling-harm-reduction-needs$\underline{\text { assessment }}$

Schellink, T., \& Schrans, T. (2002). Atlantic Lottery Corporation video lottery responsible gaming feature research. Halifax, Nova Scotia: Focal Research Consultants.

Sharpe, L., Walker, M., Coughlan, M.-J., Enersen, K., \& Blaszczynski, A. (2005). Structural changes to electronic gaming machines as effective harm minimization strategies for non-problem and problem gamblers. Journal of Gambling Studies, 21(4), 503-520. doi:https://doi.org/10.1007/s10899-005$\underline{5560-8}$

Smith, G. J., \& Wynne, H. J. (2004). VLT gambling in Alberta: A preliminary analysis. Retrieved from 
WHAT INFLUENCES NEW ZEALANDERS’ INFORMATION DISPLAY USE? 146

https://prism.ucalgary.ca/bitstream/handle/1880/1632/VLT_Gambling_Alberta.p $\underline{\text { df? }}$ sequence $=1 \&$ isAllowed $=y$

Steenbergh, T. A., Whelan, J. P., Meyers, A. W., May, R. K., \& Floyd, K. (2004). Impact of warning and brief intervention messages on knowledge of gambling risk, irrational beliefs and behaviour. International Gambling Studies, 4(1), 316. doi:https://doi.org/10.1080/1445979042000224377

Stewart, M. J., \& Wohl, M. J. (2013). Pop-up messages, dissociation, and craving: How monetary limit reminders facilitate adherence in a session of slot machine gambling. Psychology of Addictive Behaviors, 27(1), 268.

doi:https://doi.org/10.1037/a0029882

Stockley, C. S. (2001). The effectiveness of strategies such as health warning labels to reduce alcohol-related harms — an Australian perspective. International Journal of Drug Policy, 12(2), 153-166. doi:https://doi.org/10.1016/S0955$\underline{3959(01) 00077-9}$

Sylvain, C., Ladouceur, R., \& Boisvert, J.-M. (1997). Cognitive and behavioral treatment of pathological gambling: A controlled study. Journal of Consulting and Clinical Psychology, 65(5), 727-732. doi:https://doi.org/10.1037/0022$\underline{006 X .65 .5 .727}$

Tanner, J., Drawson, A. S., Mushquash, C. J., Mushquash, A. R., \& Mazmanian, D. (2017). Harm reduction in gambling: A systematic review of industry strategies. Addiction Research and Theory, 25(6), 485-494. doi:https://doi.org/10.1080/16066359.2017.1310204

Taylor, L. F., Macaskill, A. C., \& Hunt, M. J. (2017). Realistic free-spins features increase preference for slot machines. Journal of Gambling Studies, 33(2), 555577. doi:https://doi.org/10.1007/s10899-016-9630-x 
Thomas, J., Mora, K., \& Rive, G. (2010). An investigation of the influence of gambling venue characteristics on gambler's behaviour. Retrieved from Lower Hutt: Opus International Consultants Limited https://www.health.govt.nz/system/files/documents/publications/influencegambling-venue-characteristics.doc

True, J., \& Cheer, M. (2018). Gaming Machine Gambling Statistics and Research Paper - Information for Territorial Authorities. Retrieved from http://www.gamblinglaw.co.nz/download/Research/TAInfo.pdf

Turner, N., \& Horbay, R. (2004). How do slot machines and other electronic gambling machines actually work? Journal of Gambling Issues(11). doi:https://doi.org/10.4309/jgi.2004.11.21

Van Swol, L. M., MacGeorge, E. L., \& Prahl, A. (2017). Advise with permission?: The effects of advice solicitation on advice outcomes. Communication Studies, 68(4), 476-492. doi:https://doi.org/10.1080/10510974.2017.1363795

Wohl, M. J., Gainsbury, S., Stewart, M. J., \& Sztainert, T. (2013). Facilitating responsible gambling: The relative effectiveness of education-based animation and monetary limit setting pop-up messages among electronic gaming machine players. Journal of Gambling Studies, 29(4), 703-717. doi:https://doi.org/10.1007/s10899-012-9340-y

Wood, R. T., \& Wohl, M. J. (2015). Assessing the effectiveness of a responsible gambling behavioural feedback tool for reducing the gambling expenditure of atrisk players. International Gambling Studies, 15(2), 1-16. doi:https://doi.org/10.1080/14459795.2015.1049191

Wynne, H. J. (2002). Gambling and problem gambling in Saskatchewan. Retrieved from 
Wynne, H. J., \& Stinchfield, R. (2004). Evaluating VLT responsible gaming features and interventions in Alberta: Phase I-final report. Retrieved from https://prism.ucalgary.ca/bitstream/handle/1880/48211/VLT_responsible_featur es_phase1_report.pdf?sequence $=1 \&$ is Allowed $=y$ 


\section{Appendix A}

Shannon Garland Duignan: Thesis Study One proposal

Summary: I propose to request and analyse a dataset of EMG PID session stopped and elective information display button events in order to better understand the impact of these machine features.

For my Masters thesis I am interested in the topic of pokie harm minimisation in New Zealand. A particular topic of interest is interruptive information displays that are required as part of the Gaming Machine National Standard Revision 10.0 published 1 October 2009. The first particular question that we want to answer as part of my thesis is how these function in practice.

This project will build on a study completed by Auckland University of Technology which also looked into the effect of pop-up displays on pokie machine gambling. Their study used focus groups and surveys to assess the effect of pop-ups leading to the conclusion that for some people they are an effective way to control gambling. Our study will use a quantitative approach to help understand why they aren't effective all the time. This will allow us to develop a better understanding of how player information displays function in New Zealand.

The Electronic Monitoring System (EMS), used by DIA as part of the regulatory tools, records a large number of events and meter information regarding play on gaming machines across the whole of New Zealand. We wish to utilise the data stored on the EMS database, collected from real New Zealand venues and analyse it to answer questions regarding the usefulness of the popup displays.

The minimum technical requirements for the electronic monitoring system for gaming machines (that run on the QCOM version 1.6.3 technology), specify certain events that must be recorded. The event of particular interest to us is called "EGM PID (player information display) session stopped", which notifies the end of a person's gambling session on a particular machine. A session begins when a player places a bet and ends when a) a player does not play a game and a period of 60 seconds has elapsed since the end of the last completed game; or b) no further play is possible without additional credits being purchased by the player and 30 seconds has elapsed since the last completed game; or c) a player has exited the game by pressing the collect button.

The information that is captured in this "EMG PID session stopped" event includes:

- The duration of the session;

- The number of Interruptive information displays that have been displayed during the session;

- The amount the player spent in the session;

- Their net win or loss at the time the session ended;

- How the session was stopped - whether the player stopped the session by clicking 'no' button in response to being asked if they wish to continue play on a pop-up screen, or whether they stopped at any other time.

We understand that there is a large amount of data available so we will need to discuss with the appropriate people as to how much data is practically possible to extract and analyse. We are interested in gathering as much data as possible so that we can first use part of the data explore possible trends present and then use the rest of the data to confirm which of these trends are reliable.

To assess the usefulness of pop-up player information displays we want to first look at how many people exit a game at a pop-up display. This will give us an initial indication of the extent 
to which the displays influence gambling. If this number is low then a follow up study could look at why the pop-ups are not aiding people to stop gambling, which is what they are designed to do.

If there are enough sessions on which players exit the session via the pop-up we will run an analysis comparing those who stop at a pop-up, compared to those that stop at any other time. We propose to firstly look at how session duration, player spending, and net win/loss for a session differ, based on the situation in which they exit the game. As the time that a person has been playing for is correlated with the amount of money that a person spends, we would calculate money spent per minute, which is a measure that would be comparable across different sessions. We would then run a statistical analysis allowing us to see whether these factors significantly predict their session end type (pop-up or not at a pop-up). This would allow us to see under what circumstances the pop-up information displays are most effective as a tool to assist people exiting out of playing.

Answering this question would hopefully identify particular features or sets of features about play that lead people to be more responsive to pop-ups. This could for example be when the player is currently winning, or could be after a certain amount of time has passed, or after they have seen a few pop-up displays.

These types of findings would be useful for more effective targeting of pop-up displays and could also inform what messaging may be useful to use in such displays. For example, if time spent on a machine seems to be a predictor, then this could help inform wording that may be useful such saying 'how long have you been playing, is it time to stop?'. The pop-up could highlight the time that they have been playing for, such as "you have been playing for XX hours, what else could you be using this time for?".

Machines also record when people access the voluntary player information display which contains information about the odds of winning various games. We also want to analyse the data associated with this feature as it also functions as a harm minimisation tool. Initially we would want to look at whether these are used a sufficient number of times to plausibly have a harm minimisation effect.

This research will ultimately be available in the university library as part of my thesis, I will present it at academic conferences, and it will be submitted for possible publication in academic journals. We will never include any information that might specifically identify a venue when presenting this research in any form.

This is still in the planning phase so please feel free to comment on anything that could be improved on or any related ideas that might be interesting to explore next. Please contact me via email: garlanshan@myvuw.ac.nz. 


\section{Appendix B}

\section{Data Sharing and Use Agreement}

to facilitate analysis required to complete a thesis

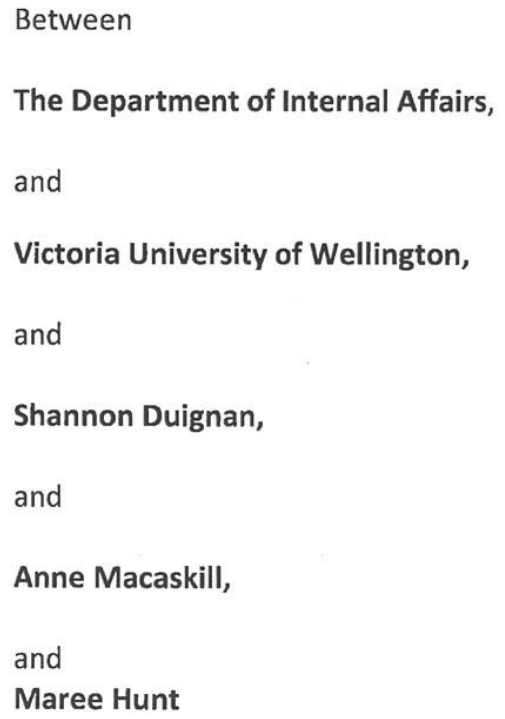




\section{Data Sharing and Use Agreement}

to facilitate analysis required to complete a thesis

Parties:

The parties to this data sharing and use agreement are:

A. The Sovereign in right of New Zealand acting by and through the Chief Executive of the Department of Internal Affairs (DIA);

B. Victoria University of Wellington (VUW);

C. Shannon Garland Duignan (the Author);

and

D. Anne Macaskill; and

E. Maree Hunt,

(together the Supervisors).

1) Background:

a) The Author intends to research, prepare, submit, and ultimately publish the Thesis as part of her programme of study at Victoria University. The Author and Supervisors intend to present the results of this project at academic conferences, and in academic journals.

b) The Supervisors will supervise the Author's work.

c) DIA holds the Data as part of its role in enforcing and administering the Act.

d) DIA agrees to share the Data with VUW, the Author, and the Supervisors, and those parties agree to receive and use the Data, in accordance with the terms of this Agreement.

e) In consideration for DIA's support and willingness to share the Data, the Author grants DIA the rights set out in this Agreement in relation to the Thesis.

2) Interpretation

a) Capitalised terms in this Agreement will have the meaning set out below:

i) Accession Deed means the deed entered into by an Acceding Party substantially in the form attached as Schedule 1, whereby that Acceding Party agrees to be bound by the same obligations as the Supervisor for the Term; 
ii) Acceding Party has the meaning given in clause $4(\mathrm{a})(\mathrm{i})$;

iii) Act means the Gambling Act 2003;

iv) Agency means a New Zealand or overseas law enforcement agency, or other agency, that regulates, monitors or researches Gambling Activities or related activities, Agencies has a corresponding meaning;

v) Agreement means this data sharing and use agreement, and includes any Schedules;

vi) Business Day means Monday to Friday excluding any public holidays observed in Wellington;

vii) Business Hours means 9am to $5 \mathrm{pm}$ on a Business Day;

viii) Commencement Date has the meaning given in 5(a);

ix) Contact Person means Bruce Manuge, Senior Technical Advisor, Gambling Technology, or Michelle White, Manager Sector Initiatives or another DIA staff member advised by DIA replacing one or both these people, and Contact People has a corresponding meaning;

x) Data means the information relating to Machine usage, operation and other related matters, recorded and held in the Database;

xi) Database means the system or systems within which DIA stores the Data, and from which DIA retrieves the Data;

xii) Flash Drive means a password protected flash drive provided to the Author by DIA ;

xiii) Final Draft means the draft version of the Thesis that is a substantively final version to be submitted to Victoria University for publishing;

xiv) Gambling Activity means activity utilising, or relating to, a Machine;

xv) Individual means an identifiable person who is engaged or involved in Gambling Activity, including people gamblers, and those providing or servicing Machines at Venues;

xvi) Initial Request means the first request the Author makes for the Data following the Commencement Date;

xvii) Initial Request Date means the date the Author makes the Initial Request during the Term;

xviii) Machine means a gaming machine, as that term is defined in the Act; 
xix) Published Version means the version of the Thesis that is published and available as a final version through the Victoria University library, and that is then available for presentation at conferences and publication in academic journals in whole, part, or modified form;

xx) Schedule means a schedule attached to this Agreement;

xxi) Service Agreement means any agreement between DIA and its service providers in relation to the systems that support the Database, the Machines, and the Department's regulatory role in relation to Gambling Activities;

xxii) Summarised Data means totals, means, medians, and other statistics calculated from the Data but not including individual records of specific gambling sessions;

xxiii) System has the meaning given in clause 3(b)(ii);

xxiv) Term has the meaning given in clause 5(a) of this Agreement;

xxv) Thesis means the body of work produced by the Author as part of her study;

xxvi) Venue means those premises in which Machines are located, and utilised, for gambling; and

xxvii) Victoria University means the Victoria University of Wellington.

\section{3) Agreement}

a) DIA agrees:

i) to physically provide the Data to the Author on the Flash Drive as soon as possible on or after the Initial Request Date, such date to be determined in the Author's sole discretion;

ii) to provide any other Data after the Initial Request Date as requested and agreed between the parties, to the Author in sensible batches on a Flash Drive;

iii) to retain the Data in the Database, or on another secure system or systems from which it can be retrieved, for the Term;

iv) the Data will not include the name, or any other identifying information about, any Individual;

v) to make the Contact Person available for the Author to phone, visit, or email during Business Hours to inquire about the Data;

vi) to grant VUW, the Author and the Supervisors all the rights they need in order to make use of, reference, and share, the Data in accordance with this Agreement; 
WHAT INFLUENCES NEW ZEALANDERS' INFORMATION DISPLAY USE? 155

vii) to only share the Final Draft with such DIA staff as is sensible and required in order for DIA to get the full benefit from the Final Draft in relation to DIA's regulatory and other activities;

viii) to provide all comments to the Author on the Final Draft (once received) in a timely manner, acknowledging that the Author is under no obligation to incorporate such comments unless they relate to maintenance or compliance with the law;

ix) to only share the Final Draft with any third party following receipt of prior written consent from the Author;

x) to not make unnecessary copies of the Final Draft;

xi) to destroy all copies of the Final Draft in its possession once the searchable Published Thesis has been supplied to DIA by the Author, and to request that all third parties who have been provided with a Final Draft by DIA, also destroy their copy or copies;

xii) to not directly quote the Final Draft in any material released to any third party outside DIA without the Author's prior approval; and to reference any authorised direct quote, or summary of, the findings of the Final Draft in such material, by using the citation details provided by the Author;

xiii) to always cite the Published Thesis when quoting from it, or referencing it, using the citation details provided by the Author; and

xiv) that, except for the rights granted in this Agreement, the Crown has no ownership interest in the Final Draft, the Published Thesis, nor any other material produced by the Author or the Supervisors; and

xv) to ensure that any third party who receives a copy of the Final Draft from DIA first agrees to: use it in accordance with the terms set out above; not disclose it to any other party, and agrees to destroy it when requested by DIA.

b) VUW agrees:

i) to procure that each Hard-drive is accessible only to the Author, and the Supervisors (as relevant) during the Term, provided that each Hard-drive will be backed up onto secure, university servers (System);

ii) to execute any confidentiality undertaking or other assurance that the Department is required to procure under any Service Agreement;

iii) to not electronically transfer the Data in any way out of the System to any third party, or upload the Data onto any cloud based Internet tool outside the System, without DIA's express written approval;

iv) to not make unnecessary copies of the Data; 
WHAT INFLUENCES NEW ZEALANDERS’ INFORMATION DISPLAY USE? 156

v) to only use the Data for research and teaching related matters, including producing journal articles and conference presentations, and for no other purpose.

vi) to procure DIA is supplied with a searchable electronic copy of the Final Draft as soon as it is available (including any substantive update of the Final Draft), along with full instructions on how to cite the Final Draft;

vii) to procure that the Thesis is not published, until any comments made by DIA have been considered and any DIA concerns about: the privacy of Individuals; identification of Venue related or other information; or maintenance of, and compliance with, the law, have been resolved to DIA's satisfaction;

viii) to procure DIA is supplied with a searchable electronic copy of the Published Thesis as soon as possible, along with full instructions on how to cite the Published Thesis;

ix) that DIA may make copies of the Published Thesis and share those copies with Agencies, without requiring the consent of VUW;

x) to grant DIA all the rights it needs in order to make use of, reference and share, the Final Draft and the Published Thesis as described in this Agreement;

xi) to take whatever action can be taken to prevent or mitigate any loss, risk or damage resulting from unauthorised access to the Data, and notify DIA immediately, should it become aware that any breach of these terms has occurred, or is suspected;

xii) to comply with all DIA's instructions in relation to the Data, including any direction to destroy the copy of the Data held in the System or elsewhere, for any reason, including in response to a privacy complaint or a reasonable concern about security or release of commercially sensitive information;

xiii) to acknowledge that the Data was created for internal DIA use, and is intended for viewing by a specialist internal audience, therefore such Data is received by the Author on an "as is" basis, and DIA makes no warranty as to:

(1) the accuracy or consistency of the Data; or

(2) the completeness of the Data; and

xiv) that, except for the rights granted in this Agreement, VUW has no ownership interest, nor any other interest, in the Data.

c) The Author agrees:

i) to ensure the Initial Request is sent by email to the Contact Person, and to follow up any other verbal requests for Data with an email to the Contact Person; 
ii) to download all Data immediately on receipt of a Flash Drive from DIA directly onto her secure Victoria University computer hard-drive, and to procure anothe copy of the Data is downloaded onto the Supervisor's secure Victoria University computer hard-drive (each a Secure Hard Drive);

iii) to execute any confidentiality undertaking or other assurance that the Department is required to procure under any Service Agreement;

iv) to ensure the Flash Drive is returned to DIA, immediately following download of the Data onto the System;

v) to not electronically transfer the Data in any way out of the System to any third party, or upload the Data onto any cloud based Internet tool outside the System, without DIA's express written approval;

vi) to not make unnecessary copies of the Data, and to produce and keep all notes, records, drafts and other materials produced during the development of the Thesis referencing the Data in such a way as Individuals or Venues cannot be identified;

vii) to only use the Data for research, development and assessment of the Thesis, including for producing journal articles and conference presentations;.

viii) to ensure that the Thesis does not contain the name, address or any other identifying information about an Individual or Venue, and that the Data is only reported in the Thesis in an aggregated manner that would not allow a third party, reading the Thesis alongside publicly available material, to work out more information about an Individual or a Venue than would be discoverable from an careful analysis of publicly available information;

ix) that until a published version of the Thesis is available, DIA may use and share the Final Draft as set out in clause 3(a) above;

x) to respond in a timely manner to any request from DIA to share the Final Draft with a third party;

xi) that DIA may make copies of the Published Thesis and share those copies with Agencies, without requiring the consent of the Author or VUW;

xii) to grant DIA all the rights it needs in order to make use of, reference and share, the Final Draft and the Published Thesis as described in this Agreement;

xiii) to take whatever action can be taken to prevent or mitigate any loss, risk or damage resulting from unauthorised access to the Data, and notify DIA immediately, should she become aware that any breach of these terms has occurred, or is suspected;

xiv) to comply with all DIA's instructions in relation to the Data, including any direction to destroy the copy of the Data held in the System or elsewhere, for any reason, 
including in response to a privacy complaint or a reasonable concern about security or release of commercially sensitive information;

xv) to acknowledge that the Data was created for internal DIA use, and is intended for viewing by a specialist internal audience, therefore such Data is received by the Author on an "as is" basis, and DIA makes no warranty as to:

(1) the accuracy or consistency of the Data; or

(2) the completeness of the Data; and

xvi) that, except for the rights granted in this Agreement, the Author has no ownership interest, nor any other interest, in the Data.

d) Each Supervisor agrees:

i) to be bound by the obligations set out in clauses 3(c)(iii), (v)-(vii) and (xiii)-(xvi) as if she was the Author, with the necessary modifications.

4) Acceding Parties

a) The parties agree that:

i) the Author or VUW will advise DIA immediately should any third party (such as anyone who becomes part of a research team for production of the Thesis) require access to any part, or all, of the Data (Acceding Party);

ii) the Author or VUW will procure that:

(1) the Acceding Party has received and understood the terms of this Agreement and the obligations of each Supervisor under this Agreement, prior to entering the Accession Deed; and

(2) DIA is provided with an executed copy of the Accession Deed entered into by any Acceding Party, prior to that Acceding Party viewing any Data; and

iii) the same obligations will apply in relation to any Acceding Party as to each Supervisor, and each reference to a Supervisor will be read as the Acceding Party in relation to that party's obligations (with necessary amendments), effective from the earlier of:

(1) the date the Acceding Party enters the Accession Deed and becomes a party to this Agreement; or

(2) the Acceding Party views the Data.

5) Term

a) This Agreement will commence on the date the last party executes the Agreement (Commencement Date), and will end on the date that is the earlier of: 
i) the date the Agreement is terminated in accordance with clause 5(b); and

ii) 31 December 2020 .

(Term)

b) The Agreement will terminate immediately if:

i) DIA gives written notice to the Author that all the Data must be destroyed in accordance with this Agreement;

ii) the Author gives notice, or it becomes apparent to DIA, that she cannot, or will not, finish the Thesis;

iii) the Author does not make the Initial Request within the Term; or

iv) The Author gives notice, or it becomes apparent to DIA, that the Final Draft will never be published for whatever reason.

c) On expiry or termination of the Agreement:

i) VUW, the Author and each Supervisor will immediately destroy: all copies of the complete Data on the System, or in their possession, provided that:

(1) one copy of the complete Data may be kept on the System for access by the Supervisor for one year following submission of the Thesis for publication; and

(2) a copy of the Summarised Data may be kept indefinitely for the purpose of fulfilling journal publication requirements, provided that VUW may share this with other researchers at their request.

ii) at the end of the period referred to in the proviso in clause 5 (c)(i)(1), the Supervisor must immediately destroy the complete Data in full compliance with this clause $5(\mathrm{c})$;

iii) On request, VUW, the Author or a Supervisor (as relevant) will each provide DIA with written notice confirming that the actions described in clauses 5(c)(i) and 5(c)(ii) respectively have been taken, and that no party (including a Supervisor) can access the Data;

iv) clauses that are intended to survive expiry or termination will do so, including clauses 2, parts of 3 that are relevant for the period post termination or expiry, 4(a)(iii), 5(c), 6 and 7;

v) if this Agreement is terminated under clause $5(b)(i))$, and DIA has a copy of the Final Draft, DIA may continue to retain and use the Final Draft in accordance with this Agreement until the Author advises otherwise; and

vi) any Accession Deed will expire or terminate, and the Author will give notice of such expiry or termination to the Acceding Party. 


\section{6) Disputes and disagreements}

a) The parties will discuss and resolve all disputes and disagreements by agreement between the Author and the Contact Person.

b) Should a matter not be resolved under clause $6(a)$ it shall be referred to a senior staff member of Victoria University, and the DIA General Manager Regulatory Services (or equivalent) for resolution.

c) Nothing in this Agreement will preclude either party (including any Acceding Party) from taking immediate steps to seek urgent relief before a New Zealand Court.

7) General

(a) This Agreement may be amended at any time by a written Agreement between the parties, provided that any Acceding Party will be deemed to have agreed to be bound by the Agreement as varied and the Author will give notice of such amendment to each Acceding Party.

(b) Subject to any disclosure required by law or parliamentary convention, the terms of this Agreement will be kept confidential, and will not be disclosed to any person without the prior written consent of the Author and DIA (as relevant), except to those persons who need to have access to it in order to perform their role, and to any Acceding Party prior to entry into the Accession Deed.

(c) Any notice or other document to be given in connection with this Agreement is to be delivered by hand or sent by post or email to the relevant address or addresses stated below. Each Supervisor and all Acceding Parties will be deemed to have been served notice at the same time the Author receives notice from any other party:

DIA:

To: $\quad$ Maarten Quivooy, General Manager, Regulatory Services

Postal address: Department of Internal Affairs, PO Box 805, Wellington

Email: marten.quivooy@dia.govt.nz

Copy to: Chief Legal Adviso

Postal address: Department of Internal Affairs, PO Box 805, Wellington Email:_legal.notices@dia.govt.nz

VUW

Postal address: Dan Thompson, Contracts Manager, Research Development Office Rankine Brown 905

Victoria University of Wellington

PO Box 600

Wellington 6140

Email:

dan.thompson@vuw.ac.nz

Author: 
WHAT INFLUENCES NEW ZEALANDERS' INFORMATION DISPLAY USE? 161

Postal address: Shannon Duignan, C/- Dan Thompson, Contracts Manager, Research Development Office

Rankine Brown 905

Victoria University of Wellington

PO Box 600

Wellington 6140

Email: Shannon.garlandduignan@vuw.ac.nz

Notice sent by:

a. post, will be deemed to have been delivered on the third Business Day following posting;

b. email before $5 \mathrm{pm}$, will be deemed to have been delivered at the time the email leaves the communications system of the sender, provided the sender does not receive an error message relating to the sending of the email; and

c. email after $5 \mathrm{pm}$, will be deemed to have been delivered the next Business Day after the email leaves the communications system of the sender, provided the sender does not receive an error message relating to the sending of the email.

(d) This Agreement is governed by, and will be construed in accordance with, the laws of New Zealand. Each party submits to the exclusive jurisdiction of the New Zealand Courts in respect of any dispute or other matter arising under this Agreement.

This Agreement may be signed in counterparts each of which, when taken together, will constitute one and the same Agreement. A party may enter into this Agreement by signing any counterpart.

Executed

SIGNED for and on behalf of The Sovereign in Right of New Zealand acting by and through the Chief Executive of the

Department of Internal Affairs:

$$
\text { Date: } 26 / 5 / 18
$$

SIGNED for and on behalf of Victoria Unfverşity of Wellington:

Date: $30 / 05 / 2018$

Signed by Shannon Garland Duignan: 
Date: $30 / 5 / 18$

Signed by Anne Macaskill:

Date: $3015 / 18$

Signed by Maree Alunt:

Date: $30 / 5 / 18$ 


\section{Schedule 1}

\section{Accession Deed}

I [insert name] agree and acknowledge:

1) that I have received a copy of, and read and understood, the Data Sharing and Use Agreement between The Sovereign in Right of New Zealand acting by and through the Chief Executive of the Department of Internal Affairs (DIA), Shannon Duignan (Author), and Anne Macaskill and Maree Hunt (each a Supervisor), (the Agreement), prior to receiving access to any data or material received by the Author from DIA in relation to Gambling Activities;

2) effective from the date that I sign this Accession Deed, I am a party to the Agreement and subject to the same rights and obligations as a Supervisor (to the extent relevant) in relation to its subject matter;

3) the parties by vary or terminate the Agreement in accordance with its terms at any time without consulting me, and such variation or termination will be deemed to apply to me and this Accession Deed from its effective date;

4) to avoid doubt:

a) this Accession Deed will expire or terminate automatically on expiry or termination of the Agreement; and

b) the clauses intended to survive expiry or termination of the Agreement, will continue to apply to me as relevant.

SIGNED and delivered as a Deed by:

Signature

Full Name

Occupation

Town or city

Date: 